\title{
Temperature-Dependent Fracture Toughness Evaluation of WC-10Co4Cr Coating/1018 Low Carbon Steel Substrate System
}

by

\section{Yichen Gu}

\begin{abstract}
A thesis submitted to the Faculty of Graduate and Postdoctoral Affairs in partial fulfillment of the requirements for the degree of
\end{abstract}

Master of Applied Science

in

Mechanical Engineering

Carleton University

Ottawa, Ontario

(C)2015, Yichen Gu 


\section{Abstract}

The temperature-dependent fracture toughness of $\mathrm{WC}-10 \mathrm{Co} 4 \mathrm{Cr}$ coating/1018 low carbon steel substrate, which is a brittle coating/ductile substrate system, is evaluated at microscopic level with two types of models developed in terms of the Arrhenius-type equation and rate controlling theory, utilizing indentation test data, in this research. One is based on the crystal structures of individual phases in the composite coating, the other considers the microcrack formation in the coating/substrate system under indentation loading. Using the crystal-structure-based model, the slip systems of the hard hexagonal $\delta$-WC phase and soft FCC $\alpha$-Co phase are analyzed. The fracture toughness of the two-phase composite coating is obtained by integrating the fracture toughness of $\delta$-WC phase and $\alpha$-Co phase using either the basic mixture method or the unconstrained mixture method. The estimated fracture toughness using this type of model is independent of indentation load. For the microcrack-formation-based models, numerous microcracks are generated from each corner of indentation impression and merge together to form radial cracks due to the tension of residual stresses in the coating/substrate system under indentation, and the radial cracks extend along the indentation diagonals under the residual stresses. The dislocation movement of atoms can be associated with the microcrack formation in the indentation process thus the fracture toughness of the composite coating/substrate system is evaluated. Due to the effect of ductile substrate on brittle coating, two approaches are used to investigate the indentation pressure imposed on the system, one expresses the indentation pressure as the applied load 
divided by the projected area of indentation impression, the other is based on the composite hardness of the coating/substrate system, but both approaches take the substrate effect into the fracture toughness evaluation. The estimated fracture toughness of $\mathrm{WC}-10 \mathrm{Co} 4 \mathrm{Cr}$ coating/1018 low carbon steel substrate system at high temperatures shows that the crystal-structure-based model and microcrack-formation-based models are in good agreement. The fracture toughness of $\mathrm{WC}-10 \mathrm{Co} 4 \mathrm{Cr}$ coating/1018 low carbon steel substrate system remains unchanged until the temperature reaches a critical value, $200 \mathrm{~K}$ for crystal-structure-based model, $150 \mathrm{~K}$ for microcrack-formation-based models. It then increases rapidly in an exponential manner. The room-temperature fracture toughness of the coating/substrate system obtained from all models is about $2.1 \mathrm{MPa} \cdot \mathrm{m}^{1 / 2}$, the fracture toughness at $1000 \mathrm{~K}$ ranges from $8.5669 \mathrm{MPa} \cdot \mathrm{m}^{1 / 2}$ to $11.2399 \mathrm{MPa} \cdot \mathrm{m}^{1 / 2}$ using different models and under different indentation loads. 


\section{Acknowledgements}

I owe my sincere gratitude to my supervisors, Professor Rong Liu, for her constant support and guidance during my research and study at Carleton University. Her expert knowledge and endless patience are invaluable and indispensible for me to finish this thesis.

I would also like to thank my co-supervisor, Dr. Kuiying Chen, at National Research Council Canada. His guidance and knowledge have inspired me to solve the critical problems encountered in the whole research. He was always willing to provide support when I need help.

As well, I want to extend my gratitude to Dr. Xijia Wu at National Research Council Canada, Mathew Yao and Rachael Collier at Kennametal Stellite Inc. in Belleville. I appreciate their assistance in the sample preparation and suggestions for the experiments.

In addition, I would like to thank Xiaozhou Zhang at Carleton University, for his direction and technical experience in the sample preparation and experiments.

Lastly, I would like to thank my parents. They are always there to give me the strongest support. 


\section{Table of Contents}

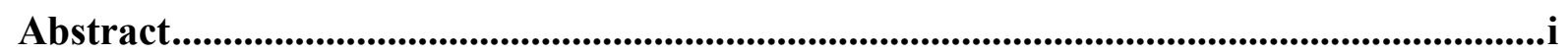

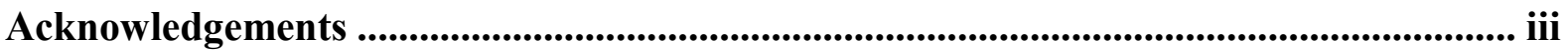

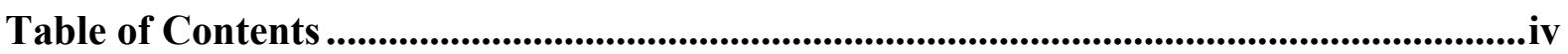

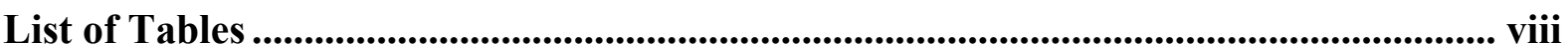

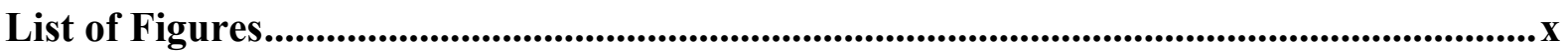

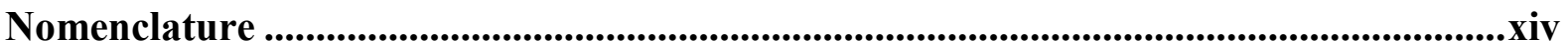

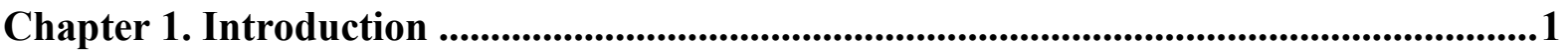

1.1 Research Background and Significance.............................................................. 1

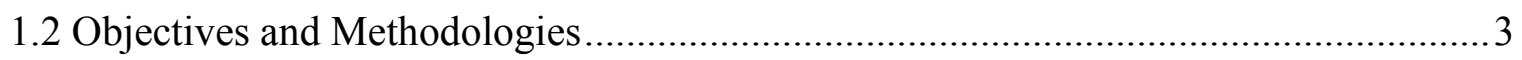

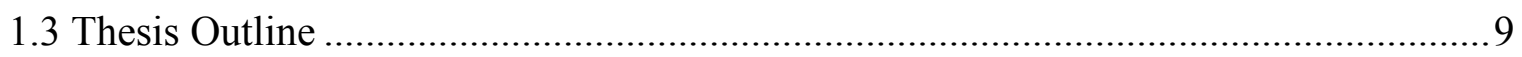

Chapter 2. Literature Review ................................................................................12

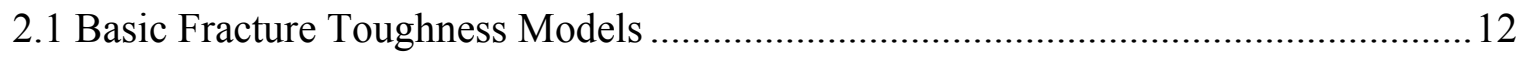

2.2 Fracture Toughness Models at Macroscopic Level ................................................ 17

2.2.1 Fracture toughness models based on indentation test................................... 17

2.2.1.1 Crack types in solids under indentation .............................................. 18

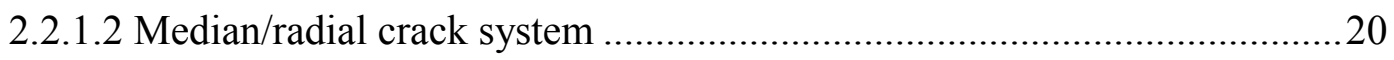

2.2.2 Fracture toughness models based on energy change .....................................24

2.2.3 Fracture toughness models based on stress analysis .....................................2

2.2.4 Temperature-dependent fracture toughness models at macroscopic level .........33 
2.3 Fracture Toughness Models at Microscopic Level .34

2.3.1 The Arrhenius-type equation and rate controlling theory..... .36

2.3.2 Temperature-dependent fracture toughness models at microscopic level

2.4 Closing Remarks .52

Chapter 3. Temperature-dependent Fracture Toughness Models Based on Crystal

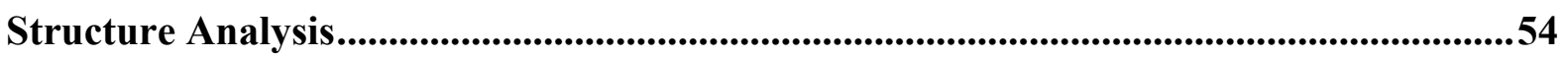

3.1 WC-10Co4Cr Coating/1018 Low Carbon Steel Substrate System...............................54

3.2 Microstructure and Slip System of WC-10Co4Cr Composite...................................... 54

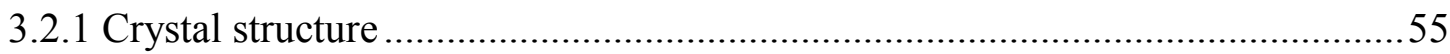

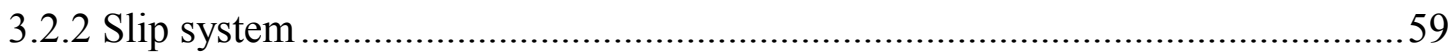

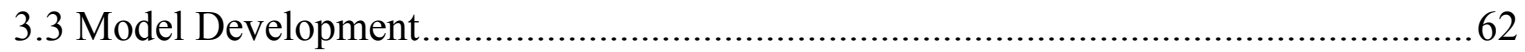

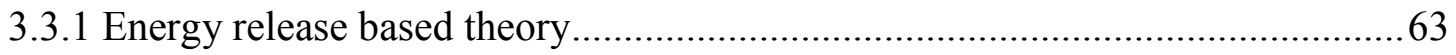

3.3.2 Crack profiles due to Vickers indentation .......................................................69

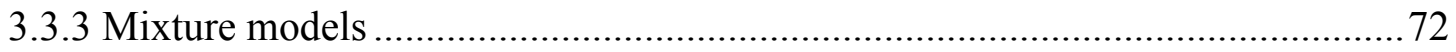

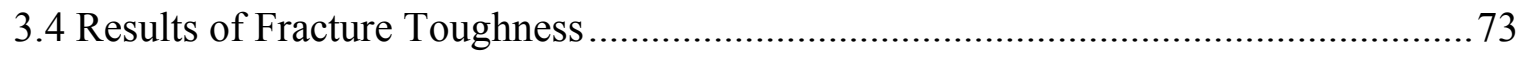

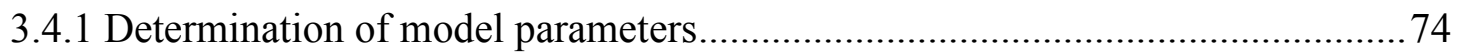

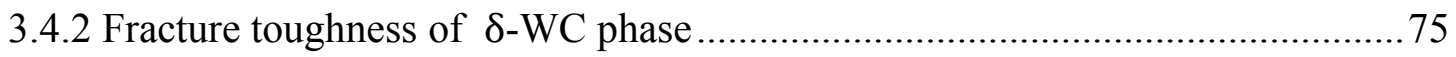

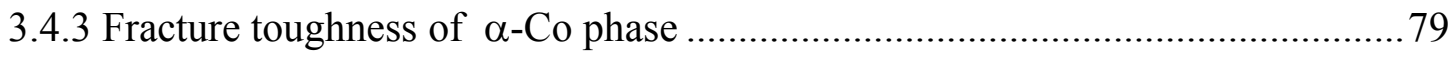

3.4.4 Fracture toughness of WC-10 Co4Cr composite …........................................ 83

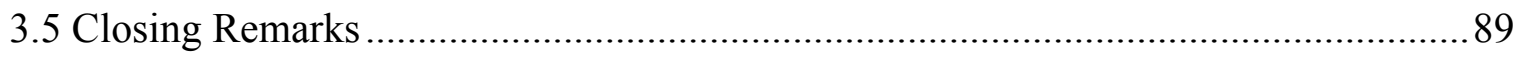


Chapter 4. Temperature-dependent Fracture Toughness Models Based on Microcrack

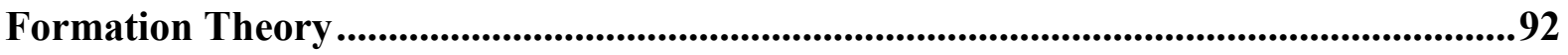

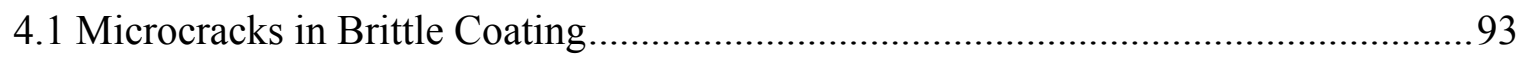

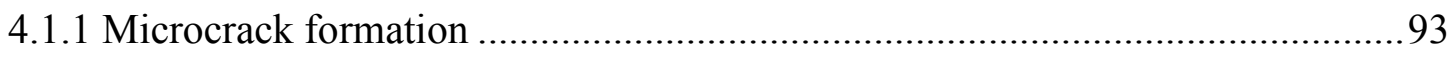

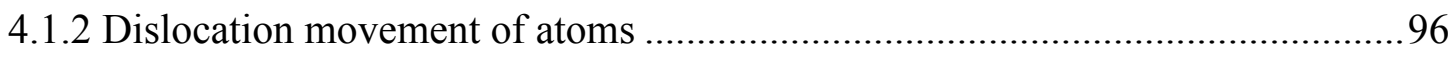

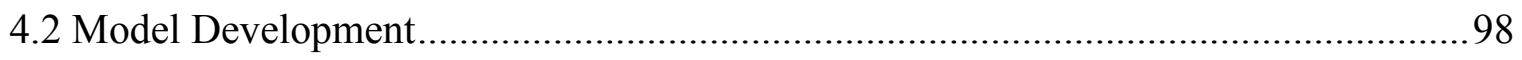

4.2.1 Energy release for microcrack propagation ................................................. 98

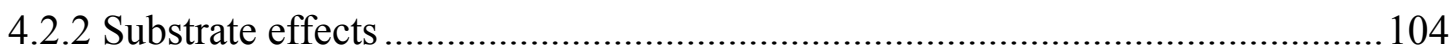

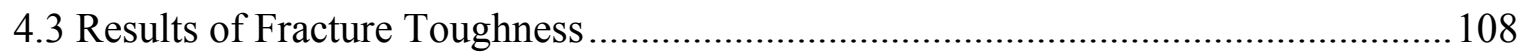

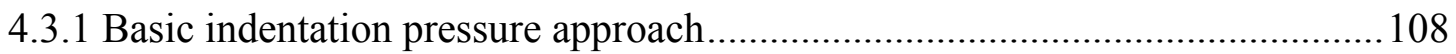

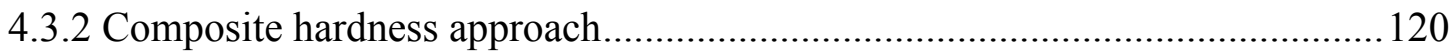

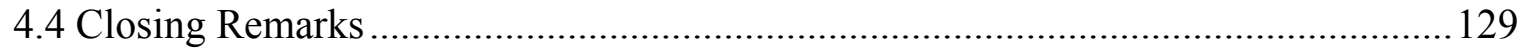

Chapter 5. Discussion of Results.......................................................................................................131

5.1 Comparison of Results between Two Approaches ................................................ 131

5.2 Comparison of Results from Different Models..................................................... 136

Chapter 6. Conclusions and Future Work ....................................................................................140

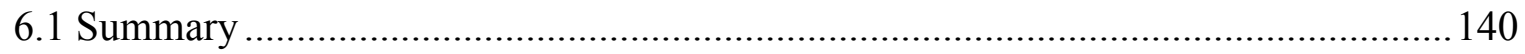

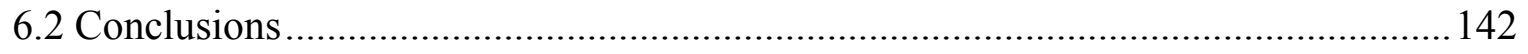

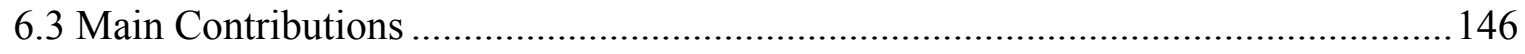

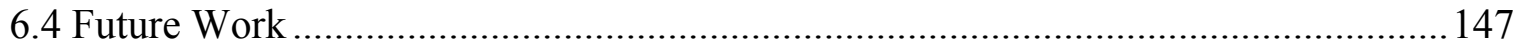

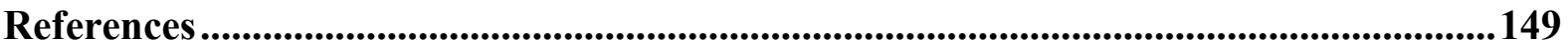


Appendix. 


\section{List of Tables}

Table 3-1. The lattice constant $a$ of $\alpha$-Co phase at different $\mathrm{Cr}$ contents (wt\%) [87] .58

Table 3-2. Lattice parameters of $\delta$-WC and $\alpha$-Co phases 62

Table 3-3. Mechanical properties of WC-10Co4Cr coating and 1018 low carbon steel substrate materials [17] 74

Table 3-4. Required parameters for the temperature-dependent fracture toughness calculation .74

Table 3-5. Calculation results of crystal structure parameters for $\delta$-WC phase .75

Table 3-6. Calculation results of crystal structure parameters for $\alpha$-Co phase .81

Table 4-1. Values of function $F(\Sigma)$ corresponding to different elastic modulus ratios [9].....99

Table 4-2. Average radial crack length $c$ under each indentation load [17]......... 111

Table 4-3. Average indentation diagonal $2 l$ and indentation pressure $p$ under each applied load for the basic indentation pressure approach 112

Table 4-4. Calculated various parameters and fracture toughness for the basic indentation pressure approach

Table 4-5. Calculated parameters based on mean and maximum indentation diagonal lengths for the basic indentation pressure approach 115

Table 4-6. Calculated fracture toughness, $K_{S S}\left(\mathrm{MPa} \cdot \mathrm{m}^{1 / 2}\right)$, based on the basic indentation pressure approach 118

Table 4-7. Average indentation diagonal $2 l$ and indentation pressure $p$ under each 
indentation load for the composite hardness approach

Table 4-8. Calculated various parameters and fracture toughness for the composite hardness approach

Table 4-9. Calculated parameters based on mean and maximum indentation diagonal lengths for the composite hardness approach 124

Table 4-10. Room-temperature fracture toughness based on the same parameter $n$ 126

Table 4-11. Calculated fracture toughness, $K_{S S}\left(\mathrm{MPa} \cdot \mathrm{m}^{1 / 2}\right)$, using the composite hardness approach 127 


\section{List of Figures}

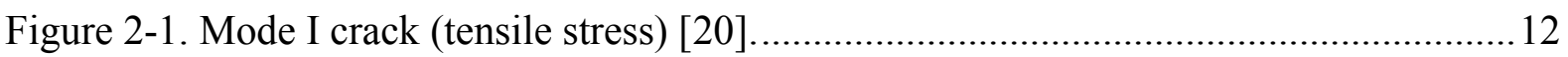

Figure 2-2. A through-thickness crack with length $2 a$ in an infinite plate under tension........ 14

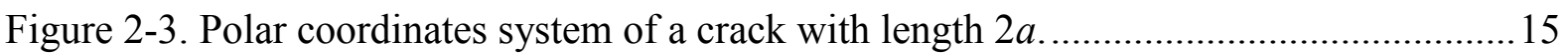

Figure 2-4. Five crack types introduced in a solid body under indentation [16] ................... 18

Figure 2-5. Radial crack geometry due to Vickers indentation: (a) dimension definition, (b) FIB image of indentation surface [13]. 19

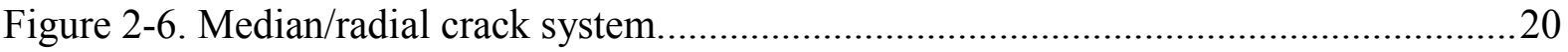

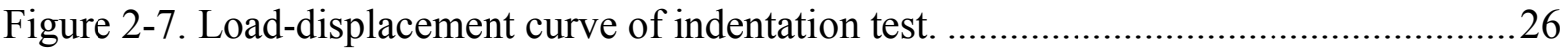

Figure 2-8. Mode I crack in a coating/substrate system: (a) crack configuration, (b) normal and shear stresses.

Figure 2-9. Microcrack formation and the movement of atoms. .36

Figure 3-1. Phase diagram of tungsten carbide WC [81]. .55

Figure 3-2. Hexagonal crystal structure of $\delta$-WC phase. .55

Figure 3-4. Cubic crystal structure of FCC $\alpha$-Co phase. .57

Figure 3-5. Lattice constant $a$ of FCC $\alpha$-Co phase: (a) variation with $\mathrm{Cr}$ content, (b) data from fitting curve. .58

Figure 3-6. Combinations of partial Burgers vectors .59

Figure 3-7. Slip system with Burgers vector $1 / 3[1 \overline{2} 10]$ in $\delta$-WC phase. 61

Figure 3-8. Plastic zone in a coating/substrate system under indentation loading. .66 
Figure 3-9. FIB images of crack profiles in WC-10Co4Cr coating/1018 steel substrate system under Vickers indentation loading: (a) top view, and (b) cross-sectional view [102]. 68 Figure 3-10. Schematic crack profiles in a solid body under Vickers indentation loading: (a) median/radial crack system, (b) indentation and plastic zone geometries. .70

Figure 3-11. The calculated temperature-dependent fracture toughness versus temperature for single-phase $\delta$-WC coating and a measured room-temperature fracture toughness value of bulk WC [117]. .76

Figure 3-12. Comparison between elastic work $J_{s}$ and plastic dissipation energy $J_{d}$ for single-phase $\delta$-WC coating over a temperature range from $1 \mathrm{~K}$ to $1000 \mathrm{~K}$. .78

Figure 3-13. Fracture toughness of WC-Co based alloys: A for $86 \mathrm{WC} 2 \mathrm{TaC} 12 \mathrm{Co}$; B for 85WC9(Ti, Ta,Nb)C6Co; C for 80WC12(Ti,Ta,Nb)C8Co; D for 96WC-4Co [121]. .79

Figure 3-14. Fracture toughness variation of single-phase $\alpha$-Co coating with the ratio of $\kappa / c$. 80

Figure 3-15. Temperature-dependent fracture toughness of single-phase $\alpha$-Co coating. 82

Figure 3-16. Comparison between elastic work $J_{s}$ and plastic dissipation energy $J_{d}$ for single-phase $\alpha$-Co coating over a temperature range from $1 \mathrm{~K}$ to $1000 \mathrm{~K}$ 83

Figure 3-17. Temperature-dependent fracture toughness of WC-10Co4Cr coating/1018 low carbon steel substrate system over a temperature range from $1 \mathrm{~K}$ to $1000 \mathrm{~K}$ with the experimental data of other similar coatings for comparison $[17,118,119]$. 84

Figure 3-18. Temperature-dependent fracture toughness of $\mathrm{WC}-10 \mathrm{Co} 4 \mathrm{Cr}$ coating/1018 low carbon steel substrate system based on two mixture models. .87 
Figure 3-19. Relationship between the surface energy, $\gamma s$, of $\delta$-WC phase and the fracture toughness of (a) $\delta$-WC coating, (b) WC-10Co4Cr coating .88

Figure 3-20. Relationship between $\gamma_{S} / U_{P-N}$ and the fracture toughness of $\delta$-WC coating.. 89 Figure 4-1. Schematic illustration of radial cracks in a brittle coating under Vickers indentation.

Figure 4-2. Schematic illustration of the crack tip of macrocrack and microcrack formation in brittle materials. .94

Figure 4-3. Schematic illustration of microcrack formation and the movement of atoms. 97

Figure 4-4. $F(\Sigma)$ versus the elastic modulus ratio $\Sigma$ : (a) graph, (b) polynomial fitting data. 100

Figure 4-5. A constant shear stress at the coating/substrate interface. 101

Figure 4-6. Indentation diagonal $2 l$ Parameters versus indentation load $P$ for the basic indentation pressure approach: (a) indentation diagonal $2 l$, (b) parameter $n$, (c) indentation pressure $p,(\mathrm{~d})$ microcrack density $\rho_{c}$ 116 Figure 4-7. Temperature-dependent fracture toughness of the composite coating/substrate system under different indentation loads using the basic indentation pressure approach: (a) $196 \mathrm{~N}$, (b) $294 \mathrm{~N}$, (c) $392 \mathrm{~N}$, (d) $490 \mathrm{~N}$, (e) $720 \mathrm{~N}$. 119

Figure 4-9. Temperature-dependent fracture toughness under different indentation loads using the composite hardness approach: (a) $196 \mathrm{~N}$, (b) $294 \mathrm{~N}$, (c) $392 \mathrm{~N}$, (d) $490 \mathrm{~N}$, (e) $720 \mathrm{~N} \ldots . . .128$ Figure 5-1. Indentation pressure $p$ versus applied load $P$. 132

Figure 5-2. Parameters versus indentation load $P$ : (a) microcrack density $\rho_{c}$, (b) parameter $n$. 
Figure 5-3. Comparison of calculated fracture toughness for the composite coating/substrate system between two approaches: (a) $298 \mathrm{~K}$, (b) $400 \mathrm{~K}$, (c) $600 \mathrm{~K}$, (d) $800 \mathrm{~K}$, (e) $1000 \mathrm{~K} . \ldots .134$ Figure 5-5. Maximum and minimum temperature-dependent fracture toughness obtained from three models. 138 


\section{Nomenclature}

$a$

$a_{c} \quad$ half diagonal of indentation impression

$a_{0} \quad$ lattice constant

A

$A^{*}$

$b$

$c$

C

$C_{d}$

$d$

$D$

E

$E^{c}$

$E^{S}$

$\Delta E$

$f$

$F(\Sigma) \quad$ elastic modulus ratio function

$\Delta F$

G crack dimension in an infinite plate

radial crack area

dislocation area

Burgers vector

radial crack length

crack width

diameter of the crack

indentation depth

crack spacing

Young's modulus

Young's modulus of the coating

Young's modulus of the substrate

general energy change

geometry related factor for $a_{c}$ and $h$

intrinsic barrier energy

strain energy release rate 


\begin{tabular}{|c|c|}
\hline$G_{a}$ & crack arrest energy \\
\hline$G_{C}$ & critical strain energy release rate \\
\hline$G_{e}$ & strain energy release rate in Hu's model \\
\hline$G_{i}$ & crack initiation energy \\
\hline$G_{S S}$ & strain energy release rate for mode I crack \\
\hline$\Delta G$ & Gibbs free activation energy \\
\hline$\Delta G(W)$ & thermal energy change \\
\hline$h$ & radius of plastic zone \\
\hline$H$ & hardness \\
\hline$\Delta H^{*}$ & activation enthalpy \\
\hline$J_{d}$ & plastic component $J$ integral \\
\hline$J_{s}$ & elastic component $J$ integral \\
\hline$k$ & Boltzmann constant \\
\hline$K_{b}$ & fracture toughness of brittle phase \\
\hline$K_{c}$ & composite fracture toughness \\
\hline$K_{d}$ & fracture toughness of ductile phase \\
\hline$K_{I}$ & stress intensity factor \\
\hline$K_{I c}$ & fracture toughness \\
\hline$K_{S S}$ & fracture toughness for through thickness cra \\
\hline$l$ & total length of the dislocation line \\
\hline$l_{d}$ & distance between dislocation \\
\hline
\end{tabular}


length of dislocation line,

$L$

characteristic length of microcrack

m

geometry related factor for $b$ and $\delta$

M

Taylor orientation factor

$n$

scale-linking parameter

$N$

total amount of activation points

$p$

Indentation pressure

$P$

indentation load

$q$

slip plane spacing

$Q_{c}$

activation energy

$r$

distance from crack tip

$R$

gas constant

$S$

temperature-dependent rate constant

$\dot{S}$

strain rate

$t$

coating thickness

$T$

absolute temperature

$U$

strain energy change during crack propagation

$U_{P-N}$

Peierls-Nabarro (P-N) barrier energy

$U_{f r}^{c}$

energy dissipation of chipping behavior

$\Delta U$

total energy change during crack propagation

$\Delta U_{e}$

strain energy change 
$\Delta U_{s}$

$v$

$v_{c}$

$v_{d}$

$v^{a}$

$v^{*}$

V

$V_{f}$

$V_{p}$

$V^{*}$

W

$W_{S}$

$W(\sigma)$

$\Delta W$

$\Delta W_{d}$

$x^{*}$

$X$

Y

Z

$\alpha$

$\beta$ strain energy variation for interface sliding

crack velocity

crack growth rate

dislocation velocity

apparent activation volume

activation volume

indenter contact volume

volume fraction of the brittle phase

volume of plastic zone

critical volume

energy release during crack propagation

surface energy change during crack propagation

work done by applied stress

total work done by the applied load

energy dissipation caused by interface sliding

thermal activation distance

parameter decided by of crack profiles and materials' properties

yield strength

shape factor for radial cracks

ratio of crack depth and coating thickness

angle in polar coordinates system 


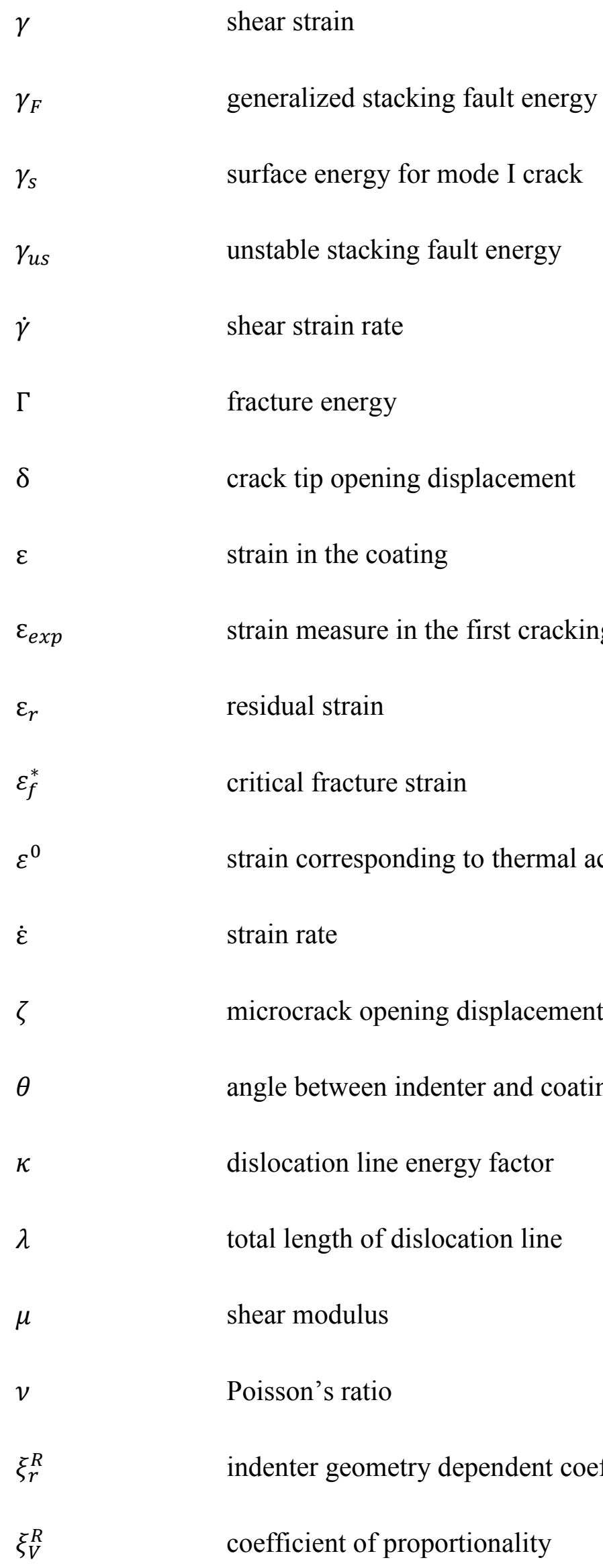




\begin{tabular}{|c|c|}
\hline$\rho$ & dislocation density \\
\hline$\rho_{c}$ & microcrack areal density \\
\hline$\rho_{m}$ & mobile dislocation density \\
\hline$\sigma$ & total stress \\
\hline$\sigma_{a p p}$ & applied stress \\
\hline$\sigma_{c}$ & critical stress for crack propagation \\
\hline$\sigma_{c S}$ & critical stress \\
\hline$\sigma_{c s f}$ & critical fracture stress \\
\hline$\sigma_{\text {int }}$ & internal stress \\
\hline$\sigma_{r}$ & residual compressive stress \\
\hline$\sigma_{t c s}$ & theoretical cohesive strength \\
\hline$\left(\sigma_{x x}, \sigma_{y y}, \sigma_{x y}\right)$ & stress component at crack tip \\
\hline$\sigma_{Y}$ & yield stress \\
\hline$\sigma_{0}$ & residual stress \\
\hline$\sigma_{\infty}$ & applied tension \\
\hline$\sigma_{y y}^{\infty}$ & remote tension \\
\hline$\Sigma$ & elastic modulus ratio \\
\hline$\tau$ & applied shear stress \\
\hline$\tau_{y}$ & shear yield stress \\
\hline$\tau_{\mu}$ & intrinsic barrier shear stress \\
\hline$\tau_{e}^{*}$ & effective shear stress \\
\hline
\end{tabular}


$v$

$v_{D}$

$v_{0}$

$v^{\prime}$

$\Phi$

$\chi_{r}$

$\psi$

$\Psi$

$\Omega$

$\Omega_{c}$ frequency factor

Debye frequency

frequency related factor

activation frequency in the dislocation area

constraint factor

residual component

lattice phase angle

half-angle of indenter

atomic volume

critical cracking number 


\section{Chapter 1. Introduction}

\subsection{Research Background and Significance}

Coating technology is undergoing a rapid development, since materials are required to exhibit better mechanical properties and corrosion resistance when they are applied in tough environments [1]. High velocity oxy fuel (HVOF) is one of the most commonly used coating processing methods and has many of benefits because of the high velocity of powders and the low temperature during the deposit process. This processing method can offer better adhesion and higher hardness to coating, increase the possibility to avoid brittle phases, and the wear resistance can be enhanced as well [2].

The tungsten carbide, WC-based cermet coatings have been well developed and extensively applied to the surfaces of components serving in aviation, aerospace, automobile and other advanced industries in order to improve the components' resistance against abrasion, fretting, erosion and other problems encountered during applications [3-6]. The WC-Co thermal sprayed coatings are known for their extraordinary integration of fracture toughness and hardness [7]. Corrosion resistance, another critical criterion for coating selection and application, could be improved by introducing chromium to WC-Co coatings [8]. These brittle films attached to substrates can provide superior protection and prolong the service life of the components employed in various industrial applications $[9,10]$. 
Materials are frequently required to work under high tensile stress conditions, and the fracture toughness, $K_{I C}$, of the coating/substrate system is a critical parameter for material selection and assessment. In such situations, cracks propagate and cause fracture or fatigue of components. Fracture toughness of brittle coating/substrate systems describes the performance of the system in resisting the crack propagation under different forms of impact and loading cycles $[11,12]$. Among the test methods currently used to measure the fracture toughness of coating/substrate systems, the indentation test is the most widely applied technique owing to its simplicity, affordability and reliability $[12,13]$.

Apparatuses are required to operate at high temperatures in many industries, especially in energy, aerospace, automotive, chemical and manufacturing industries. Meanwhile, the mechanical properties, including fracture toughness, of bulk materials and coating/substrate systems could be significantly influenced by temperature variation. In recent years, thermodynamics and micromechanics have been applied to taking the effect of temperature into the study of materials' mechanical properties, and several temperature-dependent fracture toughness models have been developed for bulk materials, but some critical parameters in these models are obtained with large approximations and do not have universality. On the other hand, the study of temperature-dependent fracture toughness of coating/substrate systems has rarely been published, since the experimental data at high temperatures are difficult to obtain due to the limitations of the existing testing facilities and methodologies [14]. For coating applications at high temperatures, therefore, an effective 
means is necessary to assess the strength and fracture toughness of coating/substrate systems in such environments.

\subsection{Objectives and Methodologies}

This research is aimed to study the fracture behavior of a brittle coating/ductile substrate system (The WC-10Co4Cr coating/1018 low carbon steel substrate) from room temperature to $1000 \mathrm{~K}$. This coating/substrate system is selected for four reasons. First, it is a typical brittle coating/ductile substrate system. Second, this coating is used for resisting severe wear in various fields owing to the high hardness ( $\sim \mathrm{HV} 1300)$ and excellent wear resistance of WC-10Co4Cr. Third, this coating/substrate system has been extensively studied at room-temperature conditions so that the required properties of the coating and substrate materials and the experimental/simulations results of this system are well known. Finally, fourth, although the temperature-dependent fracture toughness models are developed for WC-10Co4Cr coating/1018 low carbon steel substrate system, they can also be applied to other brittle coating/ductile substrate systems as long as the required material properties and experimental data are obtainable.

First, based on the Arrhenius-type equation, the temperature-dependent fracture toughness model developed at microscopic level [14] and the median/radial crack propagation models proposed by Lawn et al. [15] and Cook et al. [16], an analytical model is obtained for the temperature-dependent fracture toughness estimation of $\mathrm{WC}-10 \mathrm{Co} 4 \mathrm{Cr}$ coating/1018 low 
carbon steel system by substitutes the area of plastic zone in the composite coating for that in bulk materials to fit the type and geometry of the cracks generated under indentation loading. The dislocation density variation with the indentation load is integrated into the fracture toughness calculation using the model developed in Durst's work [17]. The phase diagrams and microstructures of the two phases in the composite coating are analyzed and discussed. The estimated room-temperature fracture toughness is compared with the reported results in literature to verify the assumptions and parameters in the formulations of the model development.

Second, based on the microcrack formation theory, Arrhenius-type equation and the fracture toughness model developed in Hu's work [9], the temperature-dependent fracture toughness models for WC-10Co4Cr coating/1018 low carbon steel system are proposed using the basic indentation pressure approach and the composite hardness approach, respectively. Two parameters, $m$ and $n$, are introduced in these models. The parameter $m$ relates the total growth of microcrack in terms of crack tip opening displacement to the magnitude of dislocation movement of atoms in terms of Burgers vector. The scale-linking parameter $n$ relates the micro-level energy barrier to the strain energy release rate, which is to minimize the load effect at room temperature, similar to the function of crack shape influence factor. The data of the radial crack length and the indentation impression diagonal are required for these models, which were obtained by Demidova [18]. 
The WC-10Co4Cr coating under study, is a composite material consisting of WC powder (86 wt $\%$ or 76.42 vol $\%$ ) and CoCr powder (10 wt $\%$ or 15.77 vol $\%$ Co and $4 \mathrm{wt} \% \mathrm{Cr}$ or 7.81 vol\%). The mixed powder is sprayed to the surface of AISI 1018 low carbon steel substrate via high velocity oxygen fuel (HVOF) method. The stoichiometric formula of WC powder is $6.13 \mathrm{wt} \% \mathrm{C}$ and $93.87 \mathrm{wt} \% \mathrm{~W}$. According to the composition and phase diagrams, this thermal sprayed coating can be treated as a two-phase composite coating which consists of hexagonal $\delta$-WC and FCC $\alpha$-Co phases.

In the indentation test-based fracture toughness model developed by Lawn et al. [15], the radial cracks initiated in the loading process would propagate on the coating surface and downward to the coating/substrate interface, and be fully developed during the unloading period. The major driving force for the radial crack extension is the residual stresses stored in the plastic zone during the loading period, while the elastic driving force is totally subordinated to the plastic driving force in this process. Other types of cracks observed in the WC-10Co4Cr coating/1018 low carbon substrate system under indentation loading, such as median crack and edge crack, are formed and reach their full lengths during the loading process. Based on this crack initiation and propagation analyses, the temperature-dependent fracture toughness can be evaluated through computing the total dislocation energy stored in the plastic zone which is responsible for radial crack development during the unloading process. Therefore, the profiles of radial cracks developed during the unloading period are used to investigate the fracture toughness of the composite coating/substrate system. A series 
of indentation tests with different applied load was performed on WC-10Co4Cr coating/1018 low carbon substrate at room temperature in Demidova's work [18], and all the crack profiles were obtained using an optical microscope Olympus PMG-3 and a Micrion 2500 Focused Ion Beam (FIB) microscope system. These experimental data are applied in this research to establish the relationship between indentation load and the fracture toughness of the composite coating/substrate system.

The strain energy release rate used to measure the energy dissipation per unit area of instantly formed crack can be divided into two parts, the work done by elastic deformation, $J_{s}$, and done by plastic deformation, $J_{d}$. The $J_{d}$ expressed using the Arrhenius-type equation and rate controlling theory is responsible for taking the temperature effect into the fracture toughness evaluation. This formulation is utilized to study the temperature-dependent fracture behavior of the composite coating/substrate system in this research.

In the unloading process, numerous microcracks are generated from each corner of indentation impression and merge together to form radial cracks along the indentation diagonals under the tension of residual stresses. According to Ortiz's work [19], in brittle materials, the microcracks could be assumed to be evenly spaced and have the same length during the crack extension. Meanwhile, the microcrack opening displacement increases gradually during the crack propagation process, since the microcracks in the front of the macrocrack tip become the new crack tip when they are merged into the macrocrack. As a 
result, the crack tip opening displacement (CTOD), $\delta$, could be used to measure the total growth of a microcrack in tensile direction, and the dislocation movement of atoms could be used to describe the microcrack formation and propagation. Based on this concept, microcrack-formation-based fracture toughness models are developed in this research to investigate the temperature-dependent fracture toughness of $\mathrm{WC}-10 \mathrm{Co} 4 \mathrm{Cr}$ coating/1018 low carbon steel substrate system.

The main tasks for the fulfillment of the objectives are summarized as follows:

1. Investigate the microstructure and lattice parameters of each phase in the composite coating based on their chemical compositions and phase diagrams, and determine their Burgers vectors and interplanar spacing for fracture toughness formulations.

2. Examine the radial crack propagation process based on Lawn's crack model [15] and Cook's work [16], analyze the plastic zone and determine the dislocation density of the composite coating/substrate system.

3. Modify a temperature-dependent fracture toughness model where the model parameters are fitted to the crack type and geometries in the composite coating/substrate system under indentation loading. Meanwhile, some critical parameters obtained in the original model are examined and re-calculated for the specific coating/substrate system. 
4. Compare the estimated fracture toughness with the existing results to examine the assumptions used in the model formulation. In order to study the temperature-dependent fracture behavior of $\mathrm{WC}-10 \mathrm{Co} 4 \mathrm{Cr}$ coating, the strain energy release rates of elastic and plastic deformations obtained from the modified model are plotted against temperature.

5. Develop new temperature-dependent fracture toughness models based on microcrack formation and propagation during indentation. Calculate the microcrack density using the crack spacing and residual stress equations. Investigate the substrate effect on the system through indentation pressure calculation, and analyze the difference between two indentation pressure calculation approaches.

6. Compare the critical parameters and fracture toughness obtained from these two approaches. Examine the experimental data obtained from the indentation tests in Demidova's work [18], analyze the estimated fracture toughness as a function of applied indentation load.

7. Investigate the fracture toughness variation versus temperature, compare the temperature-dependent fracture toughness derived from different models developed in this research, and discuss the consistency and deviation. 


\subsection{Thesis Outline}

Chapter 1 introduces the background, significance and the objectives of this research. The main tasks involved and the methodologies utilized to fulfill the objectives are indicated. The organization of this thesis is outlined.

Chapter 2 reviews the existing fracture toughness models and their developments. The indentation technique, crack types and the fracture toughness models based on indentation tests are reviewed. The energy-based and stress-based fracture toughness models for brittle coating/ductile substrate systems are discussed. The development and application of strain and crack growth rate models based on the Arrhenius-type equation and rate controlling theory are of particular concerned, with a focus on the temperature-dependent fracture toughness models. The shortcomings and limitations of the existing fracture toughness models are discussed.

Chapter 3 presents the investigations into the microstructures and lattice parameters of each phase in the composite coating based on their compositions and phase diagrams. The radial crack propagation process, the plastic zone dimensions and the dislocation density of the composite coating/substrate system are analyzed. An existing temperature-dependent fracture toughness model is modified to fit the crack type and geometries generated in the coating/substrate system under indentation, and some critical parameters obtained from initial approximation in the original model are re-calculated for this specific coating/substrate 
system. The calculated fracture toughness is compared with the reported results in literature to examine the assumptions made in this simulation and the calculated parameters from the modified models. Two mixture methods for integrating the fracture toughness of the individual phases in the $\mathrm{WC}-10 \mathrm{Co} 4 \mathrm{Cr}$ composite coating are compared. In order to study the fracture behavior of $\mathrm{WC}-10 \mathrm{Co} 4 \mathrm{Cr}$ coating at high temperatures, the strain energy release rate of elastic and plastic deformations obtained from the modified models are plotted against temperature.

Chapter 4 describes the analyses of the microcrack formation and extension process in WC-10Co4Cr coating/1018 low carbon steel substrate system under indentation loading at high temperatures. These include investigating two indentation pressure calculation approaches, and developing new temperature-dependent fracture toughness models. The experimental data obtained from indentation tests at room temperature are analyzed, the critical parameters and the fracture toughness of the composite coating/substrate system are calculated using the two approaches.

Chapter 5 presents the comparison of the fracture toughness estimated for $\mathrm{WC}-10 \mathrm{Co} 4 \mathrm{Cr}$ coating/1018 low carbon steel substrate system using the microcrack-formation-based models from the two indentation pressure calculation approaches, and also presents the comparison of the fracture toughness results from different models developed in this research. The graphs of the fracture toughness versus temperature are discussed. 
Chapter 6 summarizes the main activities and outcomes of this research. The conclusions are presented. The main contributions are highlighted. The future work is proposed. 


\section{Chapter 2. Literature Review}

\subsection{Basic Fracture Toughness Models}

Fracture toughness, $K_{I c}$, is the ability of materials to resist the crack propagation under cyclic load conditions. For indentation tests, $K_{I c}$ is the ability of materials to resist the crack propagation under single indentation load. The concept of energy-based fracture mechanics was first proposed by Griffith [20]. The theoretical cohesive strength, $\sigma_{t c s}$, of brittle glass was related to material's surface energy, $\gamma_{s}$, and lattice constant, $a_{0}$ :

$$
\sigma_{t c s}=\sqrt{\frac{2 E \gamma_{s}}{a_{0}}}
$$

where $E$ is Young's modulus. However, Eq. (2-1) was developed for the non-crack condition, which cannot be used to predict the crack propagation behavior of pre-existing cracks.

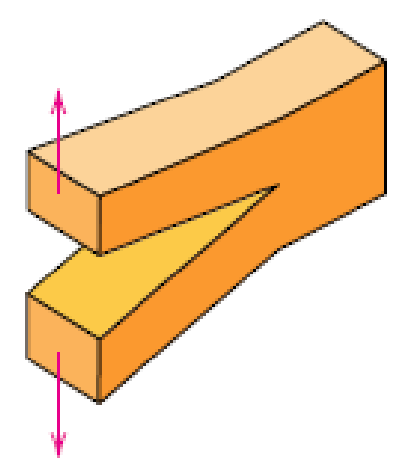

Figure 2-1. Mode I crack (tensile stress) [21].

For mode I crack, which is under tensile stress, as shown in Figure 2-1, Griffith stated that the actual critical fracture stress might be significantly influenced by pre-existing cracks, and the crack propagation occurred when the total amount of reduced strain energy in the system 
exceeded the increased surface energy. By considering a straight through-thickness crack with length $2 a$ in an infinite plate, which is subject to a remote tension, $\sigma_{y y}^{\infty}$, as shown in Figure 2-2, the total amount of released energy, $W$, during the crack propagation is given by:

$$
W=\frac{\pi a^{2} \sigma_{y y}^{\infty}\left(1-v^{2}\right)}{E}
$$

for plane strain, and

$$
W=\frac{\pi a^{2} \sigma_{y y}^{\infty}}{E}
$$

for plane stress, where $v$ is Poisson's ratio, and the total amount of increased surface energy, $W_{s}$, is given by:

$$
W_{S}=4 \gamma a
$$

where $\gamma$ is surface energy. Then, the critical fracture stress, $\sigma_{c s f}$, can be obtained through the equation:

$$
\frac{2 \pi a \sigma_{y y}^{\infty}\left(1-v^{2}\right)}{E} \mathrm{~d} a=4 \gamma \mathrm{d} a
$$

for plane strain, and

$$
\frac{2 \pi a \sigma_{y y}^{\infty}}{E} \mathrm{~d} a=4 \gamma \mathrm{d} a
$$

for plane stress. Therefore, the critical fracture stress, $\sigma_{c s f}$, is:

$$
\sigma_{c s f}=\sqrt{\frac{2 E \gamma}{\pi a\left(1-v^{2}\right)}}
$$

and

$$
\sigma_{c s f}=\sqrt{\frac{2 E \gamma}{\pi a}}
$$


for plane stain and plane stress, respectively. As a result, the difference between the theoretical crack stress and the test result can be explained, and the strain energy release rate, $G$, for a single crack tip under plane strain condition can be defined as

$$
G=\frac{1}{2} \frac{\mathrm{d} W}{\mathrm{~d} a}=\frac{\pi a \sigma_{y y}^{\infty}\left(1-v^{2}\right)}{E}
$$

The energy-based fracture criterion was first defined in Irwin's work [22].

$$
G \geq G_{c}=2 \gamma_{s}
$$

where $G_{c}$ is the minimum value of $G$ for crack extension.

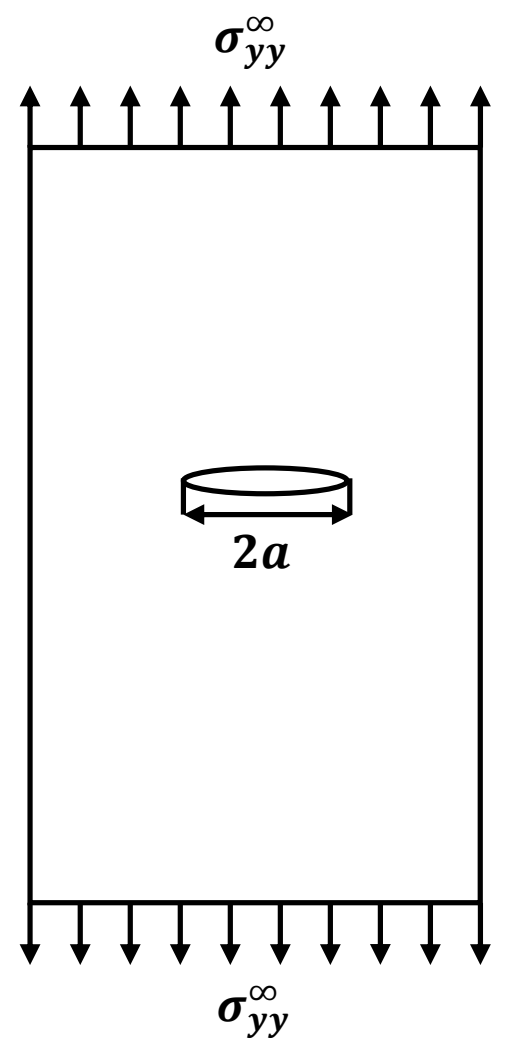

Figure 2-2. A through-thickness crack with length $2 a$ in an infinite plate under tension. 
To obtain an explicit expression of stress intensity factor, a polar coordinates system is built for a crack with length $2 a$, as shown in Figure 2-3:

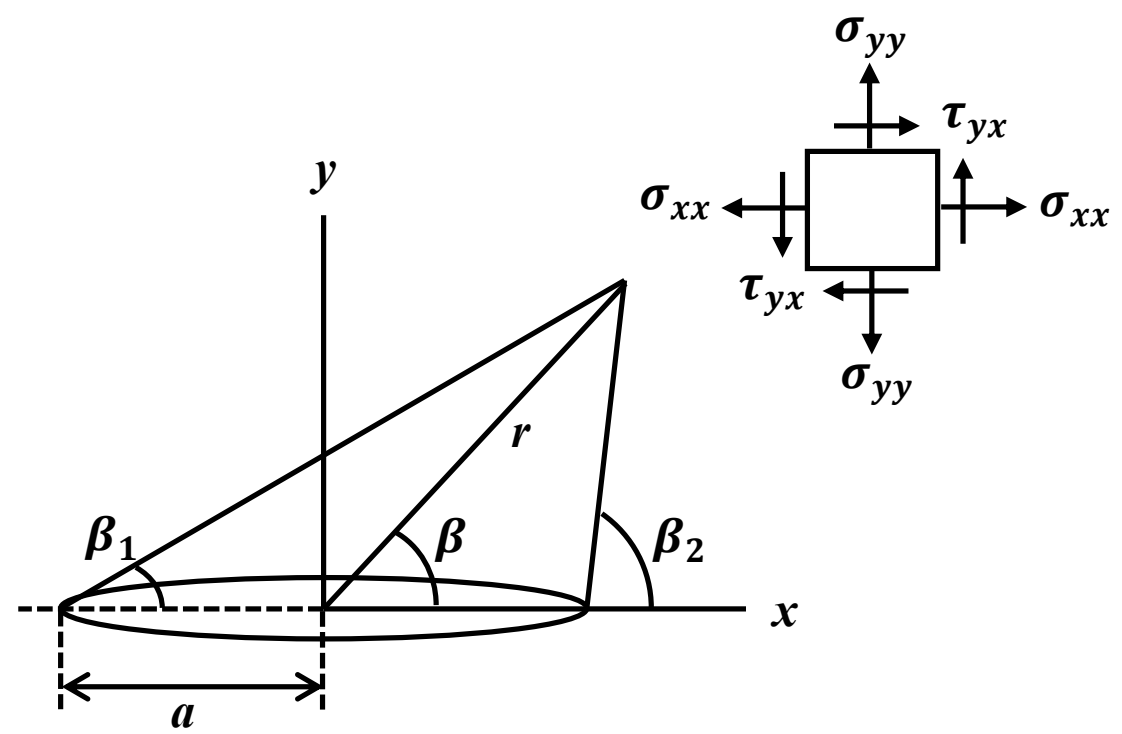

Figure 2-3. Polar coordinates system of a crack with length $2 a$.

The stress intensity factor, $K_{I}$, near the crack tip can be described by using three stress components which are given by [23]:

$$
\begin{aligned}
& \sigma_{x x}=\frac{K_{I}}{\sqrt{2 \pi r}} \cos \frac{1}{2} \beta\left(1-\sin \frac{1}{2} \beta \sin \frac{3}{2} \beta\right) \\
& \sigma_{y y}=\frac{K_{I}}{\sqrt{2 \pi r}} \cos \frac{1}{2} \beta\left(1+\sin \frac{1}{2} \beta \sin \frac{3}{2} \beta\right) \\
& \sigma_{x y}=\frac{K_{I}}{\sqrt{2 \pi r}} \sin \frac{1}{2} \beta \cos \frac{1}{2} \beta \cos \frac{3}{2} \beta
\end{aligned}
$$


To estimate the stress intensity factor, the stress component $\sigma_{y y}$ is applied with the condition of $\beta=0$, then the stress component $\sigma_{y y}$ and the stress intensity factor, $K_{I}$, can be written as:

$$
\begin{gathered}
\sigma_{y y}=\frac{K_{I}}{\sqrt{2 \pi r}} \\
K_{I}=\sigma_{y y} \sqrt{2 \pi r}
\end{gathered}
$$

where $r$ is the distance from a crack tip. Another expression of stress component $\sigma_{y y}$ can be derived via Westergaard function method $[24,25]$ :

$$
\sigma_{y y}=\frac{\sigma_{y y}^{\infty} \sqrt{a}}{\sqrt{2 \pi r}} \cos \frac{1}{2} \beta\left(1+\sin \frac{1}{2} \beta \sin \frac{3}{2} \beta\right)
$$

where $\sigma_{y y}^{\infty}$ is the remote tensile stress, as shown in Figure 2-2. When $\beta=\beta_{1}=\beta_{2}=0$, $\sigma_{y y}$ can be reduced into:

$$
\sigma_{y y}=\frac{\sigma_{y y}^{\infty} \sqrt{a}}{\sqrt{2 \pi r}}
$$

Comparing Eq. (2-9) with Eq. (2-10), the stress intensity factor, $K_{I}$, can be rewritten as:

$$
K_{I}=\sigma_{y y}^{\infty} \sqrt{\pi a}
$$

In this situation, the crack propagates when the stress intensity factor, $K_{I} \geq K_{I c}$. Therefore, the fracture toughness for plane strain condition is expressed as:

$$
\begin{gathered}
K_{I c}=\sigma_{c} \sqrt{\pi a} \\
\sigma_{c}=\sqrt{\frac{2 E \gamma_{s}}{\pi a\left(1-v^{2}\right)}}
\end{gathered}
$$

where $\sigma_{c}$ is the critical stress for crack propagation [25]. 
According to Eq. (2-7) and Eq. (2-12) the fracture toughness, $K_{I C}$, for plane strain condition can be described as:

$$
K_{I c}=\sqrt{\frac{G E}{\left(1-v^{2}\right)}}
$$

In terms of the linear elastic fracture mechanics, the fracture toughness, $K_{I c}$, can also be expressed as:

$$
K_{I c}=X \sigma_{c} \sqrt{\pi a}
$$

where $X$ is a variable parameter determined by crack profiles, materials' dimensions and properties, load types and test methods [21].

\subsection{Fracture Toughness Models at Macroscopic Level}

For fracture toughness evaluation, numerous models have been developed based on experiments and measurements at macroscopic level.

\subsubsection{Fracture toughness models based on indentation test}

Several techniques are currently applied to the fracture toughness estimation of coating/substrate systems, including bending, buckling, scratching and indentation techniques. Among these test methods, indentation technique is the most widely used method for such an application. It is also quite often applied to hardness evaluation of both bulk materials and coating/substrate systems owing to its simplicity, affordability and reliability. 


\subsubsection{Crack types in solids under indentation}

For different indenter geometries and material properties, five crack types were observed in indentation tests and were summarized by Cook and Pharr [16], as shown in Figure 2-4.

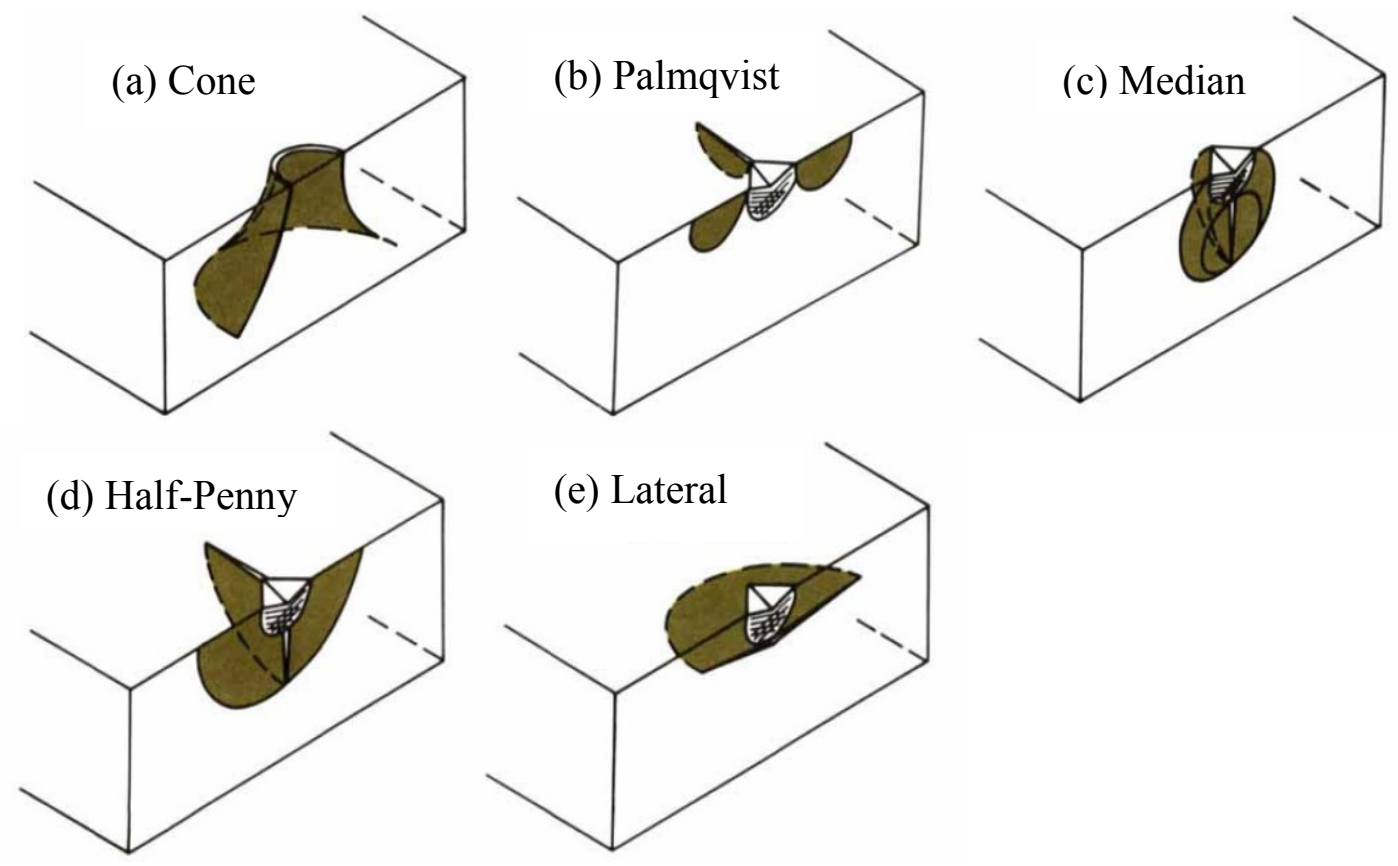

Figure 2-4. Five crack types introduced in a solid body under indentation [16].

As shown in Figure 2-4(b), Palmqvist crack is also recognized as radial crack, which is one of the most common crack types generated in WC-based coatings under indentation loading. In addition to radial crack, median crack is another crack type that is generally observed in this type of coating. Half-penny cracks can be formed when both median cracks and radial cracks appear in coating/substrate systems. Lateral cracks could also be observed in WC-based coatings as well. Different from median cracks which are generated and fully developed during the loading process, radial cracks are initiated in the loading period, but their growth is restrained by the elastic component formed outside the deformation zone in this period. 
Therefore, radial cracks propagate and reach their full length during the unloading process [15].

Among various indenter geometries, the sharp edge Vickers indenter and Berkovich indenter are commonly employed in hardness and fracture toughness determinations of brittle coatings [27]. The crack profiles for fracture toughness estimation of WC-10Co4Cr coating/1018 low carbon steel substrate system in this research are obtained from Vickers indentation tests, as shown in Figure 2-5.

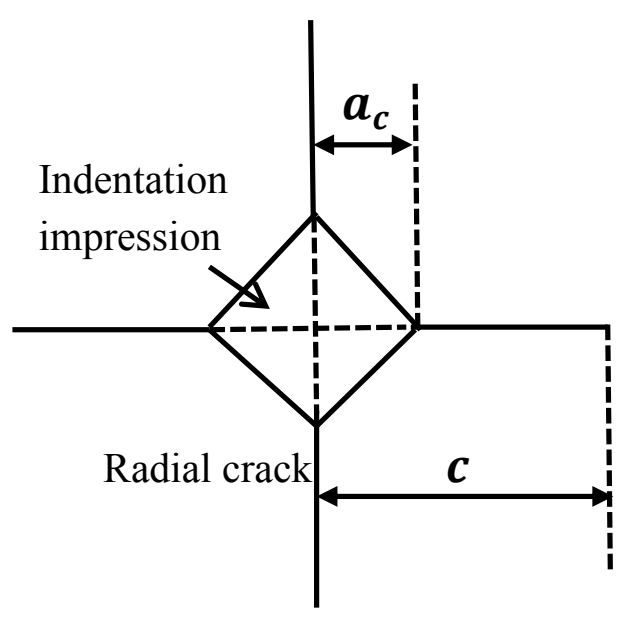

(a)

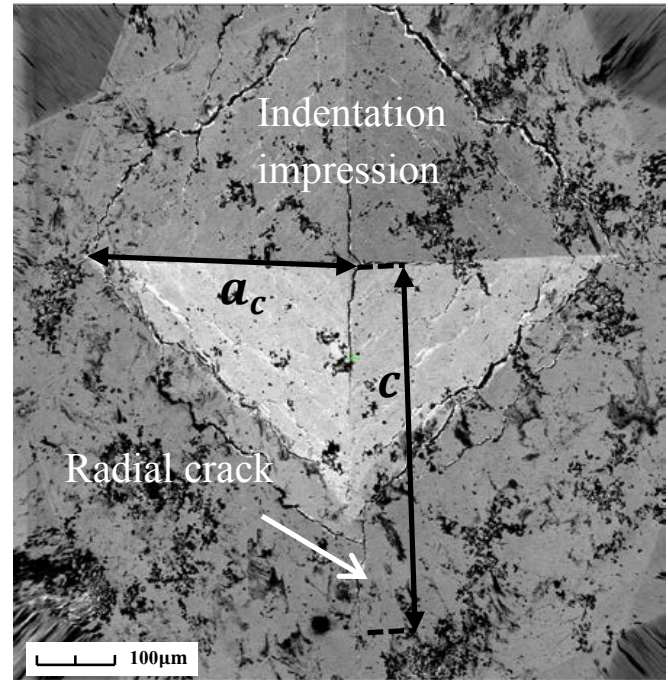

(b)

Figure 2-5. Radial crack geometry due to Vickers indentation: (a) dimension definition, (b) FIB image of indentation surface [13]. 


\subsubsection{Median/radial crack system}

Median/radial crack system is also recognized as half-penny crack, which is formed from downward extension of radial crack or upward propagation of median crack or the combination of two crack types, as shown in Figure 2-6.

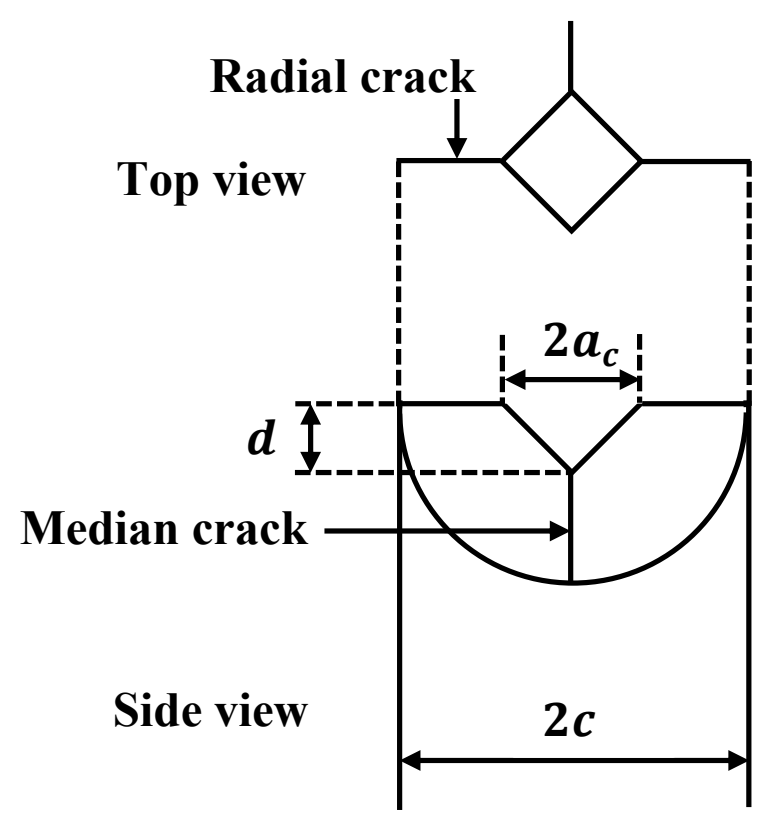

Figure 2-6. Median/radial crack system.

Using Vickers indentation technique, Evans and Charles [28] conducted a series of tests on different materials, including WC-12Co. The calculated fracture toughness of WC-12Co composite was $16 \mathrm{MPa} \cdot \mathrm{m}^{1 / 2}$, and a general expression for estimating the fracture toughness of median/radial crack system in ceramic materials was obtained in their work through the mathematical derivation: 


$$
\frac{K_{c} \Phi}{H \sqrt{a_{c}}}=0.15 k\left(\frac{c}{a_{c}}\right)^{-3 / 2}
$$

where $\Phi$ is a constraint factor $(\Phi \approx 3), H$ is hardness, $a_{c}$ is half diagonal of indentation impression, $c$ is radial crack length and $k$ is the correction factor for free surface. The value of the correction factor was determined experimentally and was equal to 3.2 for large $c / a_{c}$ values. By taking Vickers hardness relation into consideration $\left(H=0.47 P / a_{c}^{2}\right)$, the expression is rearranged as:

$$
K_{c}=0.0752\left(\frac{P}{c^{3 / 2}}\right)
$$

Based on Evans' work [28], Lawn, Evans and Marshall [15] developed the following fracture toughness model for brittle bulk materials by combining spherical cavity theory. A residual component, $\chi_{r}$, which is determined by the indenter geometries and tested material properties was introduced into the fracture toughness model in their work:

$$
K_{c}=\chi_{r}\left(\frac{P}{c^{3 / 2}}\right)
$$

where $P$ is applied load and $\chi_{r}$ is given by:

$$
\chi_{r}=\xi_{r}^{R}\left(\frac{E}{H}\right)^{1 / 2}(\cot \psi)^{3 / 2}
$$

where $\xi_{r}^{R}$ is a indenter geometry-dependent coefficient and $\Psi$ is the half-angle of the indenter. Meanwhile, the ratio of $E / H$ was replaced by the ratio of $h / a_{c}$ based on the spherical cavity theory, where $h$ is the radius of the plastic zone. The relationship between $E / H$ and $h / a_{c}$ is given by: 


$$
\frac{h}{a_{c}}=\left(\frac{E_{c}}{H_{c}}\right)^{1 / 2} \cdot(\cot \psi)^{1 / 3}
$$

The ratio of $H a_{c}^{2} / P$ determined in Lawn's work is 0.5 , which is similar to the result obtained by Evans and Charles (0.47), and the model can be changed into another form:

$$
\left(\frac{K_{c}}{H \sqrt{a_{c}}}\right)\left(\frac{H}{E}\right)^{1 / 2}=0.028\left(\frac{c}{a_{c}}\right)^{-3 / 2}
$$

The constant 0.028 in this equation was fitted to the experimental fracture toughness data of numerous materials and showed good agreement. However, Eq. (2-18) was developed for evaluating the fracture toughness of brittle bulk materials only.

Based on Lawn's work, Emiliani [29] assessed the fracture toughness of $\mathrm{Y}_{2} \mathrm{O}_{3}$ coating deposited on $\mathrm{Al}_{2} \mathrm{O}_{3}$ and $\mathrm{Nb}$ substrates via Vickers indentation tests, and the results were quite similar to that of bulk $\mathrm{Y}_{2} \mathrm{O}_{3}$ material. Similar to Emiliani's work, several researchers agreed that this model can be applied to brittle coatings under specific situations [30,31], and the refined form of Eq. (2-18) was given by Marshall and Evans [32]:

$$
K_{c}=0.02 E^{1 / 2} P^{1 / 2} a_{c}^{-1 / 2}\left(\frac{c}{a_{c}}\right)^{-3 / 2}
$$

Anstis et al. [13] used a new factor $\xi_{V}^{R}$ to replace the $\xi_{r}^{R} \cot (\psi)^{3 / 2}$ in Eq. (2-20) in order to describe the fully developed radial cracks created by Vickers indenter:

$$
\chi_{r}=\xi_{V}^{R}\left(\frac{E}{H}\right)^{\frac{1}{2}}
$$


In Anstis' work, the parameter $\xi_{V}^{R} 0.016 \pm 0.004$ for fully developed half-penny cracks, which requires that the radial crack length, c, is two times longer than the half indentation diagonal, $a_{c}$.

Although Eq. (2-19) and the parameter $\xi_{V}^{R}=0.016 \pm 0.004$ have been widely applied to fracture toughness evaluation, there are still some problems that need to be solved, such as the pre-existing residual stresses due to the mismatch between different materials and processing methods.

In order to take the effect of residual compressive stress, $\sigma_{r}$, into account, Marshall and Lawn [33] developed an indentation fracture model for well-developed median cracks, whose length is much greater than the radius of plastic zone. The residual stress, $\sigma_{r}$, was successfully added into the fracture toughness model:

$$
K_{c}=\chi_{r}\left(\frac{P}{c^{3 / 2}}\right)-2 m \sigma_{r}\left(\frac{d}{\pi}\right)^{1 / 2}
$$

where $d$ is the depth of median crack and the value of the dimensionless modification factor $m$ is 1 in their work.

Malzbende et al. [34] used this model to evaluate the fracture toughness of the methyltrimethoxysilane (MTMS) and colloidal silica combined coating on float glass substrate. By applying different loads on the coating with the thickness ranging from 0.5 to $4 \mu \mathrm{m}$. Malzbende et al. achieved the conclusion that the composite hardness of the 
coating/substrate system did not change with the applied load when the thickness of coating reached more than $2 \mu \mathrm{m}$. This means that the effect of substrate can be ignored when the coating is thick enough. In this case, the radial cracks could be considered to be totally confined within the surface of coating. Based on this assumption, they argued that Eq. (2-21) was valid for assessing the fracture toughness of the coatings thicker than $2 \mu \mathrm{m}$.

To take the effect of substrate into consideration, Broek [35] refined Eq. (2-21) by introducing a shape factor $Z$ into the formula:

$$
\begin{gathered}
K_{c}=\chi_{r}\left(\frac{P}{c^{3 / 2}}\right)+Z \sigma_{r} c^{1 / 2} \\
Z=1.12 \sqrt{\pi} \frac{d / c}{(3 \pi / 8)+(\pi / 8)(d / c)^{2}}
\end{gathered}
$$

where $Z$ is equal to 1.26 for idealized half-penny crack (the crack length equals to the crack depth).

Based on different crack geometries and material properties, many models have been applied to fracture toughness estimation through indentation tests. Meanwhile, various parameters are also employed to incorporate the crack shape factor into the formulations.

\subsubsection{Fracture toughness models based on energy change}

Irwin first described the relationship between fracture toughness and energy release rate, as demonstrated by Eq. (2-13), using virtual work method and Griffith's fracture theory [26]. 
Based on Irwin's work, Li et al. [36,37] argued that the lengths of radial cracks in a ultra-thin coating were difficult to measure when the thin coating was subjected to a very low load. In order to solve this problem, a load-displacement method which is based on the loading cycle was developed in their research. This method can evaluate the fracture toughness of a thin coating through measuring the profiles of through-thickness cracks. There are three stages for the crack formation process in their theory: (1) first ring-like through-thickness crack formation, (2) delamination and buckling, (3) second ring-like through-thickness crack formation and spalling. This description was used to develop a new method to estimate the strain energy release rate during crack initiation and propagation. As shown in Figure 2-7, the OAC part on the curve stands for the process of first and second ring-like through-thickness crack formation and the area OACE represents the total elastic-plastic energy stored in the coating/substrate system after crack formation. Meanwhile, OAB shows the load-displacement curve without crack formation and the area OBE represents the total elastic-plastic energy stored in the coating/substrate system before crack formation. Based on this depiction, the area $\mathrm{ABC}$ can be used to represent the total released strain energy which leads to the first and second ring-like through-thickness crack formation. Therefore, the strain energy release rate in terms of the strain energy released per unit area of newly created crack surface can be applied to the fracture toughness estimation of coating/substrate systems. 


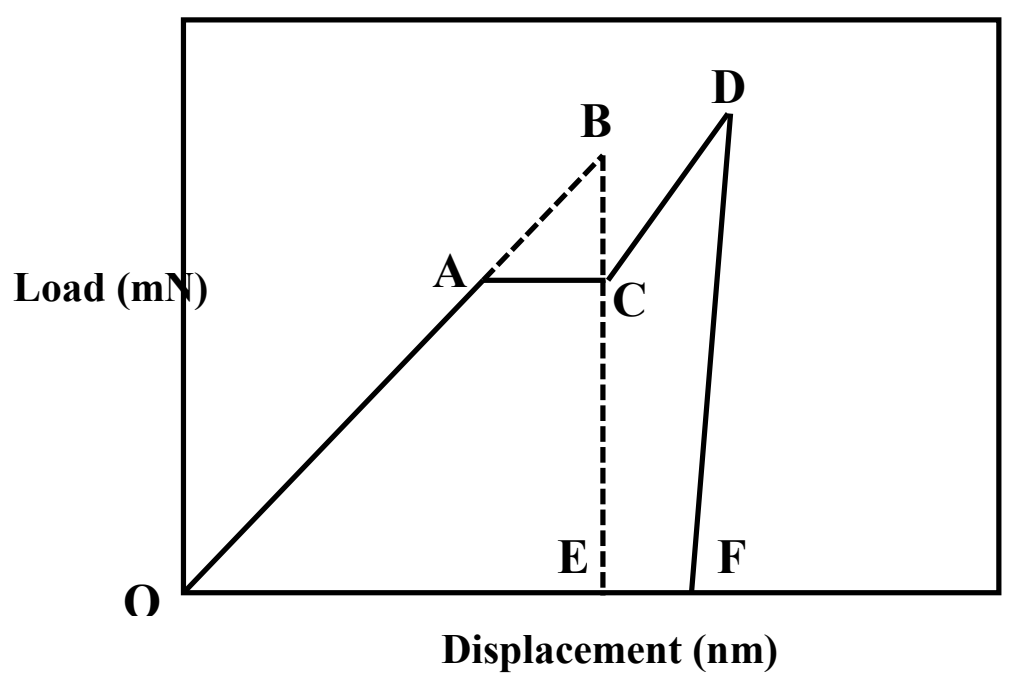

Figure 2-7. Load-displacement curve of indentation test.

In the expression of strain energy release rate, $G$, :

$$
G=\left(\frac{1}{2 \pi C_{R}}\right)\left(\frac{\mathrm{d} U}{\mathrm{~d} C}\right)
$$

the ratio of $\mathrm{d} U / \mathrm{d} C$ was substituted by $U / t$ in their work, where $U$ is the strain energy change during the crack propagation, $C$ is the width of cracks, $2 \pi C_{R}$ is the length of ring-like cracks, and $t$ is the coating thickness. Then Eq. (2-13) is changed into:

$$
K_{I c}=\left[\left(\frac{E}{\left(1-v^{2}\right) 2 \pi C_{R}}\right)\left(\frac{U}{t}\right)\right]^{1 / 2}
$$

Toonder et al. [38] used the energy dissipation during chipping process rather than radial cracks to measure the fracture toughness of thin films. The fracture energy $\Gamma$ was deduced from energy dissipation of chipping behavior $U_{f r}^{c}$ in Toonder's work:

$$
\Gamma=\frac{U_{f r}^{c}}{N \pi t^{\prime} C_{d}}
$$


In Eq. (2-25), $N$ is the total amount of chipped areas, where decohesion occurs and coating is removed from substrate, and $N$ usually equals to 3 in Berkovich indentation tests, $C_{d}$ is the diameter of ring-like cracks, and $t^{\prime}$ is the effective coating thickness which is obtained from the average angle of the chipping edge. Therefore, the fracture toughness of coating/substrate systems is expressed as:

$$
K_{c}=\left[\left(\frac{E}{\left(1-v^{2}\right) N \pi C_{R}}\right)\left(\frac{U_{f r}^{c}}{t}\right)\right]^{1 / 2}
$$

\subsubsection{Fracture toughness models based on stress analysis}

For a brittle coating deposited on a ductile substrate, Hu and Evans [9] proposed that the strain energy release rate, $G_{s s}$, for crack propagation was governed by coating thickness $t$ rather than crack length when the crack length was much greater than the coating thickness. Furthermore, they suggested that for steady-state cracks occurring in coatings, the total strain energy change, $\Delta U_{e}$, can be determined by the coating thickness but not by the crack profiles. Then the strain energy release rate, $G_{s s}$, can be derived from the total strain energy change, $\Delta U_{e}$, and is given by:

$$
\begin{gathered}
G_{s s}=\frac{\Delta U_{e}}{t} \\
\Delta U_{e}=\frac{\pi F\left(\sum\right) \sigma^{2} t^{2}}{E^{c}}
\end{gathered}
$$

where $\Sigma$ is the elastic modulus ratio with $\Sigma=E^{c} / E^{s}, E^{c}$ and $E^{s}$ are the Young's modulus of the coating and substrate, respectively. $F(\Sigma)$ is a function of the elastic modulus ratio $\Sigma$ and is defined as [6], 


$$
F(\Sigma)=\int_{0}^{1} \alpha g(\alpha, \Sigma) \mathrm{d} \alpha
$$

where $\mathrm{g}(\alpha, \Sigma)$ is a function of the ratio of crack depth, $d$, to coating thickness, $t$, and $\alpha=d / t$. The total stress $\sigma$ is defined as:

$$
\sigma=\sigma_{0}+\sigma_{\infty} \frac{E^{c}}{E^{s}}
$$

where $\sigma_{\infty}$ is the applied tension and $\sigma_{0}$ is the residual stress in the system. Finally, the stress intensity factor of the coating/substrate system is described as:

$$
K_{s s}=\sqrt{G_{s s} E^{c}}=\sigma \sqrt{\pi t F\left(\sum\right)}
$$

To evaluate the fracture toughness of mode I crack propagation, another dimensionless parameter in terms of critical cracking number, $\Omega_{c}$, was discussed in the work of $\mathrm{Hu}$ and Evans [9]:

$$
\Omega_{c}=\frac{K_{s s}}{\sigma \sqrt{t}}=\sqrt{\pi F\left(\sum\right)}
$$

For through-thickness cracks, the interface sliding of the area between the coating and the substrate has to be considered. As shown in Figure 2-8, the coating whose thickness is much smaller than that of substrates suffers a constant shear stress, $\tau$, in the interface sliding region. The shear stress, $\tau$, can be determined by the yield strength of the substrate, and this assumption is valid when either sliding or yielding happens to the coating near the interface [9]. 


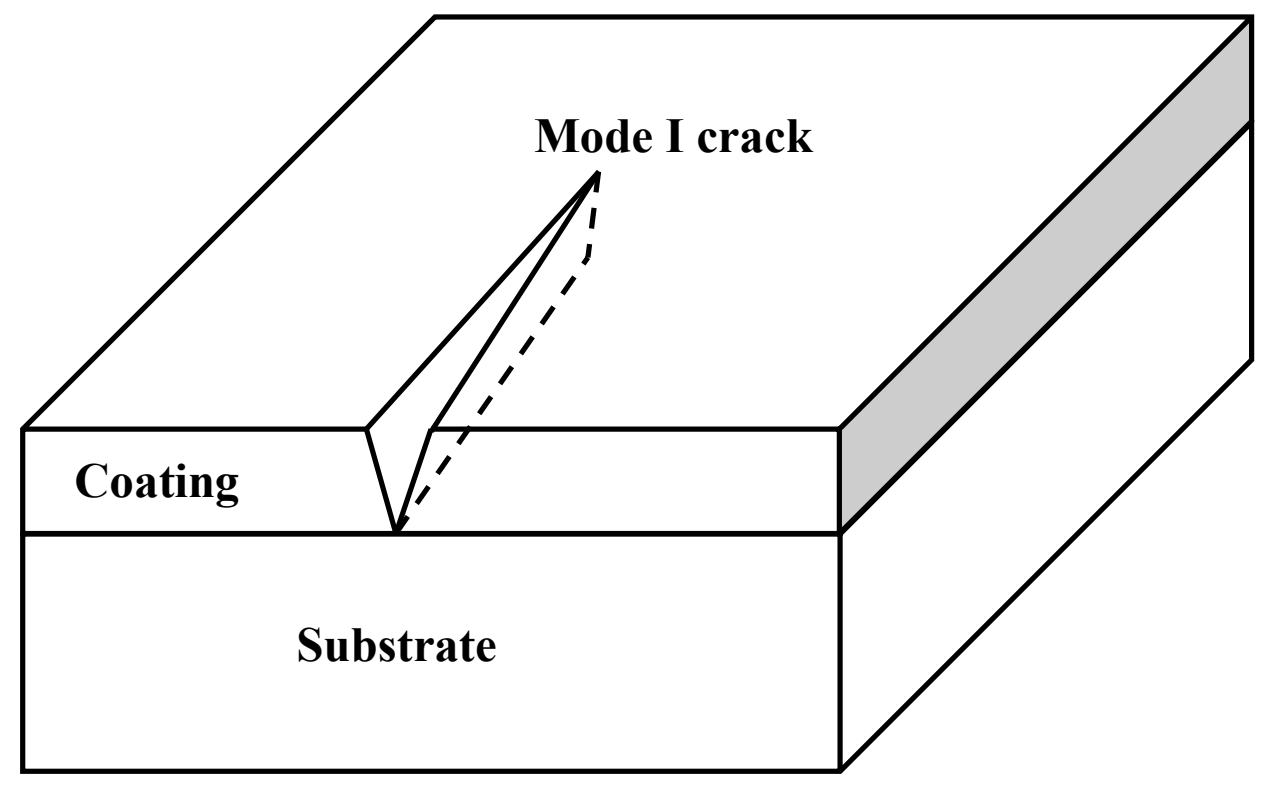

(a)

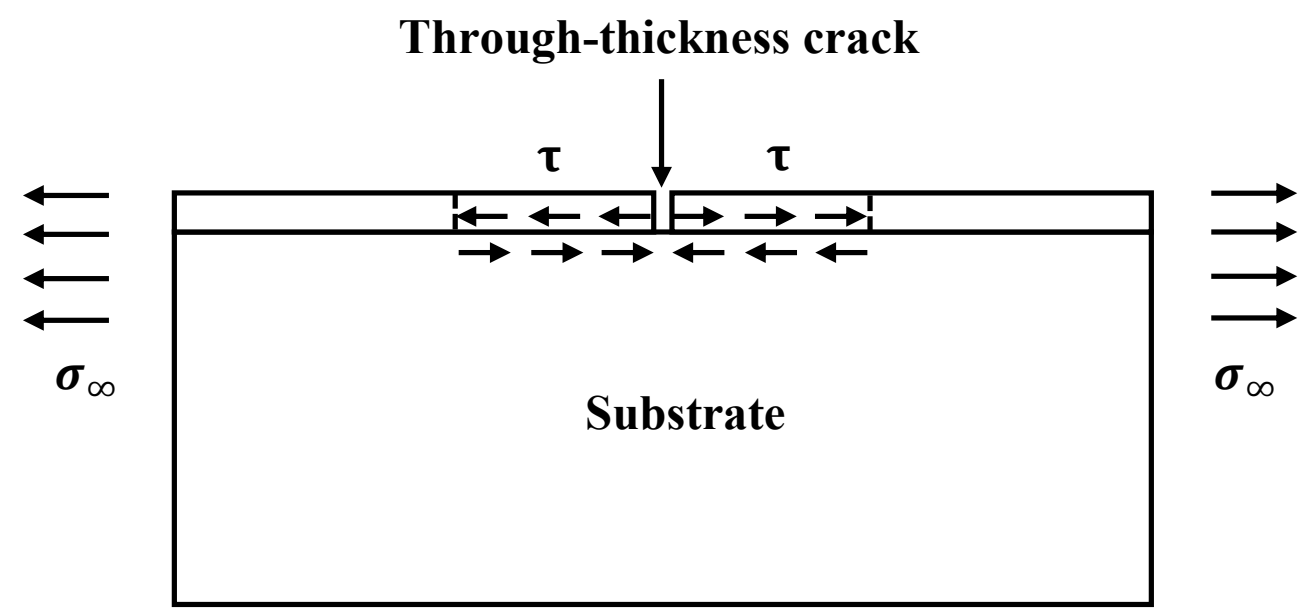

(b)

Figure 2-8. Mode I crack in a coating/substrate system: (a) crack configuration, (b) normal and shear stresses. 
Therefore, the total energy change $\Delta U$ should include the terms caused by the interface sliding and is described as:

$$
\Delta U=\Delta W+\Delta W_{d}+\Delta U_{s}+\Delta U_{e}
$$

where $\Delta W$ is the total work done by the applied load, $\Delta W_{d}$ is the dissipated energy caused by interface sliding, $\Delta U_{s}$ is the strain energy variation due to interface sliding, and $\Delta U_{e}$ is the energy change for steady-state cracking. These terms were derived in the works of Evans [39] and Marshall [40] based on fracture mechanics, and the strain energy release rate is expressed as:

$$
G_{s s}=-\frac{\Delta U}{t}=\frac{\sigma^{2} t}{E^{c}}\left(\frac{\sigma}{3 \tau}+\pi F(\Sigma)\right)
$$

and the stress intensity factor of the coating/substrate system is given by:

$$
K_{s s}=\left[\sigma^{2} t\left(\frac{\sigma}{\sqrt{3} Y}+\pi F\left(\sum\right)\right)\right]^{1 / 2}
$$

where $Y$ is the yield strength of the substrate and $Y=\sqrt{3} \tau$ based on Von Mises yield criterion [41], and the critical cracking number $\Omega_{c}$ is changed into:

$$
\Omega_{c}=\left[\frac{\sigma}{\sqrt{3} Y}+\pi F\left(\sum\right)\right]^{1 / 2}
$$

$\mathrm{Hu}$ and Evans [9] evaluated the fracture toughness of $\mathrm{Cr}$ coating/Al substrate system and $\mathrm{Cr}$ coating/stainless steel substrate system under tensile loading using Eq. (2-34). In the Cr/Al system, the crack linear density and the critical fracture stress decreased with the increase in coating thickness. For Cr/stainless steel system, the critical fracture stress was higher than that of the $\mathrm{Cr} / \mathrm{Al}$ system due to the difference of yield tensile stresses in the two substrates. However, the calculated fracture toughness values of these two systems were similar 
$\left(2.8 \pm 0.3 \mathrm{MPa} \cdot \mathrm{m}^{1 / 2}\right.$ for $\mathrm{Cr} / \mathrm{Al}$ and $2.6 \pm 0.4 \mathrm{MPa} \cdot \mathrm{m}^{1 / 2}$ for $\mathrm{Cr} /$ stainless steel), which validated the models developed in their work.

Harry et al. [42] estimated the fracture toughness of pure $\mathrm{W}$ coating and WC coating deposited on stainless steel substrate via three-point-bending tests and micro-tensile tests. The thickness of $\mathrm{W}$ coating specimens varied from 2 to $16.4 \mu \mathrm{m}$, and the calculated fracture toughness for the coating ranged from $1.01 \pm 0.3 \mathrm{MPa} \cdot \mathrm{m}^{1 / 2}$ to $2.4 \pm 0.5 \mathrm{MPa} \cdot \mathrm{m}^{1 / 2}$ via Eq. (2-34). Similarly, the thickness of WC coating changed from 1.8 to $16 \mu \mathrm{m}$, and the calculated fracture toughness for the coating increased from $0.28 \pm 0.2 \mathrm{MPa} \cdot \mathrm{m}^{1 / 2}$ to $1 \pm 0.2 \mathrm{MPa} \cdot \mathrm{m}^{1 / 2}$. For both types of coatings, the specimens with thicker coating had higher fracture toughness. These results were consistent with the assumptions that the critical fracture stress, critical fracture strain energy release rate and the fracture toughness for both coating/substrate systems were obviously lower than those of bulk materials with the same chemical compositions. Eq. (2-34) was also used to investigate the fracture toughness of multilayer WC-based coatings $[43,44]$. It showed that the specimens with more layers and thicker coating had better fracture resistance.

Dalgleish et al. [45] assessed the fracture behavior of $\mathrm{Al}_{2} \mathrm{O}_{3} / \mathrm{Al}$ lamintes and $\mathrm{Al}_{2} \mathrm{O}_{3} / \mathrm{Al}-\mathrm{Mg}$ laminates. The out-layer $\mathrm{Al}_{2} \mathrm{O}_{3}$ was treated as the brittle coating and the mid-layer aluminum alloys were regarded as ductile substrate in their work. The fracture toughness of the laminates was calculated using Eq. (2-34) which was $3 \mathrm{MPa} \cdot \mathrm{m}^{1 / 2}$ for both types of 
specimens, and the critical value of the dimensionless parameter $\Omega_{c}$ was estimated to be $1 \pm 0.1$ for the specimens. For steady-state cracks, as demonstrated in their work, the fracture toughness of the laminates specimens which consist of a hard outer-layer and a soft mid-layer was similar to that of the brittle coating/ductile substrate systems having the same chemical compositions.

Wang et al. [46] investigated the fracture toughness of thin diamond-like carbon (DLC) coating deposited on a steel substrate using Hu's model. The steel substrate was subjected to tension loading in the tests. With the deformation of substrate, neither decohesion or spalling behavior occurred in the coating/substrate system, and the main damage mechanism of the coating was steady-state cracking. The results showed that the fracture toughness of DLC coating/steel substrate system was $2 \mathrm{MPa} \cdot \mathrm{m}^{1 / 2}$.

Eq. (2-34) has also been applied to evaluating the fracture toughness of titanium nitride/vanadium nitride alloy coating deposited on stainless steel by Latella et al. [47]. In their work, the total stress, $\sigma$, was replaced by $\sigma_{c}$ in terms of fracture strength or critical stress, and had an expression:

$$
\sigma_{c}=\varepsilon_{\exp } E^{c}+\varepsilon_{r} E^{c}
$$

where $\varepsilon_{\text {exp }}$ is the strain measured in the first cracking process during tensile tests and $\varepsilon_{r}$ is the residual strain existing in the coating. Thus, the fracture toughness for coating/substrate system was expressed as: 


$$
K_{I c}=\left[\sigma_{c}^{2} t\left(\frac{\sigma_{c}}{\sqrt{3} \sigma_{Y}}+\pi F(\Sigma)\right)\right]^{1 / 2}
$$

where $\sigma_{Y}$ is the yield stress of substrate materials.

\subsubsection{Temperature-dependent fracture toughness models at macroscopic}

\section{level}

Fracture toughness data at high temperatures have been rarely reported, since only limited temperature-dependent fracture toughness tests and relevant researches have been conducted on materials. Compared with the fracture toughness models based on measurements and experiments at room temperature, the analytical simulation for temperature-dependent fracture toughness of materials has been seldom developed.

Somerday et al. [48] derived a formula to evaluate the fracture toughness of materials at elevated temperatures for small scale yielding situations:

$$
K_{I c}=\sqrt{\frac{\sigma_{c s} E \delta}{\left(1-v^{2}\right) d_{n}}}
$$

where $\sigma_{c s}$ is the critical stress and $E$ is Young's modulus, $d_{n}$ is a dimensionless constant that is determined by strain-hardening exponent $n$, stress state, $\sigma_{y s} / E$, and Mode I crack tip opening displacement $\delta$.

This equation was applied and slightly modified by Haynes et al. [49,50] to evaluate the high-temperature fracture toughness of aluminum alloys. 


$$
K_{I c}=\sqrt{\frac{\sigma_{y s} E l^{*} \varepsilon_{f}^{*}}{\left(1-v^{2}\right) d_{n}}}
$$

where $l^{*}$ and $\varepsilon_{f}^{*}$ are critical distance ahead the crack tip and critical fracture strain, respectively. In their work, Eq. (2-39) cannot be used to build the relationship between temperature and fracture toughness, $K_{I c}$, directly. However, it can be applied to predicting the effect of temperature on the fracture behavior of such materials. In this equation, $\sigma_{y s}, E$, $\varepsilon_{f}^{*}$ and $d_{n}$ are assumed to vary with temperature. The fracture toughness of several types of aluminum alloy was successfully predicted and compared with the experimental data in their work.

Similar to that in Haynes's research, the models used in other researches [51-53] to evaluate the temperature-dependent fracture toughness of materials concerned some parameters or material properties such as Young's modulus or critical stress, which were subjected to temperature shifts. However, these models are only valid for specific materials under certain circumstances, and most of them were derived from test data or empirical equations.

\subsection{Fracture Toughness Models at Microscopic Level}

It was noted that the critical stress obtained from Eq. (2-40) was much greater than the test results, Griffith suggested that the pre-existing defects in materials had a remarkable effect on the strength of materials, and this effect should be taken into consideration [54].

$$
\sigma_{c s f} \cong \frac{E}{10}
$$


Based on Griffith's energy balance criterion, the total energy transition during the crack propagation process in terms of the total amount of surface energy change should be equal to the sum of work done by applied load and the reduced elastic energy storage, since new surfaces would be created in this process.

For a straight through-thickness crack with length $2 a$ in an infinite plate, as shown in Figure 2-2, Inglis [55] derived that the elastic energy change is:

$$
-\Delta U_{e}=\frac{\pi \sigma^{2} a^{2}}{E}
$$

and the surface energy is:

$$
\Delta U_{s}=4 \gamma a
$$

Then the total energy change and critical stress can be determined from the following equations:

$$
\begin{gathered}
\Delta U_{s}=\Delta U_{\text {total }}+\left(-\Delta U_{e}\right) \\
\frac{\mathrm{d} \Delta U_{\text {total }}}{\mathrm{d} a}=\frac{\mathrm{d}\left(-\pi \sigma^{2} a^{2} / E+4 \gamma a\right)}{\mathrm{d} a}=0
\end{gathered}
$$

As introduced in previous sections, most of the existing fracture toughness models were derived from Griffith's theory or based on tests and measurements. However, these models do not take critical factors, for example, temperature, into the fracture toughness estimation. In order to solve this problem, temperature-dependent models based on micromechanics and thermodynamics are needed to develop fracture-toughness-prediction of materials at high temperatures. 


\subsubsection{The Arrhenius-type equation and rate controlling theory}

In terms of the constitutive laws, the total amount of energy change and the dislocation movement of atoms in materials during the fracture process can be used to describe the crack initiation and extension behaviors [56].

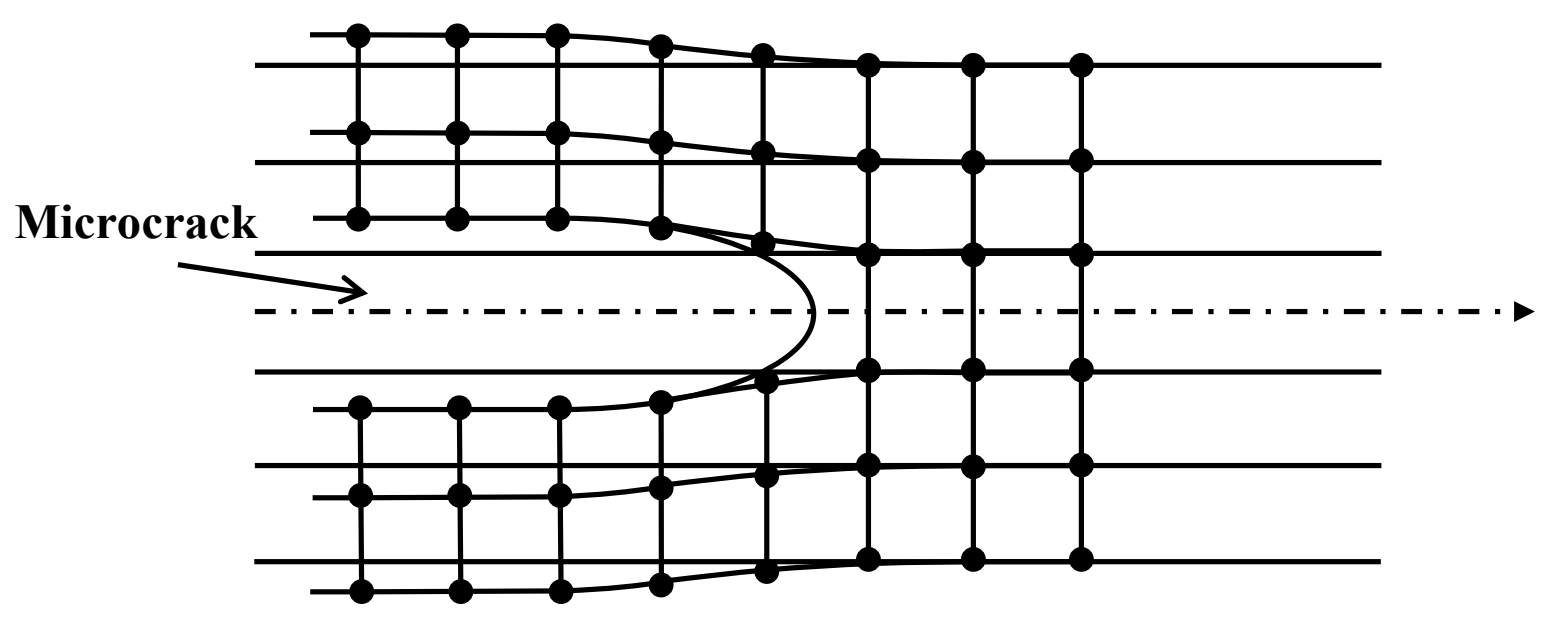

Figure 2-9. Microcrack formation and the movement of atoms.

The total energy change during the crack propagation process can be described using strain energy release rate, surface energy, barrier energy and stacking fault energy. The dislocation movement of atoms in this process can be depicted by Burgers vector and slip system of the material under certain type of load. As shown in Figure 2-9, the microcracks generated under tensile stress can be described by the movement of atoms. When deformation occurs in materials, the atoms in certain area may deviate from their equilibrium position. During the atomic deviation process, energy change takes place in the bonds between atoms, the bonds are broken when the force or the energy exceeds a critical value. Microcracks are initiated 
and propagate due to the atomic deviation and the absence of connection between atoms. Although this description is based on a 2D profile and much simpler than real situation, it can be applied to the analysis of microcrack formation and the fracture toughness model at micromechanics scale.

The fracture behavior and the plastic deformation of materials can be treated as a thermally activated process and be described by the Arrhenius-type equation [57]:

$$
\dot{\gamma}=A \exp \left(-\frac{\Delta E}{k T}\right)
$$

where $\dot{\gamma}$ is a general rate of the thermally activated process, $\Delta E$ is general energy change, $A$ is a pre-exponential factor, $T$ is absolute temperature and $k$ is Boltzmann constant.

Based on the Arrhenius-equation, Krausz and Eyring [58] described the plastic deformation using the kinetics of chemical reactions, $\dot{s}=b \rho v_{d}$, where $\dot{s}$ is the strain rate during plastic deformation, $b$ is Burgers vector in terms the magnitude of dislocation movement of atoms, $\rho$ is dislocation density and $v_{d}$ is dislocation velocity. According to this description, they derived a temperature-dependent rate constant, $s$, to describe the strain rate in a plastic deformation area:

$$
s=v \exp \left(-\frac{\Delta G(W)}{k T}\right)
$$

where $v$ is the frequency factor and $\Delta G(W)$ is the thermal energy which contributes to plastic deformation. 
The thermal energy $\Delta G(W)$ can also be described as the difference between bond energy, $\Delta G$, and mechanical energy, $W(\sigma)$, in terms of work done by applied stress. Then Eq. (2-44) is then rewritten as:

$$
s=v \exp \left(-\frac{\Delta G-W(\sigma)}{k T}\right)
$$

Eq. (2-45) was also defined as a kinetics equation. The frequency factor $v$ was afterwards refined in Krausz's work [59] and Eq. (2-45) can be expressed as:

$$
s=\frac{k T}{h} \exp \left(-\frac{\Delta G-W(\sigma)}{k T}\right)
$$

where $h$ is the Planck's constant.

Based on this equation, the crack velocity, $v$, is expressed as:

$$
v=n a_{0} s
$$

where $n$ stands for the total amount of interatomic distance, $a_{0}$, which is used to measure the length of crack extension.

Similar to the kinetics equation, the state equation describes the continuous variation in microstructure during the thermally activated process in terms of plastic deformation [60]. In the state equation, the work done by applied stress, $W(\sigma)$, is replaced by the product of activation volume, $V$, and effective stress, $\sigma$, which is equal to the difference between applied stress, $\sigma_{a p p}$, and internal stress, $\sigma_{\text {int }}[61]$. Then the strain rate is given by: 


$$
\frac{\partial \varepsilon}{\partial t}=\rho \varepsilon^{0} v \exp \left(-\frac{\Delta G-V\left(\sigma_{a p p}-\sigma_{\text {int }}\right)}{k T}\right)
$$

where $\rho$ is dislocation density, $\varepsilon^{0}$ is the contribution of thermal activation to the strain.

Tyson and Conrad [62] studied the plastic deformation behavior of hafnium oxygen alloys. They estimated the activation energies of the specimens using the tensile test data obtained at different temperatures (from 77 to $750 \mathrm{~K}$ ), and pointed out that the free activation energy should be divided into two categories based on whether the internal barrier force for plastic deformation is temperature dependent. The activation energy can be expressed as follows if the internal force does not change at elevated temperatures:

$$
\Delta G^{0}=\Delta H^{*}=Q^{*}+\frac{1}{2} \tau^{*} v \alpha
$$

where $\Delta H^{*}$ is activation enthalpy and $Q^{*}, v$ and $\alpha$ are expressed as:

$$
\begin{gathered}
Q^{*}=-T v\left(\frac{\partial \tau^{*}}{\partial T}\right)_{\dot{\gamma}} \\
v=k T\left(\frac{\partial \tau^{*}}{\partial \dot{\gamma}}\right)_{T} \\
\alpha=-\frac{T}{\mu} \frac{\mathrm{d} \mu}{\mathrm{d} T}
\end{gathered}
$$

When the internal barrier force is temperature dependent, the activation energy is given by:

$$
\Delta G^{T}=\frac{Q^{*}-\tau^{*} v \alpha}{1+\alpha}
$$

Using the crack velocity equation based on the rate theory, Atkinson [63] studied the fracture behavior of synthetic quartz specimen via double torsion tests, and built the relationship 
between the stress intensity factor, $K_{I}$, and crack growth rate, $v_{c}$. The crack velocity equation is expressed as:

$$
v_{c}=v_{0} K_{I}^{n} \exp \left(-\frac{\Delta H}{R T}\right)
$$

where $\Delta H$ is activation enthalpy, $v_{0}$ is frequency related factor, $n$ is stress corrosion index which can be determined from experiments, $R$ is the gas constant.

Raj and Farmer [64] evaluated the creep resistance and analyzed the dislocation pattern of polycrystalline NiAl using power-law relation and the rate theory. The temperature-dependent strain rate equation is written as:

$$
\frac{\partial \varepsilon}{\partial t}=A\left(\frac{E}{T}\right)\left(\frac{\sigma}{E}\right)^{n} \exp \left(-\frac{Q_{c}}{R T}\right)
$$

where $A$ is pre-exponential factor decided by the Burgers vector, Young's modulus, Boltzmann's constant and grain size of $\mathrm{NiAl}, R$ is the gas constant, $\sigma$ is applied stress and $Q_{c}$ is activation energy.

Yin et al. [65] used the Arrhenius-type equation to assess the temperature-dependent plastic deformation of Ti-50A. The strain rate is given by:

$$
\dot{\varepsilon}=\dot{\varepsilon}_{0} \exp \left(-\frac{\Delta G(\sigma, T)}{k T}\right)
$$

where

$$
\dot{\varepsilon}_{0}=\left(\frac{1}{\bar{M}}\right)\left(\frac{\rho_{m}}{l^{*}}\right)(A b)\left(\frac{b}{l^{*}} v_{D}\right)
$$


and the free activation energy was derived as:

$$
\Delta G=k T \ln \left(\frac{\dot{\varepsilon}_{0}}{\dot{\varepsilon}}\right)
$$

where $\rho_{m}$ is mobile dislocation density, $l^{*}$ is the length of dislocation line, $A$ is the area of slip plane and $v_{D}$ is Debye frequency. $\bar{M}$ is the Taylor orientation factor which builds the relationship between stress and strain in tensile direction and shear direction. The length of dislocation line, $l^{*}$, has the following expression based on the Freidel relation:

$$
l^{*}=\left(\frac{2 \bar{M} E_{L}}{N \sigma^{*} b}\right)^{1 / 3}
$$

where $\sigma^{*}$ is the tensile stress caused by thermal activation, $N$ is obstacle density and $E_{L}$ is the tension on dislocation line.

The free activation energy in Yin's work has the same form as in the work of Tyson and Conrad [62], and the activation volume is also derived and expressed as:

$$
v^{*}=-\left(\frac{\partial \Delta G}{\partial \tau^{*}}\right)_{T}=b l^{*} x^{*}
$$

where $x^{*}$ is the thermal activation distance. The pre-exponential factors $\bar{M}, A, v_{D}$ are given as $\bar{M}=2.5, A=l^{* 2}$ and $v_{D}=10^{13} s^{-1}$. The parameters obtained from the equations discussed above successfully described the deformation behavior of Ti-50A in the work of Yin et al. [65], and those results were consistent with results reported in other research on polycrystalline titanium materials. Some critical parameters, such as activation volume, $v^{*}$, were applied to depicting the dislocation movement of the microstructure. 
Conrad [66] derived plastic deformation equations for $\mathrm{Cu}$ in three regimes with different grain sizes based on the rate controlling theory, and the results were calculated over a temperature range from 77 to $373 \mathrm{~K}$. For the first regime $\left(d \approx 10^{-3} \sim 10^{-6} \mathrm{~m}\right)$, the strain rate is described as:

$$
\dot{\gamma}=\dot{\gamma}_{0} \exp \left(-\frac{\Delta G\left(\tau_{e}^{*}\right)}{k T}\right)
$$

where

$$
\dot{\gamma}_{0}=\left(\frac{\rho_{m}}{l^{*}}\right)\left(l^{*}\right)^{2} b\left(\frac{b}{l^{*}} v_{D}\right)=\rho_{m} b^{2} v_{D}
$$

and $\tau_{e}^{*}$ is effective shear stress which is equal to $\left(\tau-\tau_{\mu}\right), \tau$ is applied shear stress and $\tau_{\mu}$ is intrinsic barrier shear stress. The Debye frequency, $v_{D}$, is also equal to $10^{13} \mathrm{~s}^{-1}$ in this work, and mobile dislocation density, $\rho_{m} \approx(0.1-0.5) \rho$, where $\rho$ is the total dislocation density,

$$
\rho=\left(\frac{\tau}{\alpha \mu b}\right)^{2}
$$

The relationship between resolved shear stress $\tau_{b}$ and dislocation density $\rho_{b}$ is described as:

$$
\tau_{b}=\alpha \mu b \rho_{b}^{1 / 2}
$$

where $\alpha \approx 0.5$ for most metals [67], $\mu$ is shear modulus and

$$
\rho=\frac{\beta \gamma}{b g}
$$

where $\beta$ is a constant and ranges from 0.25 to $2, \gamma$ is resolved shear strain and $g$ is grain size. 
The Gibbs free activation energy in Eq. (2-57a) has the same form as in Eq. (2-51) due to the effect of shear stress, $\tau$, which is given by:

$$
\tau=\frac{\mu b}{2 \pi l_{d}}
$$

for screw dislocation, and

$$
\tau=\frac{\mu b}{2 \pi(1-v) l_{d}}
$$

for edge dislocation, where $l_{d}$ is the distance between dislocation lines. The activation volume, $v^{*}$, in Conrad's work is described as:

$$
v^{*}=-\left(\frac{\partial \Delta G}{\partial \tau^{*}}\right)_{T}=b l^{*} x^{*}=\left(\frac{3}{2} v^{a}\right)
$$

where $v^{a}$ is apparent activation volume,

$$
v^{a}=M k T \frac{\partial \ln \varepsilon}{\partial \sigma}
$$

and $M$ is the Taylor orientation factor, which is equal to $\sqrt{3}$.

The dislocation behavior of $\mathrm{Cu}$ was quite different in other regimes with smaller grain size $\left(d<10^{-6} \mathrm{~m}\right)$, and these differences were caused by the dislocation cell structure and intragranular dislocation activities [66].

Using Eq. (2-57), Wang et al. [68] discussed the effect of temperature on the strain rate sensitivity and the activation volume of nanocrystalline Ni with the grain size about $30 \mathrm{~nm}$ via strain rate jump tests, and the tests were conducted from 77 to $373 \mathrm{~K}$. To obtain the 
activation energy and activation volume, the strain rates of specimens were obtained from the repeated stress relaxation tests, and their results were accordant with the analytical values.

Atkins et al. [69] investigated the temperature-dependent fracture behavior of PMMA using Visicorder circuitry test method. The critical parameters for describing the fracture behavior such as critical fracture load, crack length and crack tip opening displacement, were recorded over a temperature range from 193 to $353 \mathrm{~K}$, and the critical stress intensity factor, $K_{I}$, was obtained directly from the load-displacement curves that were recorded in the tests, according to Gross-Srawley expression, which concerns the dimension and properties of the specimen material. The test data were compared with the analytical results which were estimated from the rate-controlling theory based expression:

$$
v_{c}=A_{1} \exp \left(-\frac{U-\lambda G_{s s}}{k T}\right)
$$

where $v_{c}$ is crack extension velocity, $A_{1}$ and $\lambda$ are constants, $G_{S S}$ is strain energy release rate. It was shown that the rate-controlling theory based expression can estimate the activation energy and crack growth rate over the selected temperature range accurately when compared with the test data.

Phillips et al. [70] performed double torsion tests on amine hardener cured epoxide resin to study its fracture behavior. The relationship between the test temperature and the critical crack energy was obtained experimentally, showing that the crack initiation energy, $G_{i}$, had a substantial increase at elevated temperatures, but the crack arrest energy, $G_{a}$, remained 
constant during this process. Based on the rate controlling equation in Atkins' work [68], Phillips et al. discussed the yield stress, strain rate and activation energy variations of epoxide resin with increasing temperature.

Margolin et al. [71] developed a probabilistic model to evaluate the fracture toughness of 2.5CrMoV nuclear pressure vessel steel. The temperature and yield stress dependent critical parameter $m_{T}$ can be expressed as $m_{T}(T)=m_{0} \sigma_{Y S}(T)$, where $m_{0}$ is a constant determined from experiments, and $\sigma_{Y S}(T)$ is the temperature-dependent component of yield stress. The relationship between $\sigma_{Y S}(T)$ and temperature, $T$, was derived based on the rate controlling theory:

$$
\dot{\varepsilon}=\dot{\varepsilon}_{0} \exp \left(-\frac{U\left(\sigma_{Y S}\right)}{k T}\right)
$$

where $U\left(\sigma_{Y S}\right)$ is the activation energy calculated from $\sigma_{Y S}$, and $\sigma_{Y S}(T)$ can be described using a temperature-dependent model $\sigma_{Y S}(T)=\sigma_{Y S}^{0} \exp (h T)$, where $h$ is a test-based constant.

To verify the accuracy of the estimated yield stress, $\sigma_{Y S}(T)$, the calculated results of the total yield stress, $\sigma_{Y}(T)$, which was expressed as $\sigma_{Y}(T)=\sigma_{Y G}+\sigma_{Y S}(T)$, was compared with the tested data over a temperature range from 77 to $313 \mathrm{~K}$. The results showed that the calculated $\sigma_{Y}(T)$ was in full agreement with that obtained from tests [71]. 
Kawasaki et al. [72] measured the fracture toughness and fatigue crack growth rate of a high strength steel on a temperature and load controlled universal testing machine. The test results were in good accordance with the estimated results from the expression based on the rate controlling theory:

$$
\frac{\mathrm{d} c}{\mathrm{~d} N}=\ln \frac{A}{f^{\lambda}}(\Delta K)^{b} \exp \left(\frac{U-a \ln \Delta K}{k T}\right)
$$

where $A$ and $\lambda$ are test-based constant, $a$ and $b$ are material constants, $f$ is cyclic frequency and $\Delta K$ is the range of stress intensity factor.

Huang et al. [73] reported the numerical simulation for describing the inhomogeneous deformation of metallic glasses. The inhomogeneous deformation problem was solved through analyzing the shear problems in the material by using the rate theory based equation, and the relationship between shear strain rate, $\dot{\gamma}$, and shear stress, $\tau$, is expressed based on the free volume concentration theory:

$$
\dot{\gamma}=2 v_{0} \exp \left[-\frac{\alpha V^{*}}{V_{f}}-\frac{\Delta G}{k T}\right] \sinh \left[\frac{\tau \Omega}{2 k T}\right]
$$

where $v_{0}$ is a frequency related factor, $\alpha$ is a geometry-determined constant, $\Delta G$ is activation energy, $\Omega$ is atomic volume, $V^{*}$ and $V_{f}$ are critical volume and average free volume, respectively. This rate controlling theory based function successfully depicted the inhomogeneous deformation behavior of the metallic glass materials. 
Gumbsch [74] investigated the brittle-to-ductile transition (BDT) and fracture behavior of tungsten crystal based on experimental observations and the rate controlling equation. To examine the effects of loading rate on the fracture behavior and brittle-to-ductile transition time (BDTT), Gumbsch studied the temperature-dependent fracture toughness of pre-deformed tungsten single crystals using previous experimental data, and found the influences of activation energy, $Q$, shear stress, $\tau$, and temperature, $T$, on the dislocation velocity, $v_{d}$, and strain rate, according to the rate controlling equation:

$$
v_{d}=v_{0}\left(\frac{\tau}{\tau_{0}}\right)^{m} \exp \left(-\frac{Q}{k T}\right)
$$

where $v_{0}$ is a pre-exponential constant, $\tau_{0}$ is normalization stress with unit magnitude and determined to be 1MPa in Gumbsch's work.

Using the same rate controlling equation, Zeng and Hartmaier [75] analyzed the effects of grain size and the curvature radius of the crack tip on the dislocation velocity and crack behavior of tungsten poly-crystals. The results showed that the increase in grain size can decrease the influence of embrittlement on the tungsten poly-crystals, and the fracture toughness of the specimens can be enhanced as a result of grain size increase.

Brennan and Prewo [76] studied the fracture toughness and steady-state creep rate of silicon carbide (SiC) fibre reinforced lithium aluminosilicate (LAS) glass-ceramic from room temperature to $1000{ }^{\circ} \mathrm{C}$ in terms of the rate controlling theory and the Arrhenius-type 
equation. Sano and Kudo [77] investigated the crack growth rate and stress intensity factor-crack velocity diagram of granitic rocks based on the same theory.

According to the deformation theory given by Kraus [61], the temperature-dependent strain rate can be expressed as:

$$
\gamma=\gamma_{0} \exp \left(-\frac{\Delta G^{*}[W(\tau)]}{k T}\right)
$$

where $G^{*}[W(\tau)]$ is the Gibbs free activation energy or apparent activation energy, which is given by:

$$
\Delta G^{*}[W(\tau)]=\Delta G^{*}-W(\tau)
$$

where $G^{*}$ is actual activation energy and $W(\tau)$ is the work done by applied shear stress. The pre-exponent factor $\gamma_{0}$ is defined as:

$$
\gamma_{0}=\alpha b \rho v
$$

where $\alpha$ is a transition factor which connects the dislocation velocity with the strain rate, $b$ is Burgers vector, $\rho$ is mobile dislocation density and $v$ is given as $6 \times 10^{-12} s^{-1}$.

The constitutive laws and rate controlling theory successfully take the temperature and microstructure factors into the strain rate and plastic deformation rate calculations. Based on these theories, a microscopic level temperature-dependent model for fracture toughness evaluation has been proposed. 


\subsubsection{Temperature-dependent fracture toughness models at microscopic}

\section{level}

The elastic energy release rate criterion, $G_{c}$, was first defined in Irwin's work as described by Eq. (2-7). The cracks grow when the elastic energy release rate, $G$, exceeds the critical value, $G_{c}$, which is equal to two times of surface energy.

Rice [78] provided another method using the $J$ integral, a path-independent integral, to calculate the strain energy release rate. The strain energy release rate, $G$, in the material subjected to plastic deformation can be expressed as the summation of surface energy and plastic deformation energy. In Rice's work, the critical value of $J$ integral for crack propagation is given by:

$$
J_{c}=J_{s}+J_{d}
$$

where $J_{S}$ is the work done by elastic deformation which equals to $2 \gamma_{s}$, and $\gamma_{s}$ is the surface energy, $J_{d}$ is the plastic dissipation energy for crack propagation and can be described as:

$$
J_{d}=\sigma \varepsilon h
$$

where $\sigma$ is the stress field near the crack tip, $\varepsilon$ is the strain field near the crack tip and $h$ is the dimension of plastic zone.

To incorporate the temperature effect into the fracture toughness estimation, the plastic deformation rate, $\dot{\gamma}$, is described by the Arrhenius-type equation and Gibbs free energy of activation based on the rate controlling theory and thermal activated dislocation theory [79]: 


$$
\dot{\gamma}=\dot{\gamma}_{0} \exp \left(-\frac{\Delta G}{k T}\right)
$$

where $k$ is Boltzmann constant, $T$ is absolute temperature, $\dot{\gamma}_{0}$ is a dimensionless factor which is determined by the density of activation points, $N$, in the process zone, the dislocation area, $A^{*}$, Burgers vector, $b$, and the activation frequency, $v^{\prime}$, in the dislocation area:

$$
\dot{\gamma}_{0}=N A^{*} b v^{\prime}
$$

The Gibbs free energy of activation, $\Delta G$, is given by:

$$
\Delta G=\Delta F-v^{*} \tau
$$

where $v^{*}$ is the activation volume defined as the product of the length of dislocation line, $l$, activation distance, $x^{*}$, and Burgers vector:

$$
v^{*}=b l x^{*}=A^{*} x^{*}
$$

The work done by external work is described as the product of shear stress, $\tau$, and the activation volume, $v^{*}$. By integrating the exponential term over time, the total strain during the deformation process can be written as [14]:

$$
\gamma=\left(\frac{\dot{\gamma}_{0} k T t}{b l x^{*} \tau}\right) \exp \left(-\frac{\Delta F-v^{*} \tau}{k T}\right)
$$

By replacing the terms $\sigma=\sqrt{3} \tau, \gamma=\sqrt{3} \varepsilon$, integrating Eq. (2-70) and (2-73), the plastic dissipation energy, $J_{d}$, can be described as:

$$
\begin{gathered}
J_{d}=J_{0} \exp \left(-\frac{\Delta F-v^{*} \tau}{k T}\right) \\
J_{0}=\frac{\dot{\gamma}_{0} k T t h}{b l x^{*}}
\end{gathered}
$$


The intrinsic energy barrier, $\Delta F$, for dislocation motion can be described as:

$$
\Delta F=\gamma_{u s}+\gamma_{F}+U_{P-N}
$$

where $\gamma_{u s}$ is unstable stacking fault energy, $\gamma_{F}$ is generalized stacking fault energy and $U_{P-N}$ is Peierls-Nabarro $(\mathrm{P}-\mathrm{N})$ energy barrier.

By combining Eq. (2-75) into Eq. (2-74), the plastic energy dissipation, $J_{d}$, can be expressed as:

$$
J_{d}=J_{0} \exp \left[-\frac{A^{*}\left[\left(\gamma_{F}+U_{P-N}\right)+\left(\gamma_{u s}-x^{*} \tau\right)\right]}{k T}\right]
$$

In Eq. (2-76), the unstable stacking fault energy, $\gamma_{u s}$, can be assumed to be equal to the work done by shear stress, $\tau x^{*}$, when the deformation in near crack tip area is initiated. By substituting Burgers vector, $b$, for $x^{*}, b^{2}$ for $A^{*}, b^{3}$ for $v^{*}$, Eq. (2-53) is modified as:

$$
J_{d}=J_{0} \exp \left[-\frac{b^{2}\left(\gamma_{F}+U_{P-N}\right)}{k T}\right]
$$

Meanwhile, the temperature-dependent fracture toughness model for plane strain is depicted as:

$$
K_{l c}=\sqrt{\frac{E\left(J_{s}+J_{d}\right)}{1-v^{2}}}
$$

In Chan's work $[14,80,81]$, the temperature-dependent fracture toughness of several intermetallics and composite materials, such as $\mathrm{NiAl}$ and $\mathrm{TiAl}$, was estimated based on the slip system, stacking fault energy and $\mathrm{P}-\mathrm{N}$ energy barrier of the materials. The calculated results were compared with experimental data and exhibited good consistency. However, some critical parameters such as dislocation density, $\rho$, plastic zone dimension, $h$, and 
pre-exponential factor, $J_{0}$, were assumed to be unchanged due to their inaccessibility. The dislocation density, $\rho$, was estimated as $3 \times 10^{11} 1 / \mathrm{m}^{2}$ for both plane strain and non-plane strain conditions, the plastic zone dimension, $h$, was assumed to be $1 \mathrm{~mm}$ for plane strain condition and $2.5 \mathrm{~mm}$ for non-plane strain condition, the approximate pre-exponential parameter $J_{0}$ was $5 \times 10^{4} \mathrm{~J} / \mathrm{m}^{2}$ for plane strain condition and $3 \times 10^{5} \mathrm{~J} / \mathrm{m}^{2}$ for non-plane strain condition. Although these estimations were based on credible experimental data, the dislocation density, $\rho$, the plastic zone dimension, $h$, and the pre-exponential factor, $J_{0}$, could be different for various types of materials, and these critical parameters should have a significant influence on the calculated fracture toughness. Besides, the fracture toughness model applied in Chan's work was developed for bulk materials. Therefore, this model needs to be modified for the fracture toughness evaluation of the coating/substrate system studied in this research.

\subsection{Closing Remarks}

An overview of the basic fracture toughness models for mode I cracks was presented in this chapter. The general crack types for materials under indentation loading and the geometries of indentation impression were introduced. At macroscopic level, several types of indentation-technique-based-fracture-toughness-models for bulk materials and brittle coating/ductile substrate systems were discussed. An energy-based indentation model, which evaluates the fracture toughness of coating/substrate systems from the load-displacement curve, was reviewed. Considerable attention was paid on the stress based fracture toughness 
model developed by $\mathrm{Hu}$ and Evans [9]. The applications of this model in several researches were examined to prove its validity. The macro-level temperature-dependent fracture toughness models and their disadvantages were discussed.

At the microscopic level, various Arrhenius-type equations and the refined forms of the rate controlling equations for the strain rate and crack extension velocity estimation were particularly analyzed in this chapter. The development procedures of some significant parameters in these equations such as activation energy, activation volume and pre-exponential parameter were examined to specify the applicability and essentiality of the rate controlling theory based equations in estimating and analyzing the fracture toughness, crack growth rate and deformation behavior of materials. A well-developed micro-level temperature-dependent fracture toughness model has been used to investigate the temperature-dependent fracture toughness of various types of intermetallics and composite materials. However, some critical parameters such as dislocation density, $\rho$, plastic zone dimension, $h$, and pre-exponential factor, $J_{0}$, were assumed to be unchanged due to their inaccessibility. These problems will be further analyzed and solved in the present research, and modification of the model introduced in last section will be made to evaluate the temperature-dependent fracture toughness of $\mathrm{WC}-10 \mathrm{Co} 4 \mathrm{Cr}$ coating/1018 low carbon steel substrate system. 


\section{Chapter 3. Temperature-dependent Fracture Toughness Models Based on Crystal Structure Analysis}

To evaluate the fracture toughness of WC-10Co4Cr coating/1018 low carbon steel substrate system, an existing model based on Arrhenius-type equation is modified in this chapter.

\subsection{WC-10Co4Cr Coating/1018 Low Carbon Steel Substrate}

\section{System}

The brittle coating/ductile substrate system studied in this research consists of a composite (WC-10Co4Cr) coating and AISI 1018 low carbon steel substrate. The composite coating material is composed of $\mathrm{WC}$ and $\mathrm{CoCr}$ alloy powders. The mixed powder was sprayed onto the low carbon steel substrate via high velocity oxy fuel (HVOF) technique. The chemical composition of the composite coating material is $86 \% \mathrm{WC}, 10 \%$ Co and $4 \% \mathrm{Cr}$, corresponding to the volume ratio $76.42 \% \mathrm{WC}, 15.77 \% \mathrm{Co}$ and $7.81 \% \mathrm{Cr}$, and the stoichiometric formula of WC powder is $6.13 \mathrm{wt} \% \mathrm{C}$ and $93.87 \mathrm{wt} \% \mathrm{~W}$.

\subsection{Microstructure and Slip System of WC-10Co4Cr Composite}

To evaluate the fracture toughness of the coating/substrate system, the main phases in the composite coating and their slip systems need to be determined. 


\subsubsection{Crystal structure}

Based on the chemical composition and phase diagram shown in Figure 3-1 [82,83], the WC in the thermal sprayed $\mathrm{WC}-10 \mathrm{Co} 4 \mathrm{Cr}$ coating is present as hard $\delta$-WC phase $[84,85]$. The space group of the hexagonal crystal structure $\delta$-WC phase is $P \overline{6} m 2 P$, and the lattice parameters of $\delta$-WC phase are $a=0.2906 \mathrm{~nm}, c=0.2837 \mathrm{~nm}[82,83]$, as shown in Figure 3-2.

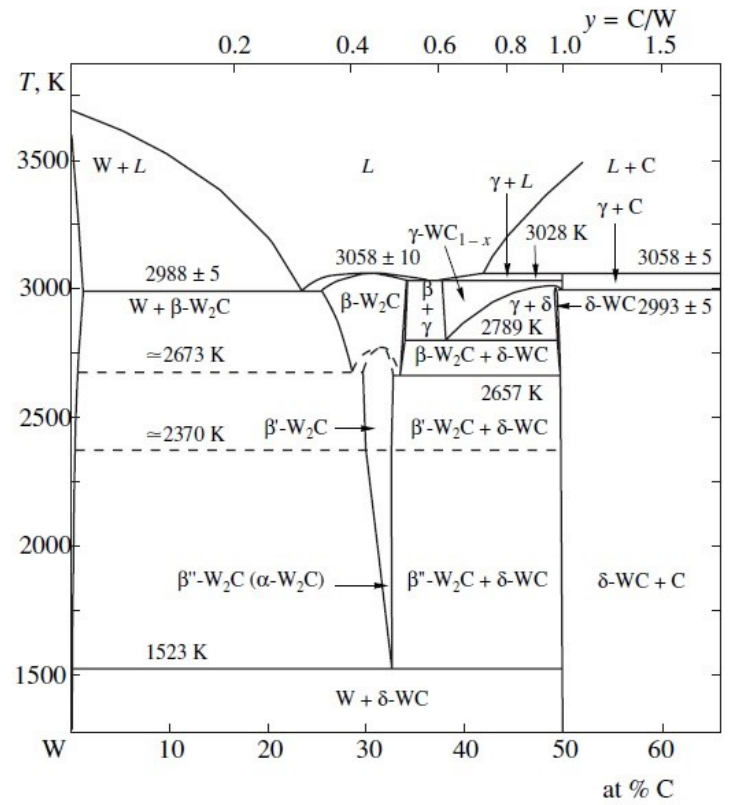

Figure 3-1. Phase diagram of tungsten carbide WC [82].
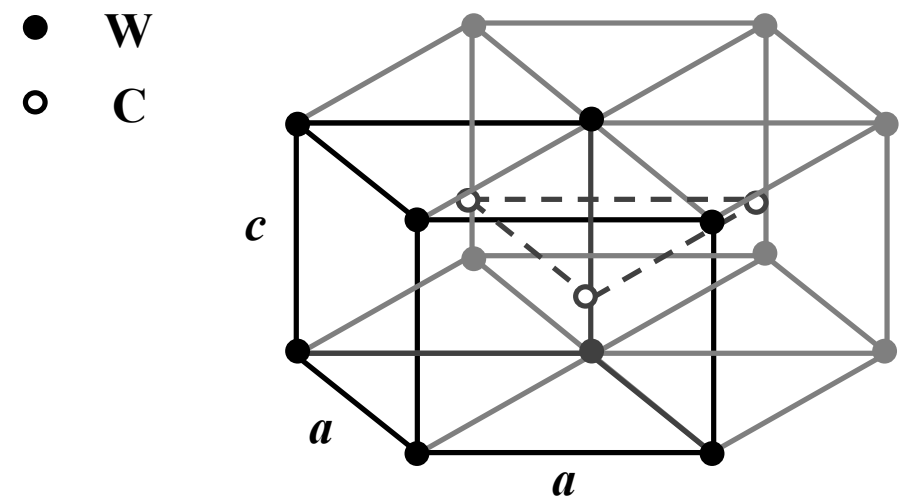

Figure 3-2. Hexagonal crystal structure of $\delta$-WC phase. 
On the other hand, the CoCr solid solution in the composite is $\varepsilon$-Co phase, which has the space group $\mathrm{P}_{3} / m m c$ of hexagonal crystal structure according to the weight fraction of $\mathrm{Cr}$ and the phase diagram shown in Figure 3-3 [86]. However, the coating thickness and processing method (HVOF) should also be considered in analyzing the microstructure of Co phase. When the powders are sprayed onto the substrate surface using the HVOF method, high spray velocity and rapid cooling rate suppress the transition of $\mathrm{CoCr}$ powder from $\alpha$-Co phase into $\varepsilon$-Co phase in the $\mathrm{WC}-10 \mathrm{Co} 4 \mathrm{Cr}$ coating. Instead, as reported from the X-ray diffraction (XRD) spectrum, in addition to the main $\delta$-WC phase, the cubic face-centered cubic (FCC) $\alpha$-Co phase was also observed in the WC-10Co4Cr coating [84-88]. In fact, the composition of WC-10Co4Cr coating is much more complex. Besides FCC $\alpha$-Co phase, $\mathrm{CO}_{3} \mathrm{~W}_{3} \mathrm{C}$ and $\mathrm{Cr}_{7} \mathrm{C}_{3}$ phases might be present in the $\mathrm{WC}-10 \mathrm{Co} 4 \mathrm{Cr}$ coating [87,88]. However, FCC $\alpha$-Co phase has also been found in other WC-Co based coatings. The CoCr solid solution in the WC-10Co4Cr coating is present as FCC $\alpha$-Co phase based on its weight fraction. Therefore, for the temperature-dependent fracture toughness calculation of WC-10Co4Cr coating/1018 low carbon steel substrate system, the $\mathrm{CoCr}$ solid solution is considered to be present as FCC $\alpha$-Co phase in the coating/substrate system, as illustrated in Figure 3-4. 


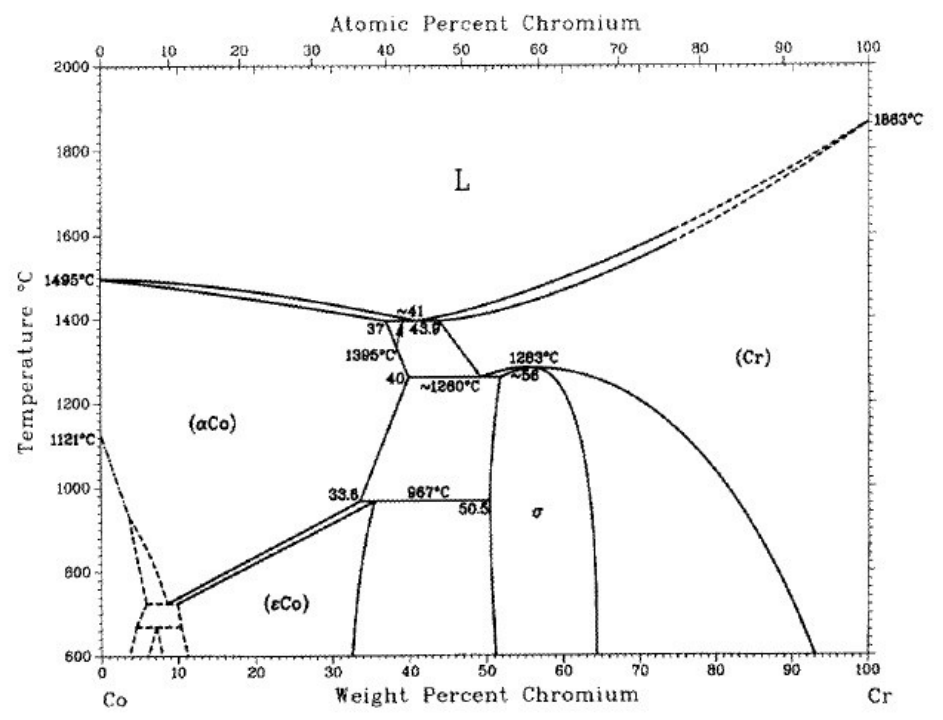

Figure 3-3. Phase diagram of cobalt-chromium $\mathrm{CoCr}[86]$.

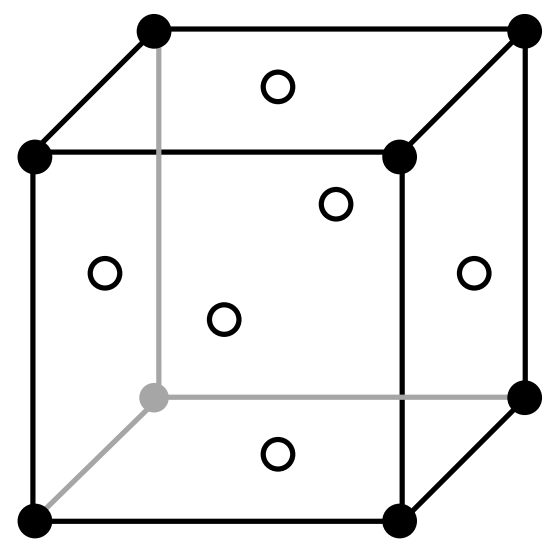

Figure 3-4. Cubic crystal structure of FCC $\alpha$-Co phase.

Due to the presence of $\mathrm{Cr}$, the lattice parameter $a$ of the ductile FCC $\alpha$-Co phase in $\mathrm{CoCr}$ solid solution is slightly larger than that in pure Co and can be calculated based on the weight percent of $\mathrm{Cr}$, as shown in Table 3-1. The body-centered cubic (BCC) crystal structure of $\mathrm{Cr}$ is not found as a single phase in the WC-10Co4Cr coating [89-90]. Based on the weight percentages of $\mathrm{Co}(71.43 \%)$ and $\mathrm{Cr}(28.57 \%)$, the space group of FCC $\alpha$-Co phase is $f m \overline{3} m$ 
and the lattice parameter can be obtained as $a=0.3561 \mathrm{~nm}$ from the fitting curve in Figure 3-5.

Table 3-1. The lattice constant $a$ of $\alpha$-Co phase at different $\mathrm{Cr}$ contents (wt $\%$ ) [86]

\begin{tabular}{|l|l|l|l|l|}
\hline & $0 \%$ & $10 \%$ & $20 \%$ & $41 \%$ \\
\hline$a(\mathrm{~nm})$ & 0.35446 & 0.3548 & 0.3554 & 0.3573 \\
\hline
\end{tabular}

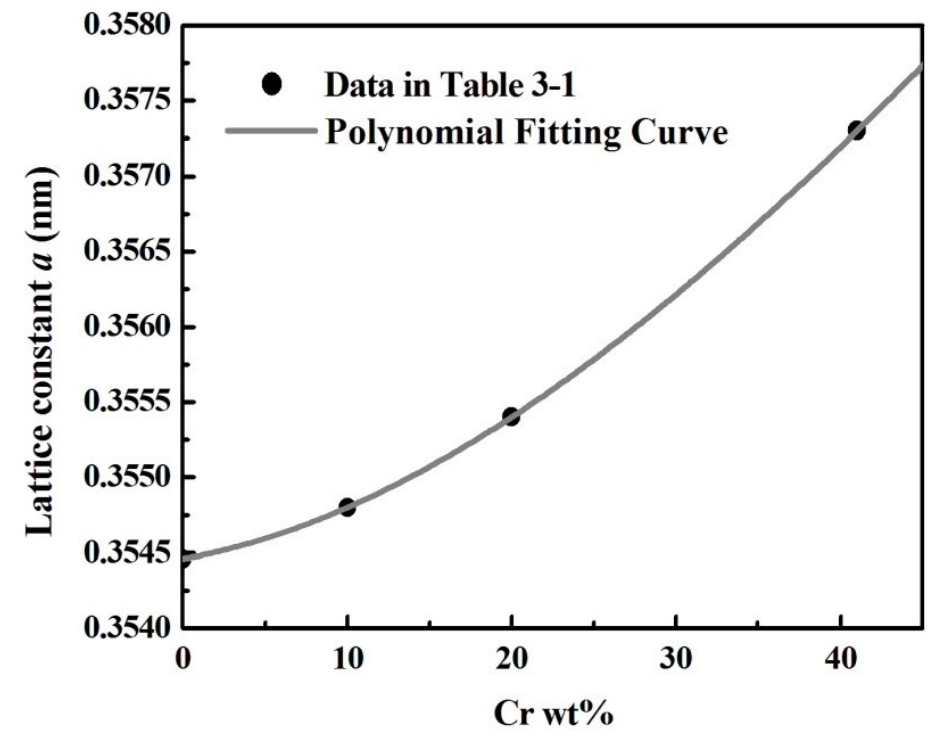

(a)

\begin{tabular}{|r|r|r|}
\hline 262 & 28.08346 & 0.35604 \\
\hline 263 & 28.20677 & 0.35605 \\
\hline 264 & 28.33008 & 0.35606 \\
\hline 265 & 28.45338 & 0.35608 \\
\hline 266 & 28.57669 & 0.35609 \\
\hline 267 & 28.7 & 0.3561 \\
\hline 268 & 28.82331 & 0.35611 \\
\hline 269 & 28.94662 & 0.35612 \\
\hline 270 & 29.06992 & 0.35613 \\
\hline
\end{tabular}

(b)

Figure 3-5. Lattice constant $a$ of FCC $\alpha$-Co phase: (a) variation with $\mathrm{Cr}$ content, (b) data from fitting curve. 


\subsubsection{Slip system}

To evaluate the fracture toughness of brittle $\delta$-WC phase and ductile $\alpha$-Co phase in the composite coating, the slip system, Burgers vector and interplanar spacing of each phase must be determined first. Since the slip system can only be observed in single-phase crystal structure, these parameters for the $\mathrm{WC}-10 \mathrm{Co} 4 \mathrm{Cr}$ composite coating are not available. However, different types of slip systems of the $\delta$-WC phase in WC-Co based composite coatings have been determined [91-93]. There are mainly three groups of Burgers vector, [1릴 $1 / 3[1 \overline{2} 13]$ and $1 / 3[1 \overline{2} 13]$ for the hard $\delta$-WC phase in the dislocation zone of WC-Co composites. Hibbs and Sinclair [94] and Marek Blanda et al. [95] reported that, when the composite materials were under indentation loading, the major slip system of the hexagonal $\delta$-WC phase in the deformed area was represented by the dislocation with the partial Burgers vector $1 / 6[1 \overline{2} 13]$ on the prismatic slip plane $\{10 \overline{1} 0\}$. Based on the observations, they deduced that the Burgers vector $1 / 3[1 \overline{2} 13]$, which consists of two same partial Burgers vectors $1 / 6[1 \overline{2} 13]$, was responsible for the primary dislocation orientation, but the partial Burgers vector of $1 / 6[1 \overline{2} 13]$ could also be a component of $1 / 3[1 \overline{2} 10]$ $(1 / 3[1 \overline{2} 10]=1 / 6[1 \overline{2} 13]+1 / 6[1 \overline{2} 1 \overline{3}])$, as shown in Figure 3-6.

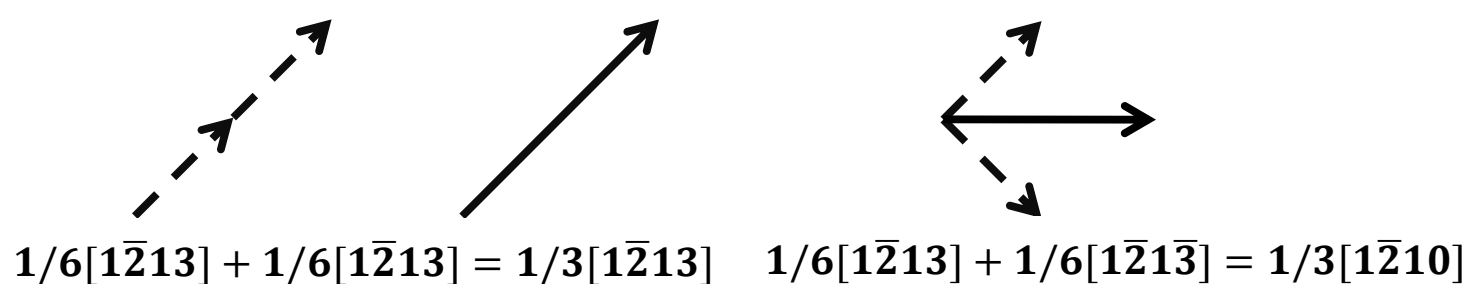

Figure 3-6. Combinations of partial Burgers vectors. 
Furthermore, in WC-Co based composite materials, only the Burgers vector of $1 / 3[1 \overline{2} 10]$ was observed at the entire surrounding area of the indentation impression where radial cracks were generated under the indentation load, whereas the Burgers vector $1 / 3[1 \overline{2} 13]$ cannot be observed in all regions of the surrounding area $[94,95]$. Therefore, for the plastic zone corresponding to the radial cracks, the Burgers vector $1 / 3[1 \overline{2} 10]$ is applied to the fracture toughness estimation of the composite coating/substrate system in this research. The magnitude of Burgers vector $1 / 3[1 \overline{2} 10]$ can then be calculated through the four-index notation, as shown in Figure 3-7. In a four-index system, the $u, v$ and $w$ axes are on the same plane and at $120^{\circ}$ apart. The vectors in dash line on each axis stand for the components of four index vector [1 $\overline{2} 10]$, respectively. The vector with the magnitude of one lattice constant and lying on negative $v$ axis is the Burgers vector $1 / 3[1 \overline{2} 10]$ on the prismatic slip plane $\{10 \overline{1} 0\}$, which corresponds to the slip system of the $\delta$-WC phase. 


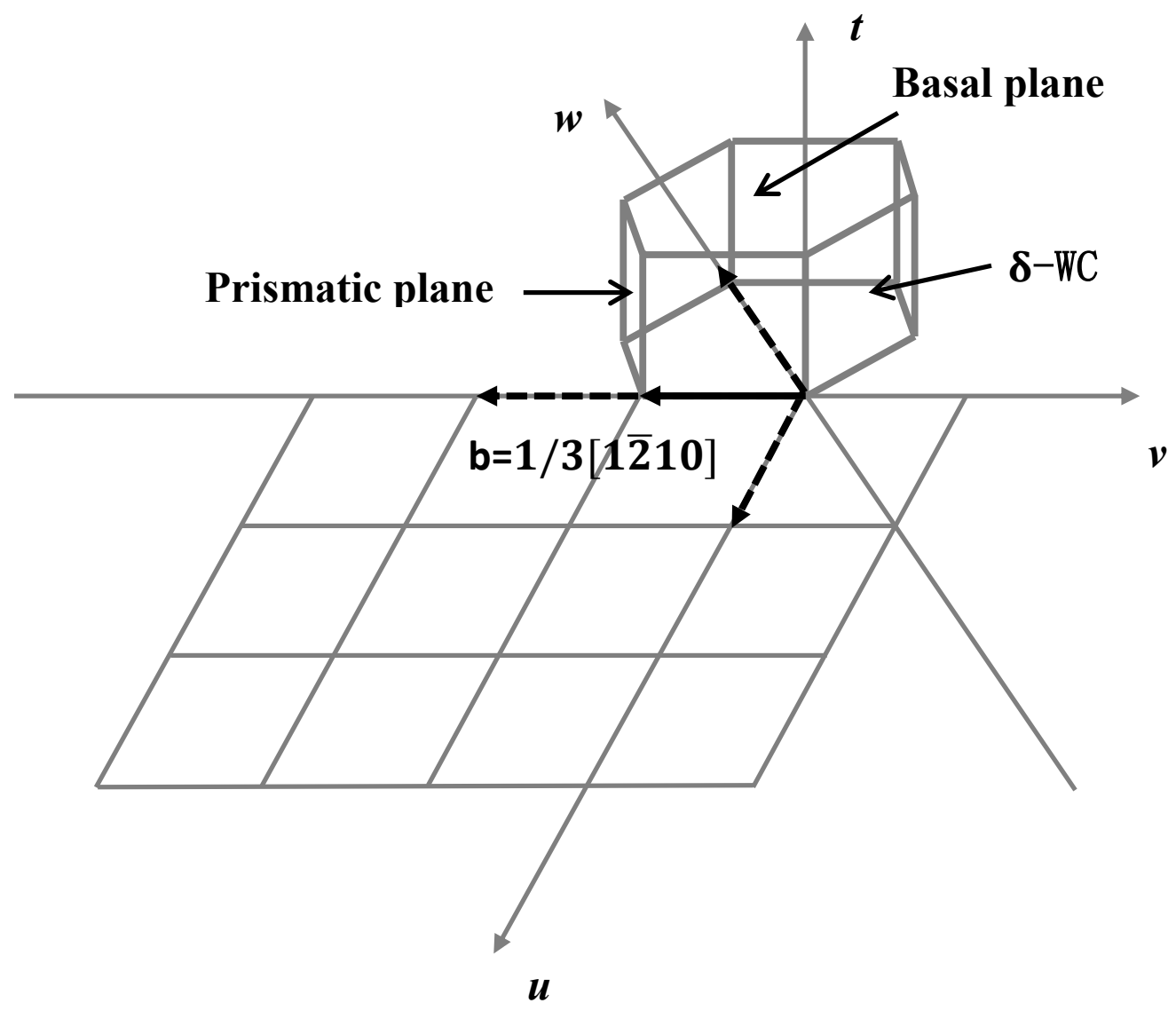

Figure 3-7. Slip system with Burgers vector $1 / 3[1 \overline{2} 10]$ in $\delta$-WC phase.

The slip system of the FCC $\alpha$-Co phase is very complex and the precise dislocation data for FCC $\alpha$-Co phase are not available [96], but the general deformation mechanism of the FCC crystal has been studied [97-99]. It has been reported that the dislocation of the FCC crystal has the Burgers vector $1 / 2[110]$ on the slip plane $\{111\}$. Furthermore, a partial Burgers vector $1 / 6[112]$ was observed by Jesser et al. [100] in the dislocation movement of FCC $\alpha$-Co phase on nickel substrate, and the Burgers vector $1 / 6[112]$ is a partial component of the Burgers vector $1 / 2[110]$. Therefore the slip system of FCC $\alpha$-Co phase can also be determined as the Burgers vector of $1 / 2[110]$ on the slip plane $\{111\}$, and the interplanar 
spacing can be calculated based on the slip system of each phase. For a $\{h k 1\}[\mathrm{uvw}]$ slip system, the magnitude of Burgers vector, $b$, can be estimated from Eq. (3-1a), and the interplanar spacing, q, can be calculated from Eq. (3-1b) for cubic FCC crystal.

$$
\begin{gathered}
|b|=\frac{a}{2} \sqrt{u^{2}+v^{2}+w^{2}} \\
q=\frac{a}{\sqrt{h^{2}+k^{2}+l^{2}}}
\end{gathered}
$$

The Burgers vector, $b$, and slip plane spacing, $q$, of both $\delta$-WC and $\alpha$-Co phases are calculated from the data discussed above and are summarized in Table 3-2.

Table 3-2. Lattice parameters of $\delta$-WC and $\alpha$-Co phases

\begin{tabular}{ccccc}
\hline & $\boldsymbol{a}(\mathbf{n m})$ & $\boldsymbol{c}(\mathbf{n m})$ & $\boldsymbol{b}(\mathbf{n m})$ & $\boldsymbol{q}(\mathbf{n m})$ \\
\hline $\boldsymbol{\delta}-\mathbf{W C}$ & 0.2906 & 0.2837 & $\mathrm{a}$ & $\sqrt{3} \mathrm{a} / 2$ \\
\hline $\boldsymbol{\alpha}-\mathbf{C o}$ & 0.3561 & - & $\mathrm{a} / \sqrt{2}$ & $\mathrm{a} / \sqrt{3}$ \\
\hline
\end{tabular}

\subsection{Model Development}

The strain energy release rate, $G$, for fracture was first defined in Irwin's work, $G \geq G_{c}=$ $2 \gamma$, the cracks grow when the strain energy release rate exceeds the critical value, $G_{c}$, which is equal to two times the surface energy. The strain energy release rate, $G$, in the material subjected to plastic deformation can be expressed as the summation of surface energy and plastic deformation energy. In Rice's work [78], the $J$ integral in crack propagation condition, $J_{c}$, is given by: 


$$
J_{c}=J_{s}+J_{d}
$$

where $J_{s}$ is elastic work which equals to $2 \gamma_{s}$ and $\gamma_{s}$ is surface energy, $J_{d}$ is plastic dissipation energy for per unit area of newly created crack.

To incorporate temperature effect into the fracture toughness calculation, the Arrhenius equation has been extensively applied to the investigation of fracture and deformation behaviors. In Arrhenius-type equation [57], the rate of thermally activated process is expressed as:

$$
\dot{\gamma}=A \exp \left(-\frac{\Delta E}{k T}\right)
$$

where $\dot{\gamma}$ is general rate or velocity of the thermally activated process, $\Delta E$ is general energy change, $A$ is a pre-exponential factor governed by the frequency of atom movement or the properties of the material, $T$ is absolute temperature and $k$ is the Boltzmann constant.

\subsubsection{Energy release based theory}

Based on the Arrhenius-type equation and $J$ integral, the temperature effect has been incorporated into the fracture toughness estimation model, which is given by [14]:

$$
\begin{gathered}
K_{I c}=\sqrt{\frac{E\left(J_{s}+J_{d}\right)}{1-v^{2}}} \\
J_{d}=J_{0} \exp \left[-\frac{b^{2}\left(\gamma_{F}+U_{P-N}\right)}{k T}\right]
\end{gathered}
$$




$$
J_{0}=\frac{\dot{\gamma}_{0} k T t}{b l \stackrel{*}{x}}
$$

where $b$ is Burgers vector, $\gamma_{F}$ is generalized stacking fault energy, $h$ is the dimension of plastic zone, $U_{P-N}$ is $(\mathrm{P}-\mathrm{N})$ barrier energy, $E$ and $v$ are Young's modulus and Poisson's ratio of the material, respectively. The density of activation lattice points, $n_{a}$, and the dislocation density, $\rho$, are introduced to calculate the temperature-dependent fracture toughness, where

$$
\begin{gathered}
n_{a}=\frac{\dot{\gamma}_{0} t}{A^{\prime}}\left(\frac{h}{l}\right) \\
\rho=\frac{n_{a}}{\pi h^{2}}
\end{gathered}
$$

Therefore, the pre-exponential term, $J_{0}$, can be described as:

$$
J_{0}=\left(\frac{k T}{b^{2}}\right) \rho \pi h^{2}
$$

The $(\mathrm{P}-\mathrm{N})$ energy barrier $U_{P-N}$ in Eq. $(3-4 \mathrm{~b})$ can be expressed by unstable stacking fault energy, $\gamma_{u s}$, and lattice phase angle, $\psi,[101]$ :

$$
\begin{gathered}
U_{P-N}=\frac{8 \pi^{2} \gamma_{u s}}{\sinh 2 \psi} \\
\gamma_{u s}=\frac{\mu_{s l i p} b^{2}}{2 \pi^{2} q} \\
\psi=\frac{\pi \kappa q}{c b}
\end{gathered}
$$

where $\mu_{\text {slip }}$ is shear modulus, $\kappa$ is dislocation line energy factor and $c$ is elastic constant. In isotropic materials, $\kappa / c$ is equal to $1 /(1-v)$ and 1 for edge dislocations and screw dislocations, respectively. The dislocation mechanisms for most composite materials are complex, which may consist of both edge and screw dislocations, and the values of $\kappa / c$ for 
different phases in the composite coating are not same due to their different microstructures and slip systems.

From Eq. (3-6), the pre-exponential term, $J_{0}$, can be interpreted as the maximum plastic work per unit area of newly created crack during crack propagation, but this expression was developed for bulk materials. Due to the mismatch of mechanical properties between the coating and substrate, the shape of plastic zone in a brittle coating/ductile substrate system is significantly different from that in a bulk material under indentation loading. Burnett et al. [102] studied the plastic zone shape in brittle coating/ductile substrate and ductile coating/brittle substrate systems through indentation tests. The relationship between the plastic zone size and the mechanical properties of the coating/substrate systems is illustrated in Figure 3-8. For hard coating/ductile substrate systems $\left(\frac{E_{c}}{H_{c}}<\frac{E_{S}}{H_{S}}\right)$, the radius of plastic zone in coating is larger than that in substrate. On the contrary, for soft coating/hard substrate system $\left(\frac{E_{c}}{H_{c}}>\frac{E_{s}}{H_{s}}\right)$, the radius of plastic zone in coating is smaller than that in substrate. 


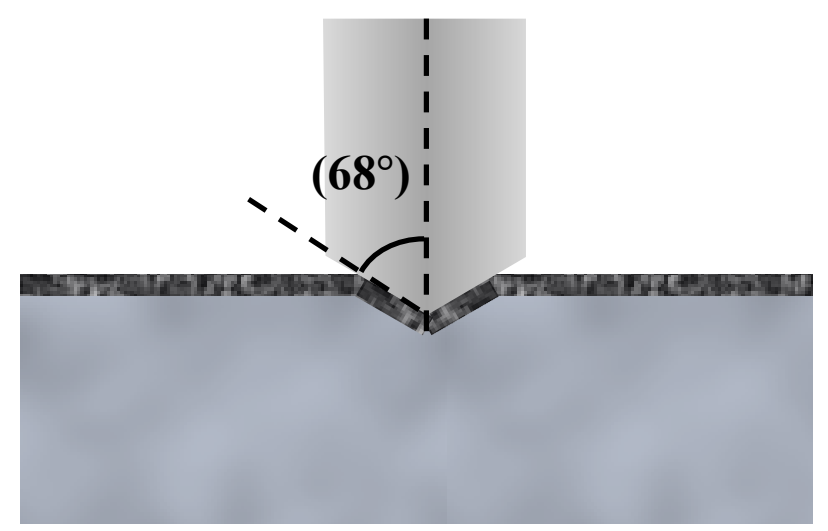

(a) Indentation test on a coating/substrate system.

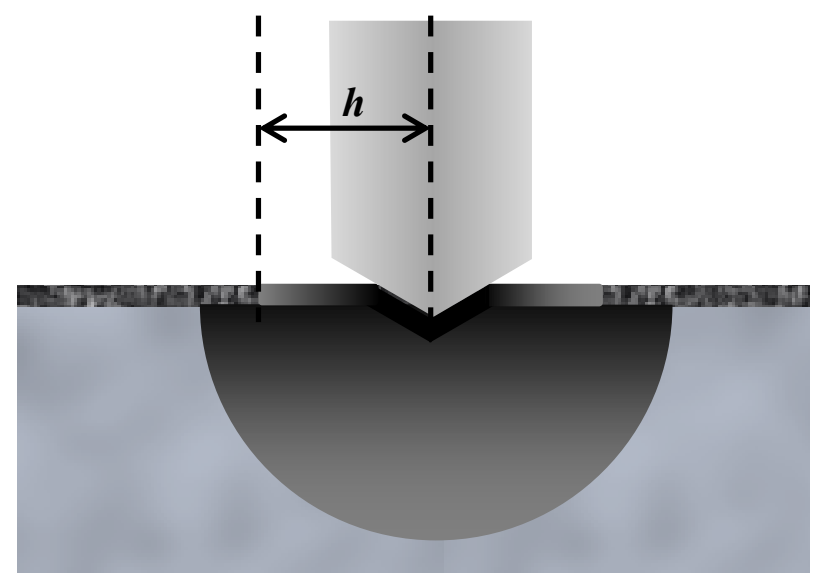

(b) $\frac{E_{c}}{H_{c}}<\frac{E_{s}}{H_{s}}$

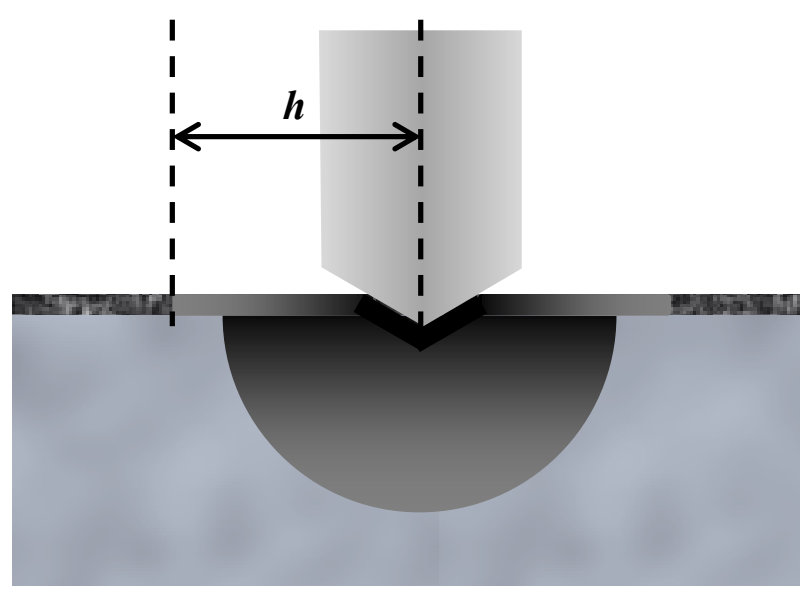

(c) $\frac{E_{c}}{H_{c}}>\frac{E_{s}}{H_{s}}$

Figure 3-8. Plastic zone in a coating/substrate system under indentation loading. 
In the indentation test-based fracture toughness model developed by Lawn et al. [15], the radial cracks initiated in the loading process would propagate at the coating surface and downward to the coating-substrate interface, and be fully developed during the unloading period $[15,16]$. The main driving force for radial crack extension is the residual stresses that are stored in the plastic zone during the loading period, while the elastic driving force is totally subordinated to the plastic driving force in this process, as observed in the WC-10Co4Cr coating/1018 low carbon substrate system. Other crack types found in the coating/substrate system under indentation loading include median crack and edge crack, which are formed and reach their full lengths during the loading process. Based on this model, the temperature-dependent fracture toughness can be evaluated through calculating the total dislocation energy stored in the plastic zone, which is responsible for the radial crack development during the unloading process. Therefore, the profiles of radial cracks developed during the unloading period are used to investigate the fracture toughness of the composite coating/substrate system.

Moreover, as shown in Figure 3-9, the fully developed radial cracks at each corner are through thickness cracks. However, there are no cracks initiated in the substrate even if the indenter penetrated into the substrate [103]. As a result, the plastic zone in the substrate has little contribution to the development of the radial cracks. 


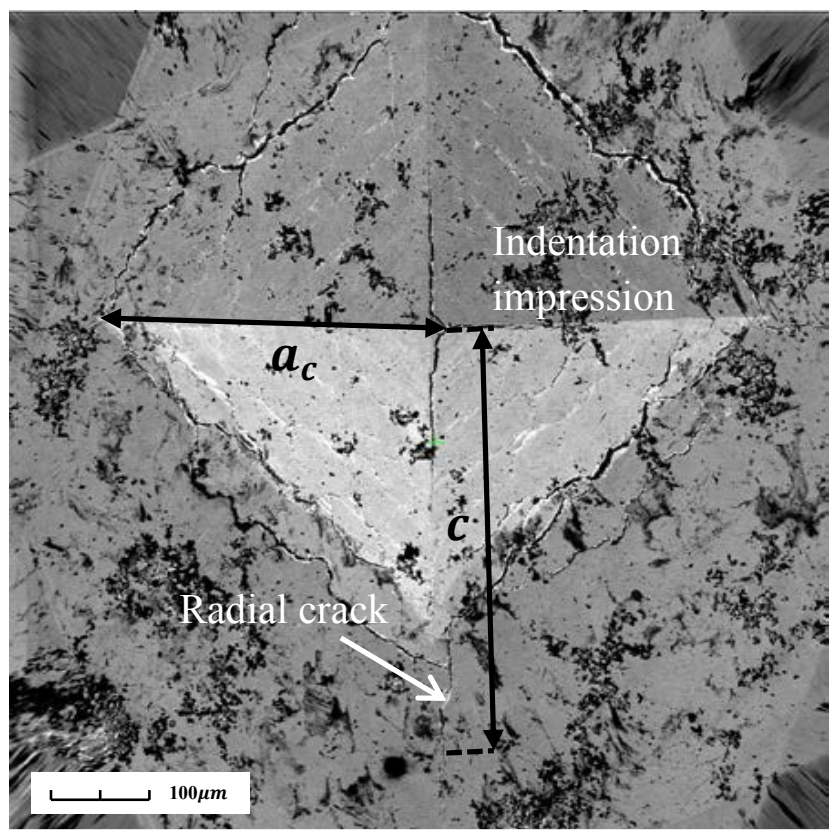

(a)

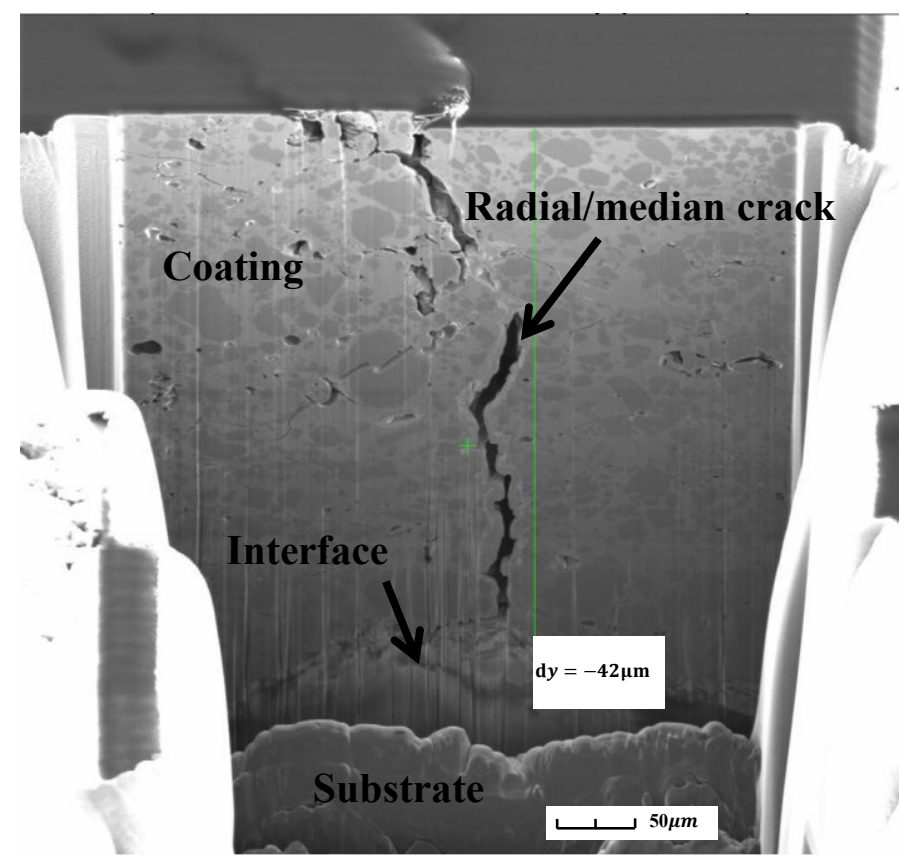

(b)

Figure 3-9. FIB images of crack profiles in WC-10Co4Cr coating/1018 steel substrate system under Vickers indentation loading: (a) top view, and (b) cross-sectional view [103]. 


\subsubsection{Crack profiles due to Vickers indentation}

Based on the model discussed above, Eq. (3-6) can be changed:

$$
J_{0}=2 \rho h t\left(\frac{k T}{b^{2}}\right)
$$

where $t$ is the coating thickness, which was measured to be $45 \mu \mathrm{m}$ [103]. For the two dimensional crack propagation problems, the pre-exponential factor, $J_{0}$, defined in Eq. (3-8) can be interpreted as the maximum plastic work per unit area of newly created radial crack in the composite coating.

The dislocation density, $\rho$, the plastic zone dimension, $h$, and the pre-exponential factor, $J_{0}$, were obtained from the density of activation lattice points, $n_{a}$. However, these critical parameters were assumed to be unchanged due to their inaccessibility. The dislocation density, $\rho$, was estimated as $3 \times 10^{11} 1 / \mathrm{m}^{2}$ for both plane strain and non-plane strain condition, the plastic zone dimension, $h$, was assumed to be $1 \mathrm{~mm}$ for plane strain condition and $2.5 \mathrm{~mm}$ for non-plane strain condition, the approximate pre-exponential parameter $J_{0}$ was $5 \times 10^{4} \mathrm{~J} / \mathrm{m}^{2}$ for plane strain condition and $3 \times 10^{5} \mathrm{~J} / \mathrm{m}^{2}$ for non-plane strain condition. Although the estimations were based on experimental data, these critical parameters should be different for each specific material and have a significant effect on the fracture toughness calculation.

The dislocation density and the size of plastic zone, however, should change with the applied load. Among diverse types of indenter geometries, the sharp edge Vickers indenter is 
commonly employed in fracture toughness tests for brittle coatings [28]. Median/radial crack system is also recognized as half-penny cracks, which may form from downward extension of the radial crack or upward propagation of the median crack or the combination of the two crack types, as shown in Figure 3-10 (a), and the median/radial crack is considered to be the major crack type in WC-10Co4Cr coating/1018 low carbon steel substrate system under an indentation load [103]. Beneath the coating surface, the plastic zone formed due to the indentation load is considered to be a semicircle-like region with a radius, $h$, as shown in Figure 3-10 (b) [15].

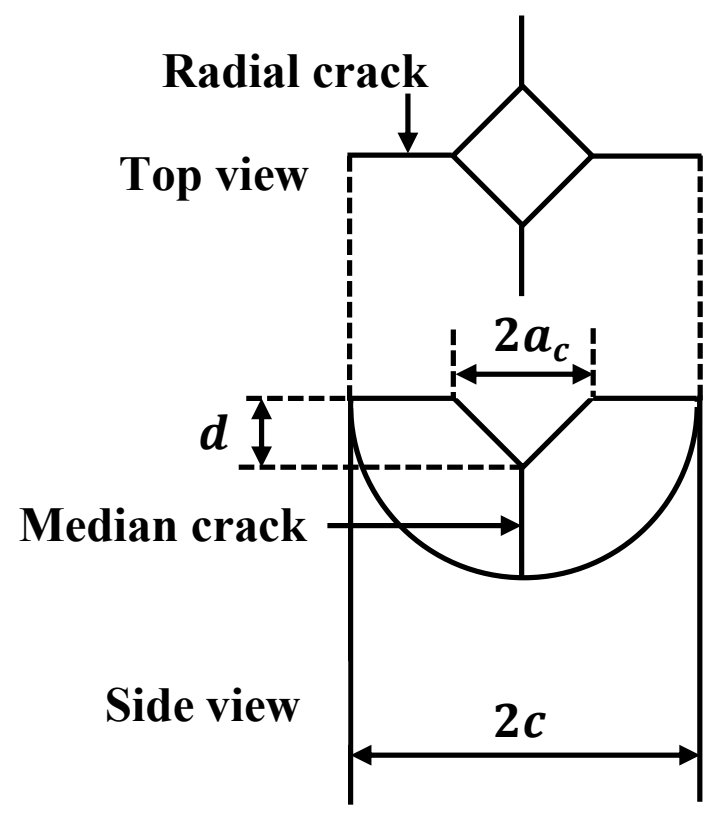

(a)

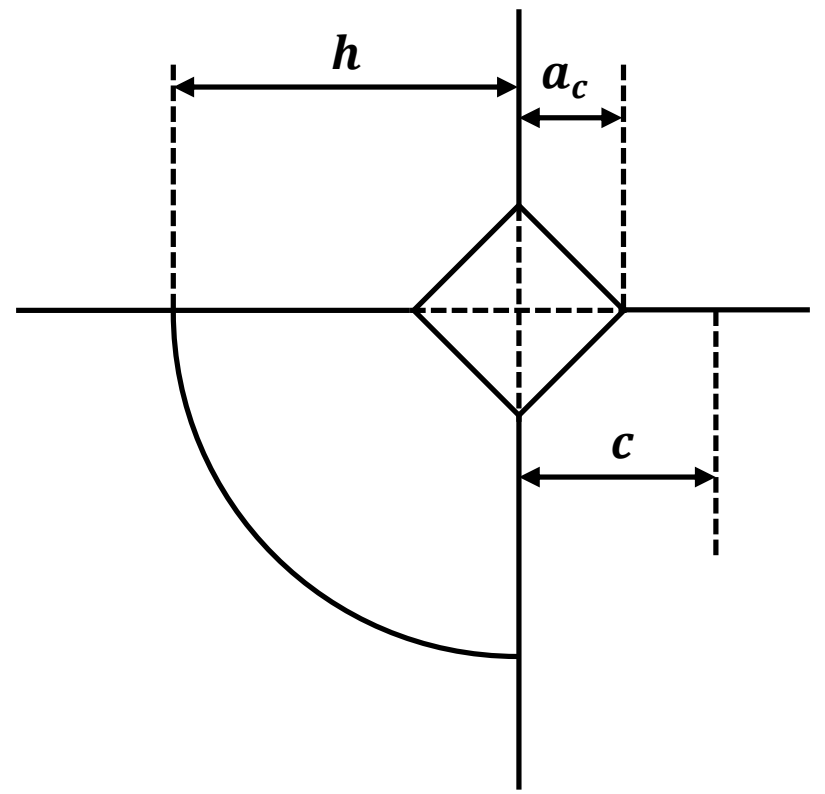

(b)

Figure 3-10. Schematic crack profiles in a solid body under Vickers indentation loading: (a) median/radial crack system, (b) indentation and plastic zone geometries. 
Based on this assumption, the general dislocation density, $\rho$, under indentation loading was described by the total length of dislocation line, $\lambda$, and indenter contact volume, $V$, in the work of Nix and Gao [104], which is given by:

$$
\rho=\frac{\lambda}{V}=\frac{\frac{\pi d a_{c}}{b}}{\frac{2}{3} \pi a_{c}^{3}}=\frac{3}{2} \frac{d a_{c}}{b a_{c}^{3}}=\frac{3}{2} \frac{\tan ^{2} \theta}{b d}
$$

where $d$ is the indentation depth, $a_{c}$ is contact radius and $\theta$ is the angle between indenter and coating surface, as shown in Figure 3-8 (a) and Figure 3-10 (b).

Durst et al. [17] took the plastic deformation into consideration and Eq. (3-9) was modified by using the volume of plastic zone, $V_{p}$, to substitute the indenter contact volume, $V$. Then the dimension of plastic zone can be related to the radius of indentation impression by applying a parameter $f$, which gives $h \approx f a_{c}$, and the dislocation density is depicted as:

$$
\rho=\frac{\lambda}{V_{p}}=\frac{\frac{\pi d a_{c}}{b}}{\frac{2}{3} \pi h^{3}}=\frac{3}{2} \frac{d a_{c}}{b f^{3} a_{c}^{3}}=\frac{3}{2} \frac{\tan ^{2} \theta}{f^{3} b d}
$$

where $f$ is a geometry related factor that can be estimated from the properties of the material and the indenter geometries. From Eq. (3-10), the expression of $\rho h$ in Eq. (3-8) can be derived as:

$$
\rho h=\frac{3}{2} \frac{d}{b f}=\frac{3}{2} \frac{\tan \theta}{b f^{2}}
$$

For isotropic plastic zone [15],

$$
f=\frac{h}{a_{c}}=\left(\frac{E_{c}}{H_{c}}\right)^{1 / 2} \cdot(\cot \psi)^{1 / 3}
$$


where $\Psi$ is the half-angle of the indenter.

Both the pre-exponential parameter $J_{0}$ in Eq. (3-4c) and the dislocation density, $\rho$, in Eq. (3-10) are load-dependent, which results in the estimated fracture toughness varying with indentation load. However, by applying the proposed model, represented by Eq. (3-8) and Eq. (3-11), the load effect can be eliminated from the final results. This assumption is reasonable since the fracture toughness is a material property and should be independent of load.

\subsubsection{Mixture models}

After the fracture toughness of $\delta$-WC and $\alpha$-Co phases are obtained based on the parameters discussed above, the fracture toughness of the WC-10Co4Cr composite coating can be determined by combining the fracture toughness of the two phases. Two mixture models for the fracture toughness evaluation of two-phase composite materials are applied in this research. The first one is derived from the basic mixture rule of $J$-integral, $J_{c}=V_{f} J_{b}+(1-$ $\left.V_{f}\right) J_{d}$, where $J_{c}$ is the critical $J$-integral for crack propagation, $J_{b}$ and $J_{d}$ are brittle and ductile components, respectively. The fracture toughness, $K_{c}$, of a composite material that contains a brittle phase with fracture toughness, $K_{b}$, and a ductile phase with fracture toughness, $K_{d}$, is given by [105]:

$$
K_{c}=K_{b}\left[V_{f}+\left(1-V_{f}\right)\left(\frac{K_{d}}{K_{b}}\right)^{2}\right]^{1 / 2}
$$

where $V_{f}$ is the volume fraction of the brittle phase. 
Bower and Ortiz [106] indicated that trapping, bowing, and bridging phenomena might appear at crack tip area and affect the fracture resistance of a composite material to some extent. Therefore, they developed the unconstrained mixture model for estimating the fracture toughness of two-phase composites. Thus the fracture toughness, $K_{c}$, is given as:

$$
\begin{gathered}
K_{c}=K_{b}\left[1+Z \sqrt{1-V_{f}}\left(\left(\frac{K_{d}}{K_{b}}\right)^{2}-1\right)\right]^{1 / 2} \\
Z=\frac{2}{\sqrt{\pi}}
\end{gathered}
$$

The applicability of this unconstrained mixture model has been verified through estimating the fracture toughness of a two-phase WC-Co composite. By comparing the calculated results with the existing experimental data [105-107], the unconstrained mixture model showed good suitability for the WC-Co composite when the volume fraction of cobalt was less than $40 \%$. The fracture toughness of the $\mathrm{WC}-10 \mathrm{Co} 4 \mathrm{Cr}$ composite coating is evaluated using these two models and the results are compared in the present research.

\subsection{Results of Fracture Toughness}

The mechanical properties of the WC-10Co4Cr coating and 1018 low carbon steel substrate materials are given in Table 3-3. The half-angle of the indenter is $1.1868 \mathrm{rad}\left(68^{\circ}\right)$, as shown in Figure 3-8(a), and the value of the parameter $f$ in Eq. (3-12) is 4.1162 for the composite coating/substrate system based on the data in Table 3-3. 
Table 3-3. Mechanical properties of WC-10Co4Cr coating and 1018 low carbon steel substrate materials [18]

\begin{tabular}{cccc}
\hline Material & $\boldsymbol{E}(\mathrm{GPa})$ & $\boldsymbol{H}(\mathrm{GPa})$ & $\boldsymbol{v}$ \\
\hline WC-10Co4Cr & 310 & 10.4 & 0.3 \\
\hline $\mathbf{1 0 1 8}$ low carbon steel & 250 & 1.05 & - \\
\hline
\end{tabular}

\subsubsection{Determination of model parameters}

For further calculations, the generalized stacking fault energy, $\gamma_{F}$, the surface energy, $\gamma_{s}$, shear modulus, $\mu_{\text {slip }}$, Young's modulus and Poisson's ratio are required. These parameters are given in Table 3-4. The generalized stacking fault energy, $\gamma_{F}$, is $0.05 \mathrm{~J} / \mathrm{m}^{2}$ for $\delta$-WC phase [108] and $0.0135 \mathrm{~J} / \mathrm{m}^{2}$ for $\alpha$-Co phase [109]. The surface energies, $\gamma_{s}$, of $\delta$-WC phase and $\alpha$-Co phase are $2.64 \mathrm{~J} / \mathrm{m}^{2}[110]$ and $2.7 \mathrm{~J} / \mathrm{m}^{2}[111,112]$, respectively. However, the surface energy, $\gamma_{s}$, for $\delta$-WC phase given in literature varies from $2 \mathrm{~J} / \mathrm{m}^{2}$ to $3.7 \mathrm{~J} / \mathrm{m}^{2}$ [110,113,114].

Table 3-4. Required parameters for the temperature-dependent fracture toughness calculation

\begin{tabular}{ccccc}
\hline & $\gamma_{s}\left(\mathrm{~J} / \mathbf{m}^{2}\right)$ & $\mu_{\text {slip }}(\mathbf{G P a})$ & $\boldsymbol{E}(\mathbf{G P a})$ & $\boldsymbol{v}$ \\
\hline $\boldsymbol{\delta}$-WC & 2.64 & 269 & 668.35 & 0.24 \\
\hline $\boldsymbol{\alpha}-\mathrm{Co}$ & 2.7 & 75 & 209 & 0.31 \\
\hline
\end{tabular}


The last parameter needs to be determined is $\kappa / c$ in Eq. $(3-7 \mathrm{c})$, which is related to the dislocation type of each phase. In the $\delta$-WC phase, the selected Burgers vector $1 / 3[1 \overline{2} 10]$ is observed on the prismatic slip plane [115]. On the prismatic slip plane, the dislocation type contains both edge dislocation and screw dislocation, and the ratio of edge dislocation to screw dislocation is three to one based on the slip angle on this specific plane for WC $[116,117]$

\subsubsection{Fracture toughness of $\delta$-WC phase}

Using the parameters discussed above, the $\mathrm{P}-\mathrm{N}$ barrier energy, $U_{P-N}$, the unstable stacking fault energy, $\gamma_{u s}$, and lattice phase angle, $\psi$, for $\delta$-WC phase can be calculated and the results are reported in Table 3-5.

Table 3-5. Calculation results of crystal structure parameters for $\delta$-WC phase

\begin{tabular}{cccc}
\hline & $\boldsymbol{U}_{\boldsymbol{P}-\boldsymbol{N}}\left(\mathrm{J} / \mathbf{m}^{2}\right)$ & $\boldsymbol{\gamma}_{\boldsymbol{u s}}\left(\mathrm{J} / \mathbf{m}^{2}\right)$ & $\boldsymbol{\psi}(\mathbf{r a d})$ \\
\hline $\boldsymbol{\delta}$-WC & 0.8613 & 4.5723 & 3.3657 \\
\hline
\end{tabular}

The temperature-dependent fracture toughness of single-phase $\delta$-WC coating is calculated with the values of $U_{P-N}, \gamma_{u s}$ and $\psi$ over a temperature range from $1 \mathrm{~K}$ to $1000 \mathrm{~K}$ using MATLAB 2010b, and the results are illustrated in Figure 3-11. 


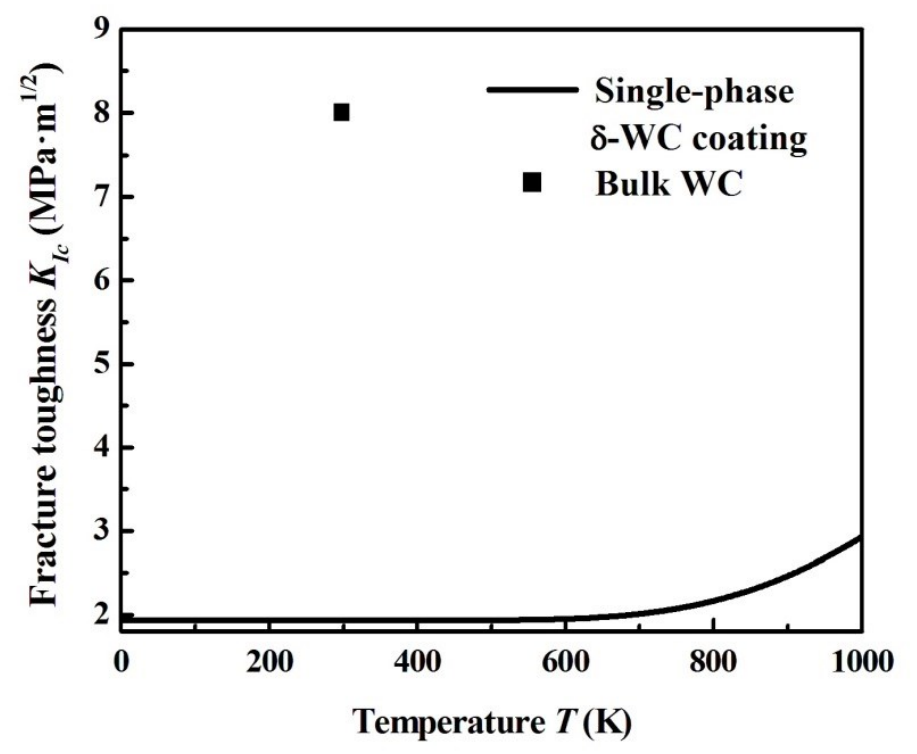

Figure 3-11. The calculated temperature-dependent fracture toughness versus temperature for single-phase $\delta$-WC coating and a measured room-temperature fracture toughness value of bulk WC [118].

It is seen that the fracture toughness of single-phase $\delta$-WC coating at room temperature $(298 \mathrm{~K})$ is $1.9346 \mathrm{MPa} \cdot \mathrm{m}^{1 / 2}$. It has been reported that the fracture toughness of bulk WC-Co alloy is $8 \mathrm{MPa} \cdot \mathrm{m}^{1 / 2}$ at room temperature when the Co weight fraction is zero [118], and this value is much greater than the calculated result for $\delta$-WC coating in this research. It is understandable, because coating thickness is one of the decisive parameters for the fracture toughness estimation [9]. For the HVOF WC-10Co4Cr coating at room temperature, Cantera and Mellor [119] measured the fracture toughness using indentation technique, and reported that based on Evans and Wilshaw equations the fracture toughness parallel to the coating/substrate interface was $5.1 \mathrm{MPa} \cdot \mathrm{m}^{1 / 2}$ and $6.1 \mathrm{MPa} \cdot \mathrm{m}^{1 / 2}$ for $\mathrm{D}$-gun deposition method and HVOF processing method, respectively. According to Lawn Fuller equations, the 
fracture toughness was $4.7 \mathrm{MPa} \cdot \mathrm{m}^{1 / 2}$ for D-gun deposition method and $5.8 \mathrm{MPa} \cdot \mathrm{m}^{1 / 2}$ for HVOF processing method. Wood [120] reported that the fracture toughness of WC-10Co4Cr coating was within the range from $2.78 \mathrm{MPa} \cdot \mathrm{m}^{1 / 2}$ to $3.85 \mathrm{MPa} \cdot \mathrm{m}^{1 / 2}$ due to the different coating thicknesses, carbide sizes and coating processing methods. Chivavibul et al. [121] investigated the influence of different processing methods on the fracture toughness of WC-Co coating, and reported that the fracture toughness of HVOF WC-Co coating with 15 $\mathrm{wt} \%$ Co was in the range from $3 \mathrm{MPa} \cdot \mathrm{m}^{1 / 2}$ to $4 \mathrm{MPa} \cdot \mathrm{m}^{1 / 2}$ for different grain sizes, and this result was much smaller than that of bulk WC-Co alloy. Demidova et al. [103] obtained the fracture toughness of WC-10Co4Cr coating/1018 low carbon steel substrate system which had an average value $K_{I C}=1.21 \mathrm{MPa} \cdot \mathrm{m}^{1 / 2}$. Therefore, for the brittle $\delta$-WC coating, the fracture toughness calculated from the present work is reasonable, since it is apparently smaller than that of bulk WC-Co alloy.

The calculated fracture toughness is also plotted against temperature in Figure 3-11. The fracture toughness of brittle $\delta$-WC coating has an increment from $1.9346 \mathrm{MPa} \cdot \mathrm{m}^{1 / 2}$ to 2.9306 $\mathrm{MPa} \cdot \mathrm{m}^{1 / 2}$ at elevated temperatures. The estimated fracture toughness of $\delta$-WC coating remains unchanged until the temperature reaches about $600 \mathrm{~K}$. This behavior is caused by the temperature-independent elastic component, $J_{s}$. At relative low temperatures (below $600 \mathrm{~K}$ for $\delta$-WC), as demonstrated by Eq. (3-4a), the work done by plastic deformation can be ignored, while the work done by elastic deformation in terms of the surface energy, $2 \gamma_{s}$, dominates the strain energy release rate of the hard $\delta$-WC coating 
during the crack propagation process. When the temperature reaches a critical value $(600 \mathrm{~K})$, the value of the plastic component, $J_{d}$, increases rapidly, and the work done by plastic deformation exceeds that done by elastic deformation and dominates the strain energy release rate when it reaches about $950 \mathrm{~K}$, as shown in Figure 3-12. Therefore, the fracture toughness of $\delta$-WC coating has a rapid increase above $600 \mathrm{~K}$.

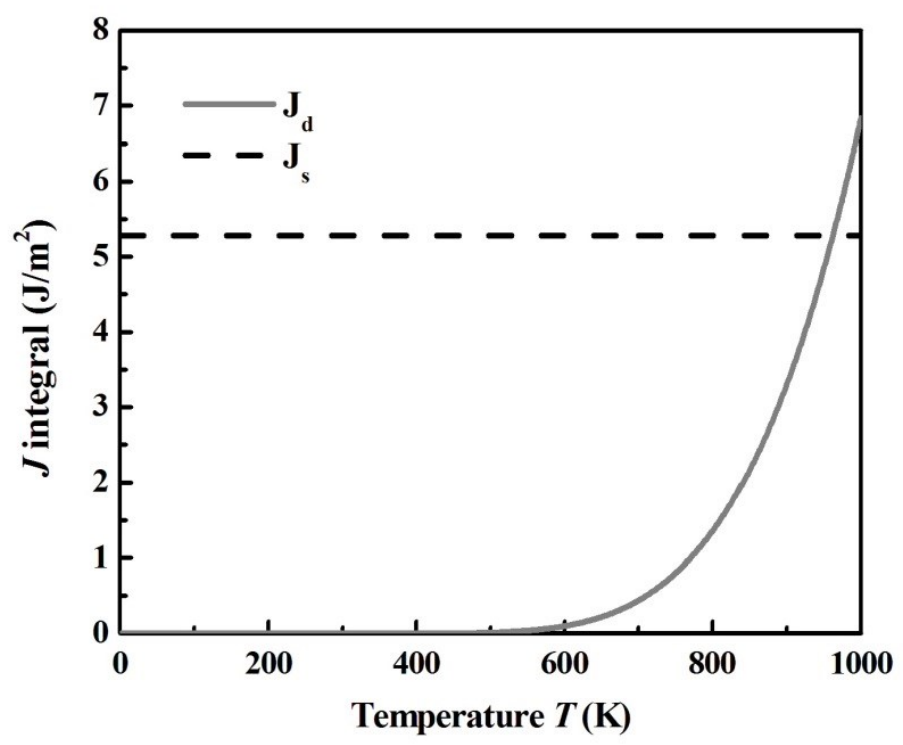

Figure 3-12. Comparison between elastic work $J_{s}$ and plastic dissipation energy $J_{d}$ for single-phase $\delta$-WC coating over a temperature range from $1 \mathrm{~K}$ to $1000 \mathrm{~K}$.

The temperature-dependent fracture toughness of single-phase $\delta$-WC coating is acceptable when compared with the existing data. It has been reported that the fracture toughness of several types of WC-Co based alloys are independent of temperature until it reaches about $600{ }^{\circ} \mathrm{C}$, as shown in Fig. 3-13 [122]. However, these results were obtained with certain approximation, and reliable experimental data are deficient. 


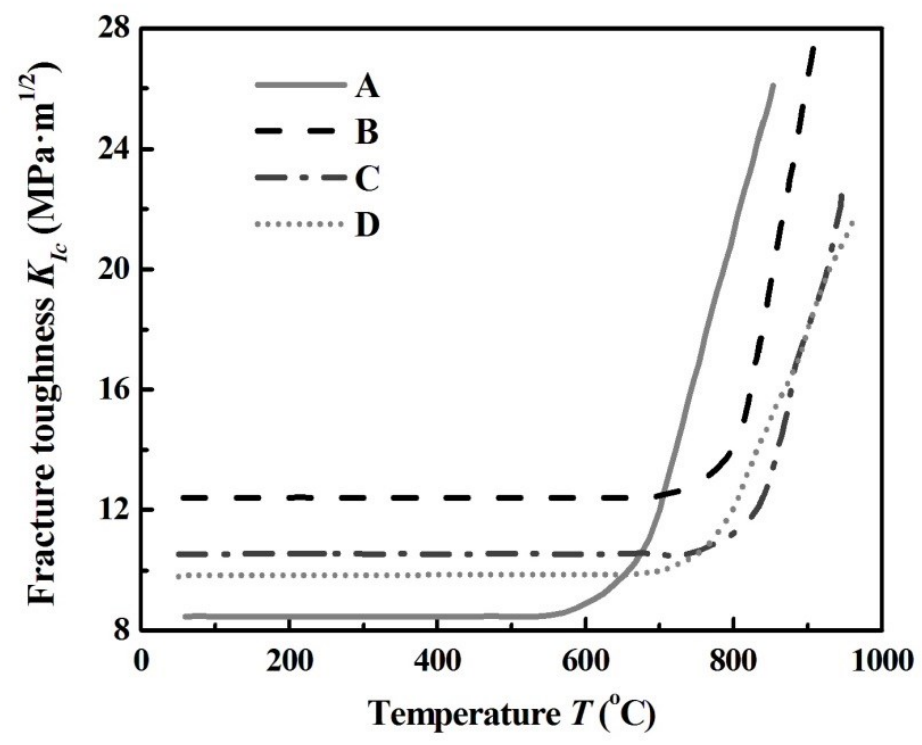

Figure 3-13. Fracture toughness of WC-Co based alloys: A for $86 \mathrm{WC} 2 \mathrm{TaC} 12 \mathrm{Co}$; B for 85WC9(Ti,Ta,Nb)C6Co; C for 80WC12(Ti,Ta,Nb)C8Co; D for 96WC-4Co [122].

\subsubsection{Fracture toughness of $\alpha$-Co phase}

For the cubic FCC $\alpha$-Co phase, the dislocation type could not be determined easily since the precise dislocation data for FCC $\alpha$-Co phase in $\mathrm{WC}-10 \mathrm{Co} 4 \mathrm{Cr}$ composite are not available [96]. Similar to the dislocation in $\delta$-WC phase, it consists of both screw dislocation and edge dislocation under indentation loading. The Burgers vector of $1 / 2[110]$ on the slip plane $\{111\}$ could point to either edge dislocation or screw dislocation [97,123], and one of its partial dislocations, the Burgers vector of $1 / 6[112]$, which is corresponding to the edge dislocation, has been observed in the thin Co film deposited on nickel substrate [100]. Since the dislocation type for $\alpha$-Co phase is very complex, the value of $\kappa / c$ in Eq. (3-7c) for $\alpha$-Co phase ranges from 1 to 1.4493 based on the equations presented previously, and should be determined via further analysis. The calculated fracture toughness of single-phase $\alpha$-Co 
coating is plotted against the ratio of $\kappa / c$ ranging from 1 to 1.445 at room temperature (298 K), as shown in Figure 3-14.

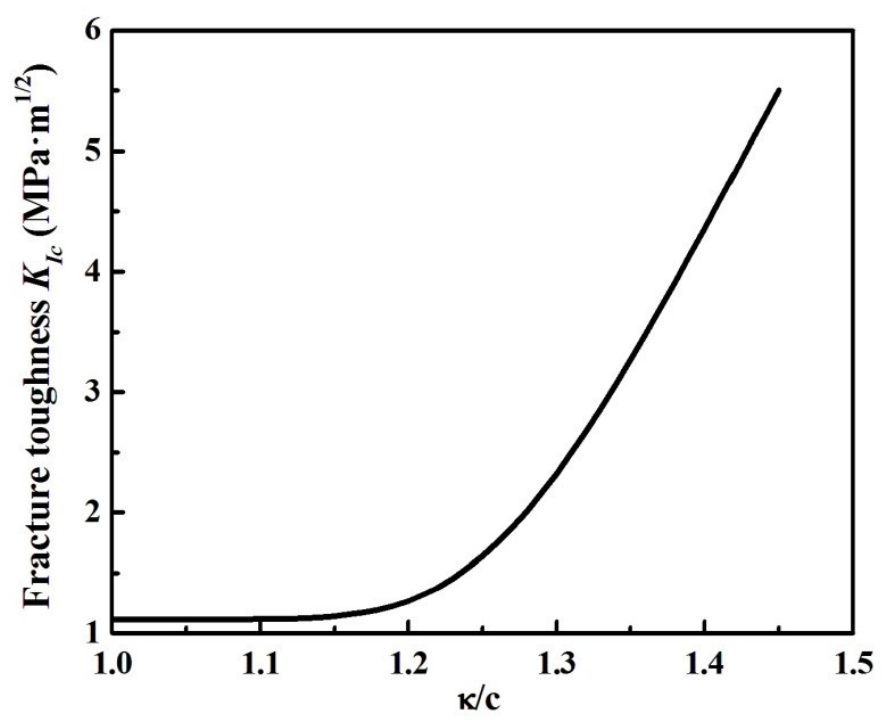

Figure 3-14. Fracture toughness variation of single-phase $\alpha$-Co coating with the ratio of $\kappa / c$.

It is shown that the room temperature fracture toughness of single-phase $\alpha$-Co coating increases with the $\kappa / c$ ratio significantly when the ratio is greater than about 1.2 . The value of $\kappa / c$ is finally selected as 1.3 based on two considerations. First, both the edge dislocation and screw dislocation are observed in FCC $\alpha$-Co phase. Second, the fracture toughness of the relative ductile Co coating should be greater than that of hard WC coating. The fracture toughness of $\delta$-WC coating at room temperature is $1.9346 \mathrm{MPa} \cdot \mathrm{m}^{1 / 2}$, thus the parameter $\kappa / c$ should be equal or greater than 1.28 according to Figure 3-14 (the calculated fracture toughness of $\alpha$-Co phase is $1.8778 \mathrm{MPa} \cdot \mathrm{m}^{1 / 2}$ for $\mathrm{\kappa} / c=1.27$ and $2.0153 \mathrm{MPa} \cdot \mathrm{m}^{1 / 2}$ for $\kappa / c=1.28)$. Although the ratio of two dislocation types for the FCC $\alpha$-Co phase is not 
reported, the parameter $\kappa / c=1.3$ can reflect the combination of the two dislocation types more properly than the larger values such as 1.4 , since the larger values indicate that edge dislocation dominates the dislocation type in $\alpha$-Co phase. By assuming the parameter $\kappa / c$ is 1.3 , the calculated fracture toughness of the $\mathrm{WC}-10 \mathrm{Co} 4 \mathrm{Cr}$ composite coating is comparable with the results obtained by other researchers. The calculated $\mathrm{P}-\mathrm{N}$ barrier energy, $U_{P-N}$, the unstable stacking fault energy, $\gamma_{u s}$, and lattice phase angle, $\psi$, for FCC $\alpha$-Co phase, with $\kappa / c=1.3$, are reported in Table 3-6.

Table 3-6. Calculation results of crystal structure parameters for $\alpha$-Co phase

\begin{tabular}{cccc}
\hline & $\boldsymbol{U}_{\boldsymbol{P}-\boldsymbol{N}}\left(\mathrm{J} / \mathbf{m}^{2}\right)$ & $\gamma_{u s}\left(\mathrm{~J} / \mathbf{m}^{2}\right)$ & $\boldsymbol{\psi}(\mathbf{r a d})$ \\
\hline $\boldsymbol{\alpha - C o}$ & 0.2348 & 1.1717 & 3.3347 \\
\hline
\end{tabular}

The fracture toughness of single-phase $\alpha$-Co phase is calculated using the data in Table 3-6 over a range of temperature from $1 \mathrm{~K}$ to $1000 \mathrm{~K}$, as presented in Figure 3-15. It is seen that the calculated fracture toughness of FCC $\alpha-\mathrm{Co}$ phase is $2.3256 \mathrm{MPa} \cdot \mathrm{m}^{1 / 2}$ at room temperature. The fracture properties of pure Co or CoCr alloy have rarely been studied [96], but Marrey et al. [124] reported that the approximate fracture toughness of CoCr alloy was $60 \mathrm{MPa} \cdot \mathrm{m}^{1 / 2}$. Similar to the situation for $\delta$-WC phase, the fracture toughness of $\alpha$-Co phase in a coating/substrate system should be much lower than that in bulk materials. 


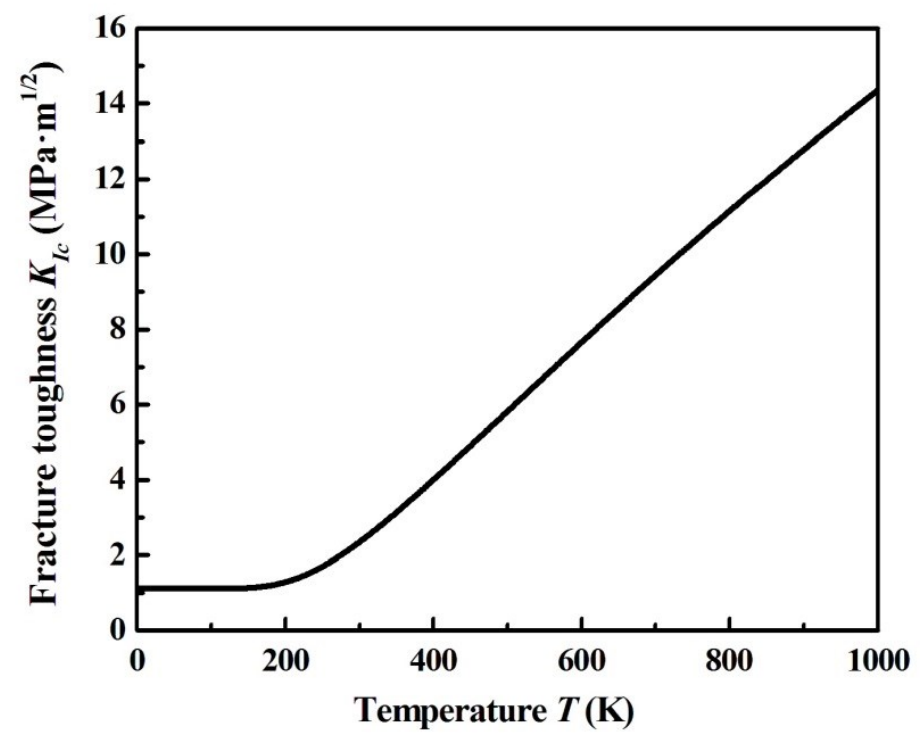

Figure 3-15. Temperature-dependent fracture toughness of single-phase $\alpha$-Co coating.

The temperature-dependent fracture toughness of single-phase $\alpha$-Co coating is quite different from that of $\delta$-WC coating, comparing the curve in Figure 3-11 with that in Figure 3-15. The fracture toughness of $\alpha$-Co coating has a significant increase from $2.3256 \mathrm{MPa} \cdot \mathrm{m}^{1 / 2}$ to 14.366 $\mathrm{MPa} \cdot \mathrm{m}^{1 / 2}$ when the temperature increases from $298 \mathrm{~K}$ to $1000 \mathrm{~K}$. It begins to increase when the temperature reaches about $200 \mathrm{~K}$. However, this phenomenon is not observed on single-phase $\delta$-WC coating. The melting point of element Co is $1768 \mathrm{~K}$, and the melting point of $\mathrm{WC}$ is $3143 \mathrm{~K}$. Apparently, even though the melting point of element $\mathrm{Cr}$ is $2179 \mathrm{~K}$, in the Co-dominant $\alpha$-Co coating (weight fraction of $\mathrm{Co}$ is $71.43 \%$ in the CoCr solid solution), the temperature effect on the mechanical properties should be much larger than that in $\delta$-WC coating. This may explain the difference in calculated fracture toughness between the two single-phase coatings at elevated temperatures. As demonstrated in Figure 3-16, the 
apparent growth of the work done by plastic deformation $J_{d}$ starts at about $200 \mathrm{~K}$, and begins to dominate the strain energy release rate from about $250 \mathrm{~K}$, which means that the $\alpha$-Co phase is very sensitive to the temperature variation even at relative low temperatures.

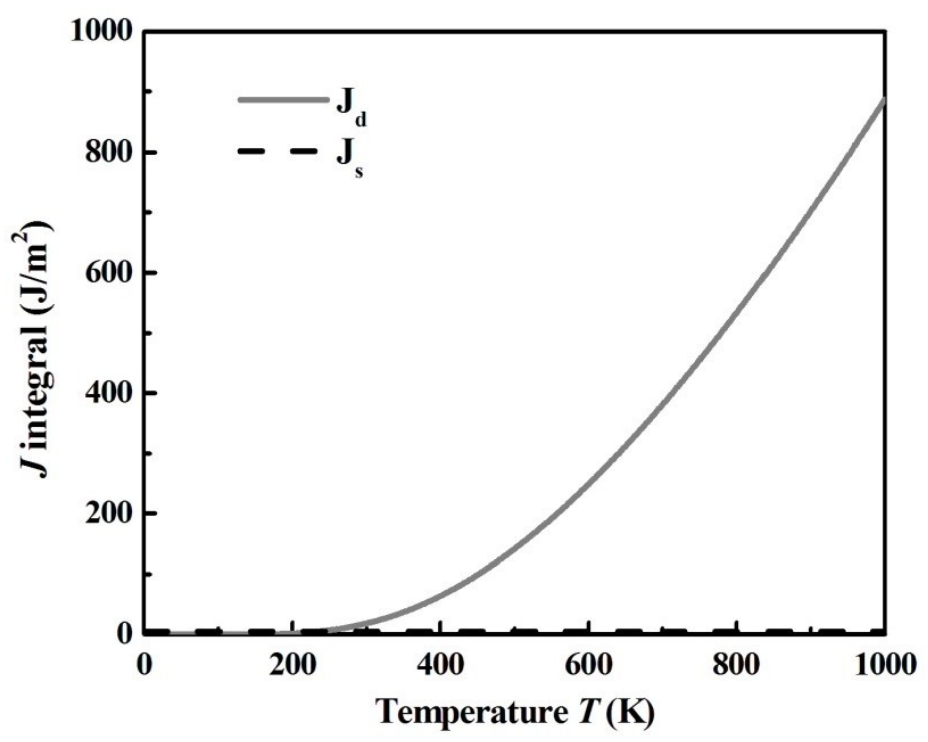

Figure 3-16. Comparison between elastic work $J_{s}$ and plastic dissipation energy $J_{d}$ for single-phase $\alpha$-Co coating over a temperature range from $1 \mathrm{~K}$ to $1000 \mathrm{~K}$.

\subsubsection{Fracture toughness of $\mathrm{WC}-10 \mathrm{Co} 4 \mathrm{Cr}$ composite}

Based on the calculated fracture toughness values of $\delta$-WC and $\alpha$-Co coatings, the fracture toughness of WC-10Co4Cr coating/1018 low carbon steel substrate system can be obtained from the unconstrained mixture method according to Eq. (3-14), as plotted in Figure 3-17. The room-temperature fracture toughness of the composite coating is $2.1576 \mathrm{MPa} \cdot \mathrm{m}^{1 / 2}$. The room-temperature fracture toughness values of other similar coatings are also given in Figure 3-17 for comparison. 


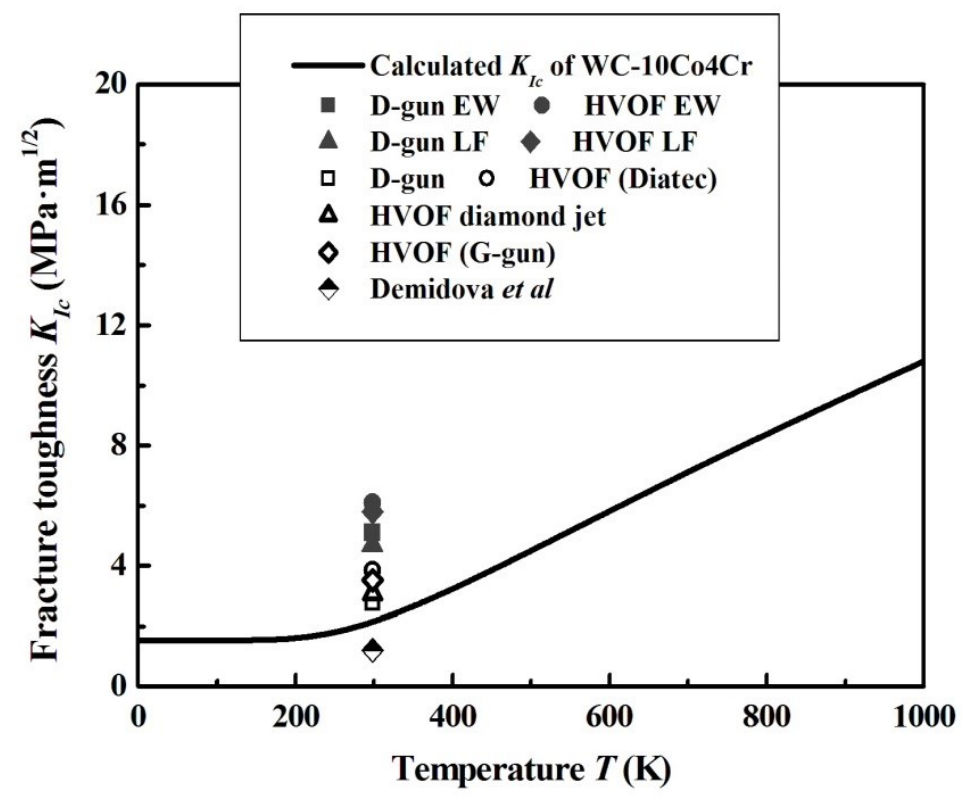

Figure 3-17. Temperature-dependent fracture toughness of WC-10Co4Cr coating/1018 low carbon steel substrate system over a temperature range from $1 \mathrm{~K}$ to $1000 \mathrm{~K}$ with the experimental data of other similar coatings for comparison $[18,119,120]$.

For the coating/substrate systems with exactly same chemical composition of coating material, the same substrate and same deposition method, the fracture toughness of these coating/substrate systems may not be same due to their different coating thicknesses, grain sizes and other critical parameters. Therefore, as discussed previously, the estimated room-temperature fracture toughness, $K_{I C}=2.1576 \mathrm{MPa} \cdot \mathrm{m}^{1 / 2}$, is in a reasonable range for WC-10Co4Cr coating/1018 low carbon steel substrate system when compared with the experimental data given in literature. Cantera and Mellor [119] reported that for WC-10Co4Cr composite coating, the fracture toughness parallel to the coating/substrate interface was $5.1 \mathrm{MPa} \cdot \mathrm{m}^{1 / 2}$ for D-gun coating processing method and $6.1 \mathrm{MPa} \cdot \mathrm{m}^{1 / 2}$ for HVOF coating process, based on the Evans and Wilshaw equations. Using the Lawn and 
Fuller equations, the fracture toughness was $4.7 \mathrm{MPa} \cdot \mathrm{m}^{1 / 2}$ for D-gun coating processing method and $5.8 \mathrm{MPa} \cdot \mathrm{m}^{1 / 2}$ for HVOF processing method. Wood [120] obtained that the fracture toughness of $\mathrm{WC}-10 \mathrm{Co} 4 \mathrm{Cr}$ coating varied from $2.78 \mathrm{MPa} \cdot \mathrm{m}^{1 / 2}$ to $3.85 \mathrm{MPa} \cdot \mathrm{m}^{1 / 2}$ due to the difference in coating thickness, carbide size and coating processing method. Chivavibul et al. [121] derived that the fracture toughness of HVOF WC-Co coating with 15 $\mathrm{wt} \% \mathrm{Co}$ was in the range of $3 \mathrm{MPa} \cdot \mathrm{m}^{1 / 2}$ to $4 \mathrm{MPa} \cdot \mathrm{m}^{1 / 2}$ for different grain sizes; Demidova et al. [18] determined the fracture toughness of HVOF WC-10Co4Cr coating to be $K_{I C}=1.21 \mathrm{MPa} \cdot \mathrm{m}^{1 / 2}$

Meanwhile, based on the experimental data, when the volume fraction of $\delta$-WC phase is less than $80 \%$, the fracture toughness of the two-phase composite coating mainly depends on the fracture toughness of $\alpha$-Co phase [105]. As a result, the variation of the calculated temperature-dependent fracture toughness of WC-10Co4Cr coating/1018 low carbon steel substrate system is similar to that of ductile $\alpha$-Co phase, and the estimated fracture toughness increases exponentially with temperature. The fracture toughness of the coating/substrate system has a great increment from $2.1567 \mathrm{MPa} \cdot \mathrm{m}^{1 / 2}$ to $10.815 \mathrm{MPa} \cdot \mathrm{m}^{1 / 2}$ with increasing temperature. This result partially consists with the temperature-dependent fracture toughness of several types of WC-Co based alloys, as shown in Figure 3-13.

Using the basic mixture method according to Eq. (3-13), the fracture toughness of WC-10Co4Cr coating/1018 low carbon steel substrate system is obtained and plotted in 
Figure 3-18. For the two-phase composite coating, the fracture toughness calculated from the crystal-structure-based model using this mixture method, is greater than that from the model using the unconstrained mixture method at low temperatures (below $280 \mathrm{~K}$ ), and this relationship is reversed for relative high temperatures (above $280 \mathrm{~K}$ ). Because the effect of $\delta$-WC phase on fracture toughness is larger in basic mixture method than in unconstrained mixture method. When compared with the experimental data, the curve obtained from the unconstrained mixture method would describe the relationship between the fracture toughness and temperature more precisely [105], because the trapping, bowing, and bridging behaviors of the composite coating are all considered in this model. The basic mixture method estimates the integrated fracture toughness only based on the volume fraction of each phase, however, the real situation is more complex than that. As discussed earlier, the validity of the unconstrained mixture method in the present research has been proved with good accuracy on the fracture toughness estimation of WC-Co composite. 


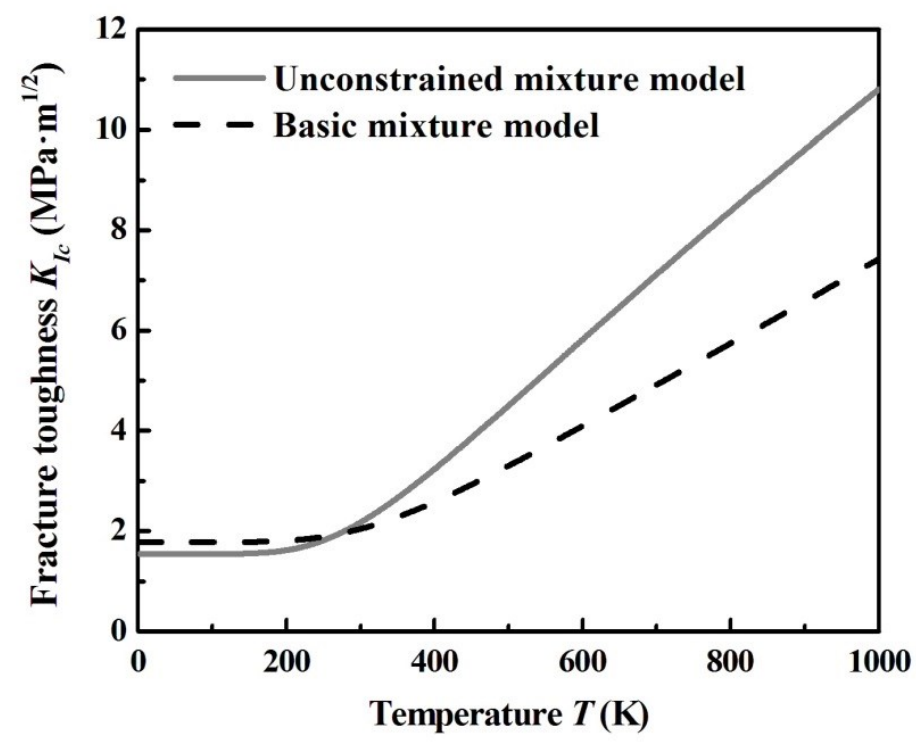

Figure 3-18. Temperature-dependent fracture toughness of WC-10Co4Cr coating/1018 low carbon steel substrate system based on two mixture models.

As discussed previously, some parameters required in the fracture toughness estimation of the composite coating are obtained from literature. The surface energy data of $\alpha$-Co phase derived from several different approaches are consistent, but the surface energy of $\delta$-WC phase varies from $2 \mathrm{~J} / \mathrm{m}^{2}$ to $3.7 \mathrm{~J} / \mathrm{m}^{2}[110,113,114]$. The surface energies of both phases exert an obvious effect on the estimated fracture toughness of the composite coating, since at low temperatures $(600 \mathrm{~K}$ for $\delta$-WC phase), the fracture toughness of $\delta$-WC coating is determined by the work done by elastic component in terms of surface energy, $\gamma_{s}$.

As illustrated in Figure 3-19, the fracture toughness of single-phase $\delta$-WC coating and WC-10Co4Cr composite coating increase by $0.6065 \mathrm{MPa} \cdot \mathrm{m}^{1 / 2}$ and $0.2495 \mathrm{MPa} \cdot \mathrm{m}^{1 / 2}$, respectively, with the increase in surface energy. According to these calculation results, the 
surface energy, $\gamma_{s}$, of $\delta$-WC phase is crucial for the fracture toughness estimation of $\delta$-WC coating and $\mathrm{WC}-10 \mathrm{Co} 4 \mathrm{Cr}$ composite coating at low temperatures.

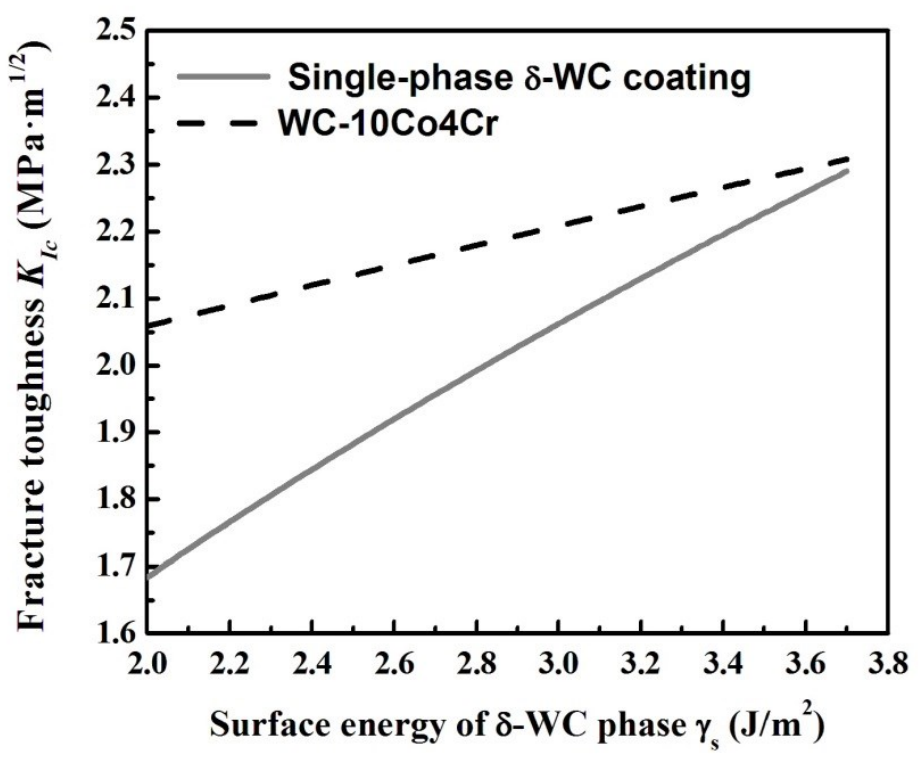

Figure 3-19. Relationship between the surface energy, $\gamma_{s}$, of $\delta$-WC phase and the fracture toughness.

In addition, the ratio of surface energy to $(\mathrm{P}-\mathrm{N})$ barrier energy, $\gamma_{S} / U_{P-N}$, is also a critical factor for fracture resistance evaluation of materials. The ratio of $\gamma_{S} / U_{P-N}$ describes the material's tendency to crack propagation with respect to the difficulty of dislocation movement [14], and it is larger for hard phases such as $\delta$-WC phase than for soft phases like $\alpha$-Co phase. As shown in Figure 3-20, the ratio of $\gamma_{S} / U_{P-N}$ can be associated with the material's fracture toughness, and the materials with higher ratio of $\gamma_{S} / U_{P-N}$ have better fracture resistance. 


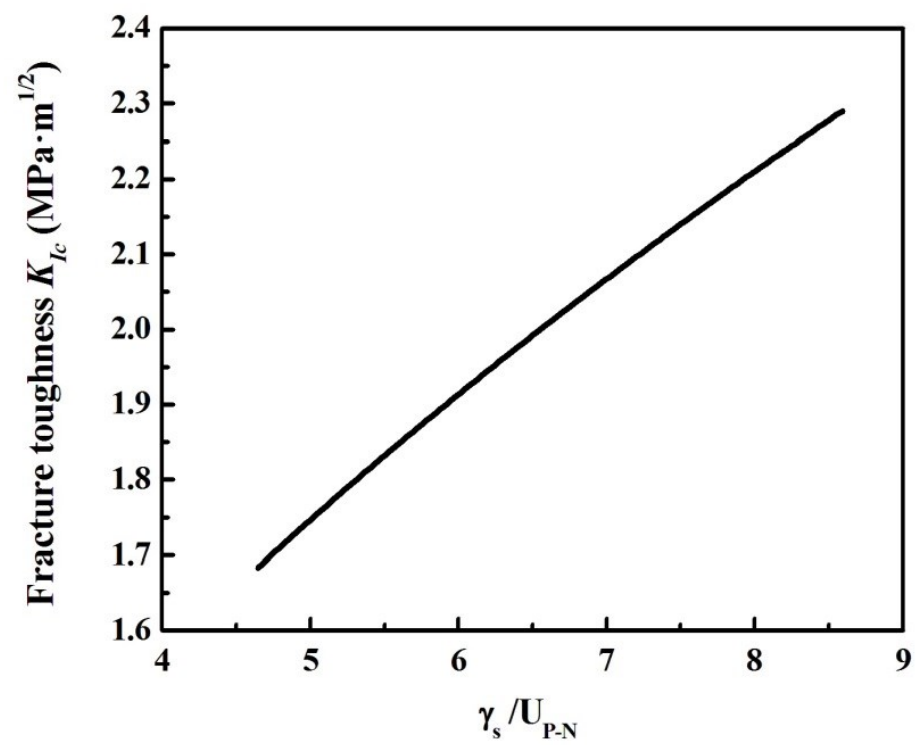

Figure 3-20. Relationship between $\gamma_{S} / U_{P-N}$ and the fracture toughness of $\delta$-WC coating.

\subsection{Closing Remarks}

In this chapter, the composition of selected HVOF WC-10Co4Cr composite coating is analyzed, the two main phases are the brittle $\delta$-WC phase and ductile $\alpha$-Co phase. The microstructure and the slip system of these two phases are discussed based on the phase diagrams. It is shown that the Burgers vector of $1 / 3[1 \overline{2} 10]$ on prismatic slip plane $\{10 \overline{1} 0\}$ is responsible for the slip system of $\delta$-WC phase, and the Burgers vector of $1 / 2[110]$ on the slip plane $\{111\}$ is responsible for the slip system of $\alpha$-Co phase.

Based on the temperature-dependent fracture toughness model developed at microscopic level for bulk materials [14], and the median/radial crack propagation model proposed by Lawn et al. [15] and Cook et al. [16], an analytical model for the temperature-dependent 
fracture toughness estimation of $\mathrm{WC}-10 \mathrm{Co} 4 \mathrm{Cr}$ coating/1018 low carbon steel system is derived, by substituting the area of plastic zone in the composite coating for that in bulk materials under indentation loading. The dislocation density variation with the indentation load is integrated into the fracture toughness calculation using the model developed in Durst's work [17]. The concept of the radial cracks generated in the coating and fully developed during the unloading process can be applied to the fracture toughness estimation of the brittle coating/ductile substrate system, and the residual stress in terms of the energy stored in plastic zone during the loading process is the major driving force for the propagation of radial cracks.

The fracture toughness of the composite coating/substrate system remains unchanged until the temperature reaches a critical value. Then the fracture toughness increases rapidly and rises in an exponential manner. The critical temperatures for single $\delta$-WC and $\alpha$-Co coatings are $600 \mathrm{~K}$ and $200 \mathrm{~K}$, respectively. This is mainly determined by the relationship between the work done by elastic and plastic deformations.

The fracture toughness of $\mathrm{WC}-10 \mathrm{Co} 4 \mathrm{Cr}$ coating/1018 low carbon steel system is calculated by using the unconstrained mixture model which has been verified by comparing the simulation results with the experimental results. The estimated fracture toughness has an increment from $2.1567 \mathrm{MPa} \cdot \mathrm{m}^{1 / 2}$ to $10.815 \mathrm{MPa} \cdot \mathrm{m}^{1 / 2}$ over a temperature range from $298 \mathrm{~K}$ to $1000 \mathrm{~K}$. The room temperature fracture toughness and the experimental data 
obtained by other researchers are in the same order of magnitudes. The fracture toughness estimated using the analytical model developed in this chapter is independent of indentation load. 


\section{Chapter 4. Temperature-dependent Fracture Toughness Models Based on Microcrack Formation Theory}

In Chapter 3, the plastic term of strain energy release rate in terms of the work done by plastic deformation is described by the temperature-dependent model which is based on the Arrhenius-type equation and rate controlling theory. For a brittle coating/ductile substrate system under indentation loading, the developed model can be expressed as:

$$
\begin{gathered}
J_{d}=J_{0} \exp \left[-\frac{b^{2}\left(\gamma_{F}+U_{P-N}\right)}{k T}\right] \\
J_{0}=2 \rho h t\left(\frac{k T}{b^{2}}\right)
\end{gathered}
$$

where $h$ is the dimension of plastic zone in the coating, $\rho$ is dislocation density and $t$ is coating thickness. Based on this theory, microcrack-formation-based models are developed to evaluate the temperature-dependent fracture toughness of $\mathrm{WC}-10 \mathrm{Co} 4 \mathrm{Cr}$ coating/1018 low carbon steel substrate system in this chapter. The calculations utilize the experimental data obtained from the indentation tests performed on the same coating/substrate system in Demidova's work [18]. 


\subsection{Microcracks in Brittle Coating}

In Eq. (4-1), the temperature-dependent fracture toughness can be evaluated by calculating the total dislocation energy stored in the plastic zone, which is responsible for radial crack propagation during the unloading process of indentation.

\subsubsection{Microcrack formation}

For 2D radial crack extension, as discussed previously, in the unloading process, numerous microcracks are generated from the each corner of indentation impression and merge together as radial cracks under tensile residual stresses, and the radial cracks extend along the indentation diagonals, as shown in Figure 4-1.

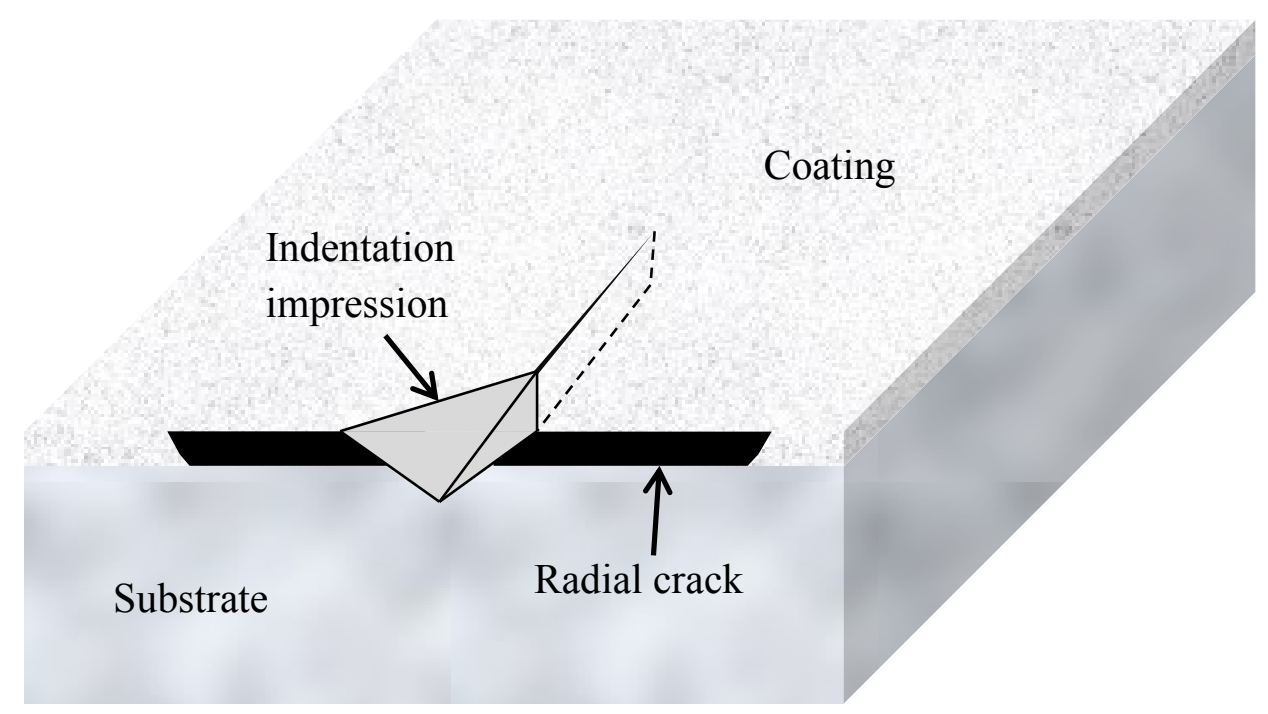

Figure 4-1. Schematic illustration of radial cracks in a brittle coating under Vickers indentation. 
Ortiz [19] indicated that, in brittle materials, the microcracks can be assumed to be evenly spaced and have the same length during the crack extension. As shown in Figure 4-2, microcracks in the crack tip zone have a characteristic length, $L$, in the tensile stress configuration. Although there are gaps between each microcrack, the size of the gaps is too small to take into account when compared with the length of microcracks. Meanwhile, the microcrack opening displacement, $\zeta$, increases from 0 to $\delta$ during the crack propagation process, since the microcracks in the front of the macrocrack tip become the new crack tip one after another when they are merged into the macrocrack. Therefore, the crack tip opening displacement (CTOD), $\delta$, could be used to measure the total growth of a microcrack in tensile direction. Under this assumption, the dislocation movement could be associated with the crack propagation process.

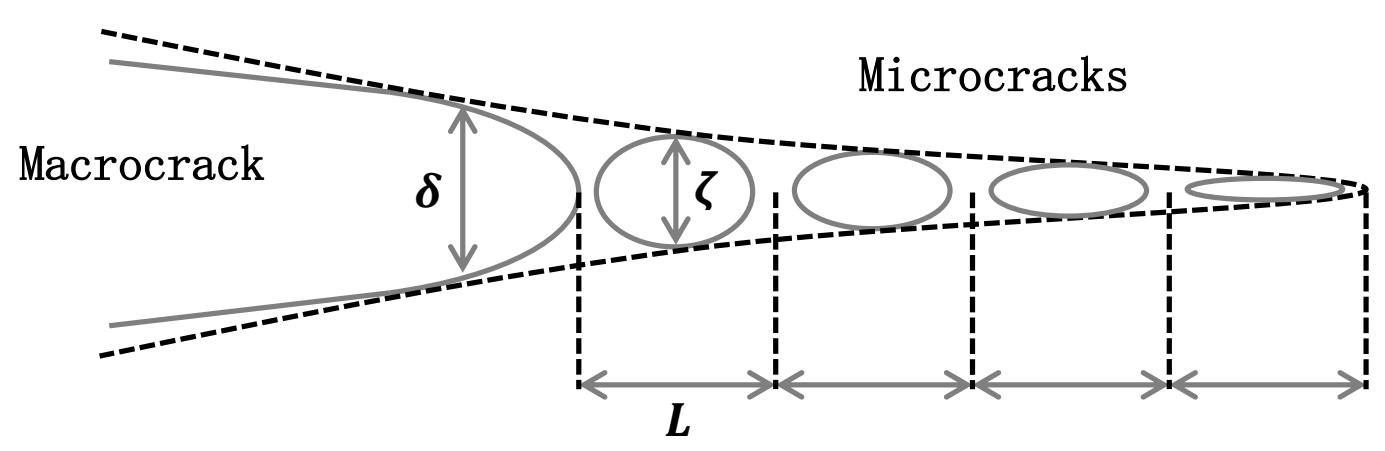

Figure 4-2. Schematic illustration of the crack tip of macrocrack and microcrack formation in brittle materials. 
The amount of crack tip opening displacement, $\delta$, for brittle materials can be assessed based on material's properties, and one of the general expressions for CTOD calculation is given by Eq. (4-2) [125], and the validity of Eq. (4-2) for WC-Co composite has been proved in Murray's work [126]. Thus, the total growth of a microcrack in tensile direction, which approximately equals to the size of $\operatorname{CTOD}(\zeta \approx \delta)$, can be estimated as:

$$
\delta=\frac{G_{e}}{2 \tau_{c}}
$$

where $G_{e}$ is strain energy release rate, $\tau_{c}$ is yield stress of the coating and determined to be 210MP [127] for WC-10Co4Cr composite coating.

Fracture toughness is usually estimated based on the applied load and crack length data which can be obtained from indentation tests. The length of radial cracks is always a critical factor in the fracture toughness evaluation as expressed by Eq. (4-3a). However, Moradkhani et al. [128] argued that, for 2D radial crack propagation, the fracture toughness of brittle materials can also be calculated based on indentation load with crack area profile instead of crack length. In their work, the crack length was replaced by the total crack area and average depth of microcracks, as described by Eq. (4-3b).

$$
\begin{gathered}
K_{c}=\xi_{r}^{R}\left(\frac{E}{H}\right)^{1 / 2} \cot (\psi)^{3 / 2}\left(\frac{P}{c^{3 / 2}}\right) \\
K_{c}=\xi_{r}^{R}\left(\frac{E}{H}\right)^{1 / 2} \cot (\psi)^{3 / 2}\left(t^{3 / 2} \frac{P}{A^{3 / 2}}\right)
\end{gathered}
$$

where $c$ is the average length of radial cracks, $A$ stands for the area that covers all of the microcracks generated in the deformation zone and $t$ is the average depth of microcracks or 
the thickness of the coating area where microcracks are initiated during indentation tests (for through-thickness cracks). Based on their discussion, the crack area profile can be used to investigate the fracture toughness of brittle materials.

\subsubsection{Dislocation movement of atoms}

The microcracks formed in the composite coating are caused by the dislocation movement of atoms in the deformation zone. The magnitude and direction of dislocation movement can be described by Burgers vector. In the previous chapter, the temperature-dependent fracture toughness was evaluated using the dislocation energy stored in the deformation zone, as expressed by Eq. (4-1). The maximum work done by plastic deformation per unit area of crack extension, $J_{0}$, is described by the dislocation density, $\rho$, the dimension of plastic zone, $h$, and the thermal activation energy for per unit length of dislocation motion, $k T / b^{2}$. Therefore, the fracture toughness of brittle materials under indentation loading can also be evaluated with microcrack density and the energy for microcrack formation.

The total amount of energy variation during the fracture process can be described at microscopic level using strain energy release rate, surface energy, energy barrier and stacking fault energy. The dislocation movement of atoms in this process could be depicted through Burgers vector and the slip system of the material under certain load type. As shown in Figure 4-3, the microcracks generated under tensile stresses can be described using the movement of atoms. When deformation occurs in the material, the atoms in certain area may 
deviate from their equilibrium position. During the atomic deviation process, energy change takes place in the bonds between atoms. The bonds are broken when the force or the energy exceeds a critical value. Microcracks are initiated and propagate due to the atomic deviation and the absence of connection between atoms. Although this description is based on 2D profile and much simpler than real situation, it can be applied to microcrack formation and the fracture toughness model at micromechanics scale.

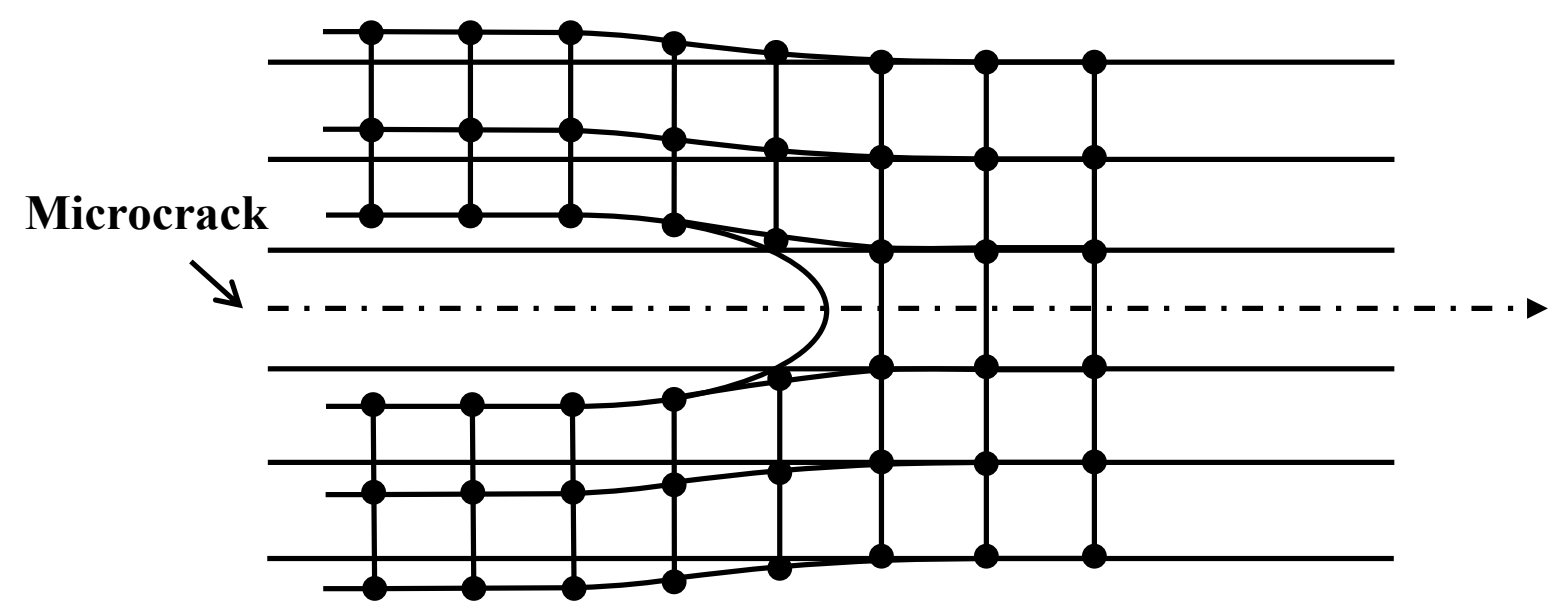

Figure 4-3. Schematic illustration of microcrack formation and the movement of atoms.

According to this description, the size of microcracks generated during crack propagation and the magnitude of dislocation movement in terms of Burgers vector can be approximately related by a parameter $m$ with $m b=\delta$, where $\delta$ is the crack tip opening displacement, $b$ is Burgers vector. The similar description for the relationship between CTOD and Burgers vector was proposed in literature [129]. 


\subsection{Model Development}

The model developed in this chapter is based on the fracture toughness model in Hu's work [9] which takes the interface sliding and substrate effect into the calculation, and the Arrhenius-type equation introduced previously.

\subsubsection{Energy release for microcrack propagation}

As discussed previously, for a brittle coating deposited on a ductile substrate, $\mathrm{Hu}$ and Evans [9] proposed that the energy release rate, $G_{e}$, for the propagation of cracks whose lengths were much greater than the coating thickness, $t$, was determined by the coating thickness rather than the crack length. Furthermore, they discussed that for steady-state cracking on coatings, the total strain energy change, $\Delta U_{e}$, was determined by the coating thickness and independent of crack profiles, and the energy release rate, $G_{e}$, can be derived from the total strain energy change, $\Delta U_{e}$, where $\Delta U_{e}$ and $G_{e}$ are given by:

$$
\begin{aligned}
& \Delta U_{e}=\frac{\pi F\left(\sum\right) \sigma^{2} t^{2}}{E^{c}} \\
& \Delta U_{e}=\int_{0}^{t} G_{e}(z) \mathrm{d} z
\end{aligned}
$$

or

$$
G_{e}=\frac{\Delta U_{e}}{t}
$$

where $\Sigma$ is the elastic modulus ratio with $\Sigma=E^{c} / E^{s}, E^{c}$ and $E^{s}$ are the Young's modulus of the brittle coating and ductile substrate, respectively. $F(\Sigma)$ is a function about the elastic modulus ratio $\Sigma$ and can be defined as [130],

$$
F(\Sigma)=\int_{0}^{1} \alpha g(\alpha, \Sigma) \mathrm{d} \alpha
$$


where $\mathrm{g}(\alpha, \Sigma)$ is a function of the ratio of crack depth, $d$, to coating thickness, $t$, and $\alpha=d / t$. Some values of the elastic modulus related function $F(\Sigma)$ were obtained by Hu [9] and are given in Table 4-1:

Table 4-1. Values of function $F(\Sigma)$ corresponding to different elastic modulus ratios [9]

\begin{tabular}{|c|c|}
\hline$\Sigma$ & $F(\Sigma)$ \\
\hline 4 & 0.79 \\
\hline 3 & 0.75 \\
\hline 2 & 0.70 \\
\hline 1 & 0.62 \\
\hline $1 / 2$ & 0.57 \\
\hline $1 / 3$ & 0.54 \\
\hline
\end{tabular}

The elastic modulus ratio $\Sigma$ for the composite coating is calculated to be $1.24\left(E_{c} / E_{s}=\right.$ $310 \mathrm{GPa} / 250 \mathrm{GPa}=1.24)$. Then the value of function $F(\Sigma)$ can be determined as 0.64 based on the graph of $F(\Sigma)$ versus the elastic modulus ratio $\Sigma$, as illustrated in Figure 4-4. It is seen that Figure 4-4(b) shows some values of function $F(\Sigma)$ corresponding to elastic modulus ratio close to 1.24 , the four significant digits are needed to distinguish the difference between the results. 


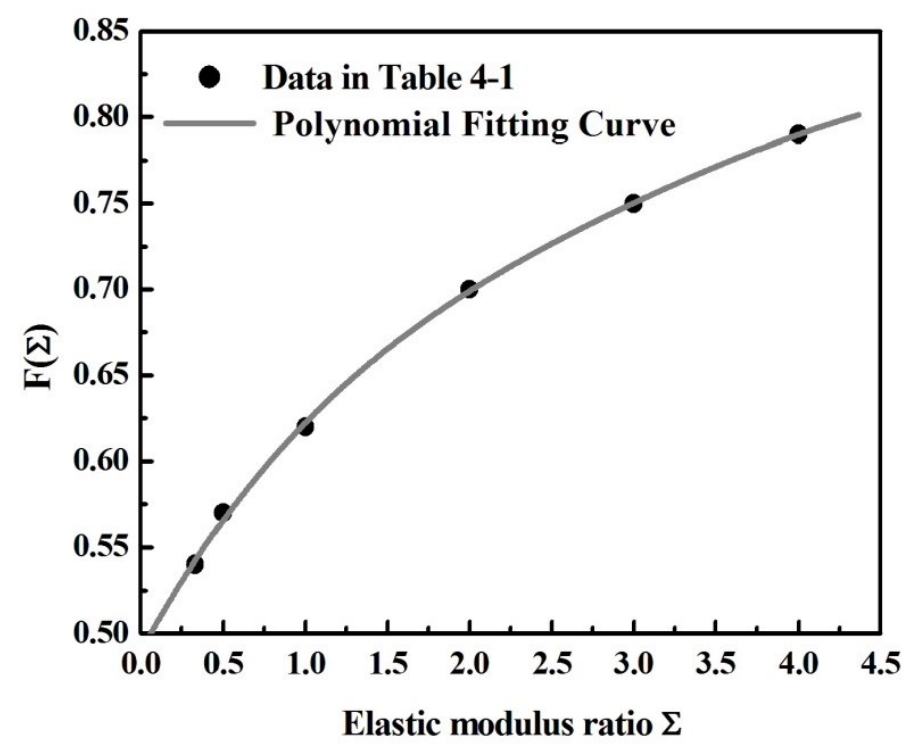

(a)

\begin{tabular}{|r|r|r|}
\hline 576 & 1.2323 & 0.64381 \\
\hline 577 & 1.2345 & 0.644 \\
\hline 578 & 1.2367 & 0.64419 \\
\hline 579 & 1.2389 & 0.64438 \\
\hline 581 & & \\
\hline 582 & 1.2433 & 0.64476 \\
\hline 583 & 1.24551 & 0.64495 \\
\hline 584 & 1.24771 & 0.64514 \\
\hline
\end{tabular}

(b)

Figure 4-4. $F(\Sigma)$ versus the elastic modulus ratio $\Sigma$ : (a) graph, (b) polynomial fitting data.

The total stress, $\sigma$, is defined as:

$$
\sigma=\sigma_{0}+\sigma_{\infty} \frac{E^{c}}{E^{s}}
$$

where $\sigma_{\infty}$ is the applied tension, and $\sigma_{0}$ is the residual stress in the coating. Finally, the stress intensity factor of the coating/substrate system can be described as: 


$$
K_{s s}=\sqrt{G_{e} E^{c}}=\sigma \sqrt{\pi t F\left(\sum\right)}
$$

For through-thickness cracks, however, the interface sliding in certain areas between the coating and substrate has to be considered. In Hu's work [9], for coatings whose thickness is much smaller than that of substrates suffer a constant shear stress, $\tau$, in the interface sliding region, as shown in Figure 4-5. The shear stress, $\tau$, can be determined by the yield strength of the substrate material, and this assumption is valid when either sliding or yielding occurs in the coating near the interface.

\section{Through-thickness crack}

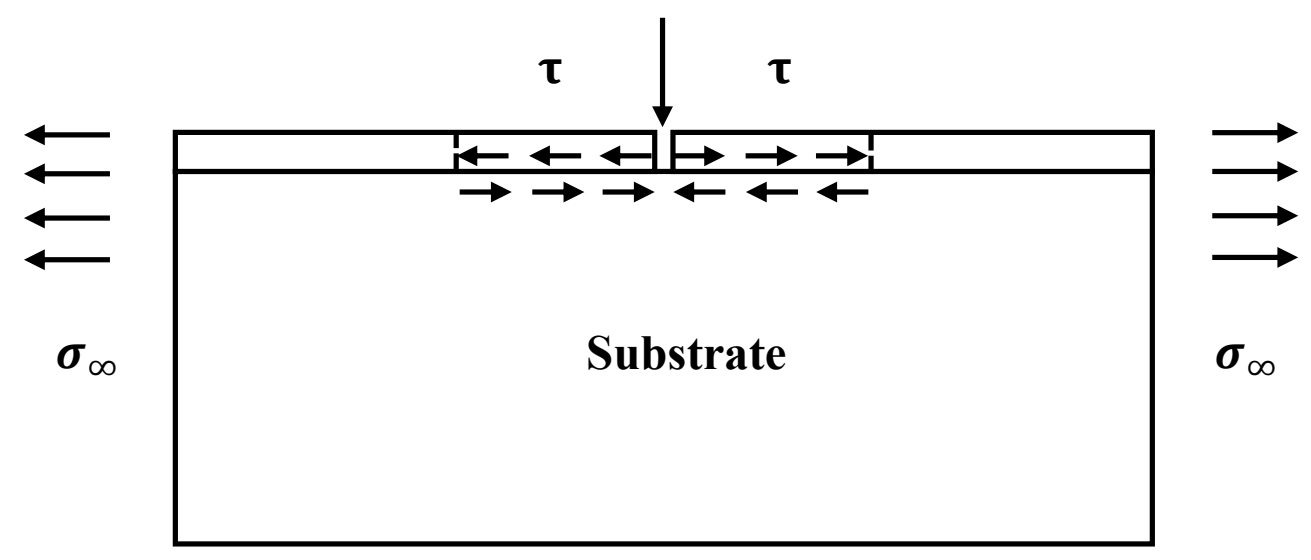

Figure 4-5. A constant shear stress at the coating/substrate interface.

Therefore, the strain energy change in Eq. $(4-4 c)$ is replaced by the total energy variation, $\Delta U$, which should include the energy dissipation caused by the interface sliding and can be described as:

$$
\Delta U=\Delta W+\Delta W_{d}+\Delta U_{s}+\Delta U_{e}
$$


where $\Delta W$ is the total work done by the applied load, $\Delta W_{d}$ is the dissipated energy caused by interface sliding, $\Delta U_{s}$ is the strain energy variation due to interface sliding, and $\Delta U_{e}$ is the energy change for steady-state cracking. These terms were derived in Evans' [39] and Marshall's work [40] in terms of fracture mechanics:

$$
\begin{aligned}
& \Delta W=-\frac{\sigma^{3} t^{2}}{\tau E^{c}} \\
& \Delta W_{d}=\frac{\sigma^{3} t^{2}}{3 \tau E^{c}} \\
& \Delta U_{s}=\frac{\sigma^{3} t^{2}}{3 \tau E^{c}}
\end{aligned}
$$

where $E^{c}$ is the Young's modulus of the brittle coating. According to Eq. (4-4) to Eq. (4-9), the total energy change, $\Delta U$, and the strain energy release rate, $G_{e}$, are given by:

$$
\begin{aligned}
& \Delta U=-\frac{\sigma^{2} t^{2}}{E^{c}}\left(\frac{\sigma}{3 \tau}+\pi F\left(\sum\right)\right) \\
& G_{e}=-\frac{\Delta U}{t}=\frac{\sigma^{2} t}{E^{c}}\left(\frac{\sigma}{3 \tau}+\pi F(\Sigma)\right)
\end{aligned}
$$

and the stress intensity factor of the coating/substrate system can be described as:

$$
K_{s s}=\left[\sigma^{2} t\left(\frac{\sigma}{\sqrt{3} Y}+\pi F\left(\sum\right)\right)\right]^{1 / 2}
$$

where $Y$ is the yield strength of the substrate and $Y=\sqrt{3} \tau$ in terms of Von Mises yield criterion [41]. The yield strength of 1018 low carbon steel is $380 \mathrm{MPa}$ [131]. From this model, the strain energy release rate, $G_{e}$, at room temperature is $G_{e}=13.4390 \mathrm{~J} / \mathrm{m}^{2}$ for WC-10Co4Cr coating/1018 low carbon steel substrate system. This result is very close to the calculated strain energy release rate obtained from the model developed in Chapter 3. 
The formation of microcracks is related to the dislocation motion of atoms, and the magnitude of dislocation motion in terms of Burgers vectors can be used to describe the dimension of microcracks. Based on the fracture toughness model for mode I crack propagation, given by $\mathrm{Hu}[9]$, which takes the coating/substrate interface effect into account, as demonstrated by Eq. (4-10) and Eq. (4-11), the strain energy release rate and fracture toughness of the coating/substrate system can be calculated for room temperature. The key feature of this new model is that it relates the strain energy release rate, $G_{e}$, in Hu's work to the temperature variation, and describes the temperature-dependent strain energy release rate, $G_{s S}$, based on the rate controlling theory. Then the temperature-dependent fracture toughness of the composite coating/substrate system can be obtained.

Using the crack propagation model discussed above and the micro-level fracture toughness model which relates the strain energy release rate to the dislocation movement, the temperature-dependent fracture toughness model in terms of strain energy release rate for brittle coating/ductile substrate systems under indentation loading can be proposed :

$$
\begin{gathered}
G_{s s}=G_{0} m A \rho_{c} \exp \left(-\frac{\frac{G_{e}}{n}\left(\frac{\delta}{m}\right)^{2}}{k t}\right) \\
G_{0}=\frac{k T}{\left(\frac{\delta}{m}\right)^{2}}
\end{gathered}
$$


where $k$ is Boltzmann constant, $T$ is absolute temperature. $G_{e}$ is the strain energy release rate at room temperature, obtained from Eq. (4-10b), and $n$ is a scale-linking parameter that relates the strain energy release rate for crack extension to the micro-level energy, $G_{m}$, that must be overcome by the dislocation motion of atoms. The strain energy release rate, $G_{e}$, describes the energy dissipation during the crack propagation process, and only if the dislocation motion can overcome the energy barrier, will microcracks be generated in the plastic deformation zone. This assumption is very similar to that of the relationship between the size of microcracks in terms of CTOD, $\delta$, and the magnitude of Burgers vector, $b$, with $m b=\delta$, as discussed in last section. Therefore, the relationship between the micro-level energy barrier, $G_{m}$, and the strain energy release rate, $G_{e}$, can be described as $n G_{m}=G_{e}$. The significance of the parameter $n$ is to confirm that the energy release rate calculated from Eq. (4-12a) at room temperature is the same as $G_{e}$, and the value of $n$ is determined based on the experimental data of radial crack profiles under different indentation loads, which were obtained by Demidova [18].

\subsubsection{Substrate effects}

As analyzed in the previous sections, Eq. (4-12) is based on microcrack formation and the Arrhenius-type equation, which associates micromechanics and thermodynamics with fracture behavior. In Eq. (4-12a), $G_{0} m A \rho_{c}$ can be interpreted as the maximum plastic work per unit area of crack extension, where $G_{0}$ stands for the energy needed to overcome by the atoms moving a single characteristic length in terms of Burgers vector, $\rho_{c}$ is microcrack 
areal density, and $A$ is the total area of plastic deformation zone with microcracks, which can be considered as the radial crack area, as shown in Figure 4-1, because the microcracks which merge together to form radial cracks are generated due to the residual stresses stored in the plastic zone. As a result, this plastic zone can be determined as the radial crack area and expressed as:

$$
A=2 c t
$$

where $c$ is the average length of radial cracks.

The microcrack areal density, $\rho_{c}$, for coating/substrate systems was given by Liu and Nairn [132] as:

$$
\rho_{c}=\frac{1}{t D}
$$

where $t$ is coating thickness and $D$ is crack spacing, which can be interpreted as the reciprocal of liner density. The crack spacing, $D$, for coating/substrate systems is determined by the applied load and the material properties of the system. The expression of crack spacing, $D$, for brittle coating/ductile substrate systems was derived by Nagl and Evans [133]:

$$
D=\frac{2 t \sigma}{\tau_{y}}
$$

where $\sigma$ is applied stress in the coating, $\tau_{y}$ is the shear yield stress of the substrate, and $\tau_{y}=Y / \sqrt{3}$ based on Von Mises yield criterion [41]. 
As discussed above, the applied stress which is responsible for crack extension should be the residual stresses stored in the plastic zone during the loading period, and the equation for the residual stresses under indentation loads was given by Chiang et al. [134]:

$$
\sigma=p\left\{\frac{3\left[\ln \left(r / a_{c}\right)+1 / 2\right]}{1+3 \ln (f)}\right\}-1-\frac{1}{2\left(r / a_{c}\right)^{3}} \quad(f>r / a>1)
$$

where $f$ is the ratio between the radius of plastic zone and the half diagonal of indentation impression, which is discussed in previous chapter and calculated as 4.1162 for $\mathrm{WC}-10 \mathrm{Co} 4 \mathrm{Cr}$ composite coating, $r$ is the distance from cavity center and $a_{c}$ is the half diagonal of indentation impression. By assuming the stress in plastic zone to be a constant, Eq. (4-16) can be changed into:

$$
\sigma=p\left\{\frac{3[\ln (f)+1 / 2]}{1+3 \ln (f)}\right\}-1-\frac{1}{2(f)^{3}}
$$

In the residual stress model developed by Chiang et al. [134], the parameter $p$ stands for the indentation pressure, which is obtained from Eq. (4-18):

$$
p=\frac{P}{\left(\sqrt{2} a_{c}\right)^{2}}
$$

where $P$ is the indentation load and $a_{c}$ is the half diagonal of indentation impression. By recognizing that the expressions of indentation pressure and Vickers hardness have the same form, two approaches are applied to the indentation pressure and the fracture toughness calculation of the composite coating/substrate system.

In Demidova's work [18], the substrate effect on the fracture toughness of the system was taken into account by introducing composite hardness. For estimating the fracture toughness 
of a brittle coating/ductile substrate system using the through-thickness crack profiles, as shown in Figure 3-9, the fracture behavior of the brittle coating is substantially influenced by the properties of substrate material, and the effect of substrate can be described by the coating/substrate composite hardness in terms of indentation pressure. The composite Vickers hardness equation of brittle coating/ductile substrate systems was developed by Ichimura [135] and can be expressed as:

$$
H_{m}=3\left(\frac{t}{2 a_{c}}\right)\left(\frac{H_{c}}{E_{c}}\right)^{1 / 2} \cdot\left(H_{c}-H_{s}\right)(\tan \psi)^{1 / 3}+H_{s}
$$

where $H_{m}$ is the composite Vickers hardness, $\Psi$ is the half-angle of the indenter, $E_{c}$ is the elastic modulus of the composite coating, $H_{c}$ and $H_{s}$ are the Vickers hardness of the composite coating and substrate, respectively. The values of the composite hardness for each indentation load are calculated and presented in Table 4-7.

However, both the indentation pressures calculated from Eq. (4-18) and Eq. (4-19) can reflect the effect of ductile substrate on the system. Therefore, the two approaches are applied to the indentation pressure estimation, and all of the parameters required are determined. The temperature-dependent fracture toughness for the composite coating/substrate system is calculated using these two approaches based on the data obtained in Demidova's experimental work [18]. 


\subsection{Results of Fracture Toughness}

The temperature-dependent fracture toughness for the composite coating/substrate system is calculated based on the model developed in this chapter using the basic indentation pressure approach and the composite hardness approach. The data of radial crack length and indentation impression diagonal, which were obtained from Demidova's work under indentation loading [18], are applied to the calculations.

\subsubsection{Basic indentation pressure approach}

Using the basic indentation pressure approach (Eq. (4-18)), the two parameters, $m$ and $n$, need to be determined before the fracture toughness calculation. The parameter $m$, which relates the size of microcracks in terms of CTOD, $\delta$, to the magnitude of Burgers vector, $b$, can be determined by comparing the ratio $\delta / m$ with the Burgers vector of dislocation movement. In $2 \mathrm{D}$ crack problems, the ratio $\delta / m$ should be approximately equal to the magnitude of dislocation motion in terms of Burgers vector, since the microcracks result from the movement of dislocations, as shown in Figure 4-3. The Burgers vector magnitudes of $\delta$-WC phase and $\alpha$-Co phase in the $\mathrm{WC}-10 \mathrm{Co} 4 \mathrm{Cr}$ composite coating are calculated to be $0.2906 \mathrm{~nm}$ and $0.2519 \mathrm{~nm}$, respectively. The ratio $\delta / m$ should be between the Burgers vector magnitudes of $\delta$-WC phase and $\alpha$-Co phase.

The crack tip opening displacement is determined based on the basic properties of the coating material and independent of indentation load, the CTOD of WC-10Co4Cr composite coating 
is calculated as $31.9982 \mathrm{~nm}$ using Eq. (4-2). According to the unconstrained mixture model given in Eq. (3-14), the mixture Burgers vector magnitude of the two phases could be approximately estimated as $0.2701 \mathrm{~nm}$, corresponding to the parameter $m=118.468$. Although this specific value, 118.468 , is approximated via the unconstrained mixture model, it could rightly relate the size of microcracks to the magnitude of the Burgers vector.

The scale-linking parameter $n$ which relates the micro-level energy barrier, $G_{m}$, to the strain energy release rate, $G_{e}$, can be determined based on the crack length and indentation impression data summarized in Table 4-2 and Table 4-3, which were obtained from Demidova's experimental work [18]. The parameter $n$ has several functions in this model. First, it connects the microscopic energy to macroscopic energy. Second, in order to compare the high-temperature fracture toughness of the composite coating/substrate system obtained from different models, the parameter $n$ is introduced to confirm that the room temperature energy release rate calculated from Eq. (4-12a) is the same as the energy release rate, $G_{e}$, estimated from Hu's model [9]. Third, it is used to minimize the load effect at room temperature, which is similar to the function of crack shape influence factor.

As shown in Table 4-2, under the same indentation load, the average radial crack length $c$ (mean value of the four radial crack lengths in one test) varies within a large range. Therefore, the average crack length $c_{\text {avg }}$ was calculated based on a group of crack length data obtained under the same indentation load at room temperature in Demidova's work [18]. These crack 
geometry data are used to determine the microcrack density at room temperature. The average indentation diagonal, $2 l$, and the indentation pressure, $p$, calculated using Eq. (4-18) under different indentation loads, $P$, are presented in Table 4-3. The microcrack density, $\rho_{c}$, the parameter $n$, the strain energy release, $G_{S S}$, and the fracture toughness, $K_{S S}$, at room temperature for each indentation diagonal value, are calculated using MATLAB 2010b and the results are presented in Table 4-4. The detailed calculations are given in the Appendix. The variations of indentation pressure, $p$, the parameter $n$, and microcrack density, $\rho_{c}$, with indentation load are discussed below. 
Table 4-2. Average radial crack length $c$ under each indentation load [18]

\begin{tabular}{|c|c|c|c|}
\hline $\begin{array}{l}\text { Indentation } \\
\text { mark \# }\end{array}$ & Load $P(\mathrm{~N})$ & $\begin{array}{l}\text { Average radial crack } \\
\text { length } c(\mu \mathrm{m})\end{array}$ & Average value $c_{a v g}(\mu \mathrm{m})$ \\
\hline 1 & \multirow{5}{*}{196} & 433.0 & \multirow{5}{*}{497.700} \\
\hline 2 & & 474.5 & \\
\hline 3 & & 508.5 & \\
\hline 4 & & 521.5 & \\
\hline 5 & & 551.0 & \\
\hline 6 & \multirow{6}{*}{294} & 581.0 & \multirow{6}{*}{622.330} \\
\hline 7 & & 610.5 & \\
\hline 8 & & 621.5 & \\
\hline 9 & & 641.5 & \\
\hline 10 & & 646.5 & \\
\hline 11 & & 633.0 & \\
\hline 12 & \multirow{4}{*}{392} & 653.5 & \multirow{4}{*}{686.625} \\
\hline 13 & & 678.5 & \\
\hline 14 & & 684.0 & \\
\hline 15 & & 730.5 & \\
\hline 16 & \multirow{4}{*}{490} & 696.0 & \multirow{4}{*}{727.875} \\
\hline 17 & & 715.5 & \\
\hline 18 & & 718.5 & \\
\hline 19 & & 781.5 & \\
\hline 21 & \multirow{4}{*}{720} & 872.5 & \multirow{4}{*}{859.100} \\
\hline 22 & & 868.5 & \\
\hline 23 & & 785.5 & \\
\hline 24 & & 864.0 & \\
\hline
\end{tabular}


Table 4-3. Average indentation diagonal $2 l$ and indentation pressure $p$ under each applied load for the basic indentation pressure approach

\begin{tabular}{|c|c|c|c|}
\hline $\begin{array}{l}\text { Indentation } \\
\text { mark \# }\end{array}$ & Load $P(\mathrm{~N})$ & $\begin{array}{l}\text { Average indentation } \\
\text { diagonal } 2 l(\mu \mathrm{m})\end{array}$ & $\begin{array}{c}\text { Indentation pressure } p \\
(\mathrm{GPa})\end{array}$ \\
\hline 1 & \multirow{3}{*}{196} & 616.6 & 1.0310 \\
\hline 2 & & 737.5 & 0.7207 \\
\hline 3 & & 628.5 & 0.9924 \\
\hline 4 & \multirow{3}{*}{294} & 813 & 0.8896 \\
\hline 5 & & 831.5 & 0.8505 \\
\hline 6 & & 831 & 0.8515 \\
\hline 7 & \multirow{3}{*}{392} & 852.5 & 1.0788 \\
\hline 8 & & 881.5 & 1.0090 \\
\hline 9 & & 877 & 1.0193 \\
\hline 10 & \multirow{3}{*}{490} & 918 & 1.1629 \\
\hline 11 & & 921.5 & 1.1541 \\
\hline 12 & & 916.5 & 1.1667 \\
\hline 13 & \multirow{3}{*}{720} & 956.5 & 1.5740 \\
\hline 14 & & 949 & 1.5989 \\
\hline 15 & & 959.5 & 1.5641 \\
\hline
\end{tabular}


Table 4-4. Calculated various parameters and fracture toughness for the basic indentation pressure approach

\begin{tabular}{|c|c|c|c|c|}
\hline $\begin{array}{c}\text { Average } \\
\text { indentation } \\
\text { diagonal } 2 l(\mu \mathrm{m})\end{array}$ & $n$ & $\begin{array}{l}\text { Microcrack } \\
\text { density } \rho_{c} \\
\left(1 / \mathrm{m}^{2}\right)\end{array}$ & $\begin{array}{l}\text { Strain energy } \\
\text { release rate } G_{S S} \\
\left(\mathrm{~J} / \mathrm{m}^{2}\right) \text { at } 25^{\circ} \mathrm{C}\end{array}$ & $\begin{array}{l}\text { Fracture toughness } \\
K_{S S}\left(\mathrm{MPa} \cdot \mathrm{m}^{1 / 2}\right) \\
\text { at } 25^{\circ} \mathrm{C}\end{array}$ \\
\hline 616.6 & 92.22 & $5.9596 \mathrm{e}+008$ & 13.4395 & 2.1397 \\
\hline 737.5 & 81.00 & $8.5257 \mathrm{e}+008$ & 13.4395 & 2.1397 \\
\hline 628.5 & 90.87 & $6.1918 \mathrm{e}+008$ & 13.4371 & 2.1395 \\
\hline 813 & 80.64 & $6.9071 \mathrm{e}+008$ & 13.4368 & 2.1395 \\
\hline 831.5 & 79.43 & $7.2250 \mathrm{e}+008$ & 13.4363 & 2.1394 \\
\hline 831 & 79.47 & $7.2163 \mathrm{e}+008$ & 13.4405 & 2.1398 \\
\hline 852.5 & 83.31 & $5.6959 \mathrm{e}+008$ & 13.4404 & 2.1398 \\
\hline 881.5 & 81.40 & $6.0901 \mathrm{e}+008$ & 13.4372 & 2.1395 \\
\hline 877 & 81.69 & $6.0280 \mathrm{e}+008$ & 13.4393 & 2.1397 \\
\hline 918 & 83.80 & $5.2839 \mathrm{e}+008$ & 13.4401 & 2.1397 \\
\hline 921.5 & 83.57 & $5.3242 \mathrm{e}+008$ & 13.4372 & 2.1395 \\
\hline 916.5 & 83.89 & $5.2666 \mathrm{e}+008$ & 13.4372 & 2.1395 \\
\hline 956.5 & 88.03 & $3.9039 \mathrm{e}+008$ & 13.4371 & 2.1395 \\
\hline 949 & 88.55 & $3.8429 \mathrm{e}+008$ & 13.4393 & 2.1397 \\
\hline 959.5 & 87.83 & $3.9284 \mathrm{e}+008$ & 13.4384 & 2.1396 \\
\hline
\end{tabular}


The calculated values of microcrack density, $\rho_{c}$, presented in Table 4-4 show that the microcrack density decreases with increasing indentation load due to the effect of substrate. The indenter goes deeper into the substrate with the applied indentation load increased. As a result, the substrate effect becomes more pronounced, the indentation pressure and the residual stress as well as the inter-crack spacing become larger, which causes the decrease of the calculated microcrack density. This trend is in agreement with the results obtained in the work of Nix and Gao [104]. In their model, as given by Eq. (3-10), the dislocation density was lower at larger indentation depth, and lower dislocation density might cause lower microcrack density as discussed previously, because microcracks were generated due to the dislocation movement of atoms.

The error of the experimental data should be analyzed before they are used for the calculations. To minimize the error, a group of diagonal length data is obtained on the indentation surface. As shown in Table 4-3, the average diagonal length (mean value of the two diagonal lengths) for each indentation impression ranges from $616.6 \mu \mathrm{m}$ to $737.5 \mu \mathrm{m}$ under the indentation load of $196 \mathrm{~N}$. The difference between the minimum and the maximum values reaches $120.9 \mu \mathrm{m}$ under $196 \mathrm{~N}$, while the maximum difference is $29 \mu \mathrm{m}$ under other indentation loads. Therefore, in order to select proper indentation diagonal length data for the fracture toughness calculation, the mean and maximum values of three indentation diagonal lengths under each applied load are given in Table 4-5. The graphs of indentation diagonal, 
$2 l$, indentation pressure, $p$, and microcrack density, $\rho_{c}$, versus indentation load are made in Figure 4-6.

Table 4-5. Calculated parameters based on mean and maximum indentation diagonal lengths for the basic indentation pressure approach

\begin{tabular}{|c|c|c|c|c|c|}
\hline & & $\begin{array}{l}\text { Indentation } \\
\text { diagonal } 2 l \\
(\mu \mathrm{m})\end{array}$ & Parameter $n$ & $\begin{array}{l}\text { Indentation } \\
\text { pressure } p \\
\qquad(\mathrm{GPa})\end{array}$ & $\begin{array}{c}\text { Microcrack } \\
\text { density } \rho_{c} \\
\left(1 / \mathrm{m}^{2}\right)\end{array}$ \\
\hline \multirow{2}{*}{$196 \mathrm{~N}$} & Mean & 660.9 & 87.52 & 0.8975 & $6.8467 \mathrm{e}+008$ \\
\hline & Max & 737.5 & 81.00 & 0.7207 & $8.5257 \mathrm{e}+008$ \\
\hline \multirow{2}{*}{$294 N$} & Mean & 825.2 & 79.84 & 0.8635 & $7.1160 \mathrm{e}+008$ \\
\hline & Max & 831.5 & 79.43 & 0.8505 & $7.2250 \mathrm{e}+008$ \\
\hline \multirow{2}{*}{$392 \mathrm{~N}$} & Mean & 870.3 & 82.12 & 1.0351 & $5.9363 e+008$ \\
\hline & Max & 881.5 & 81.40 & 1.0090 & $6.0901 \mathrm{e}+008$ \\
\hline \multirow{2}{*}{$490 \mathrm{~N}$} & Mean & 918.7 & 83.75 & 1.1611 & $5.2919 \mathrm{e}+008$ \\
\hline & Max & 921.5 & 83.57 & 1.1541 & $5.3242 \mathrm{e}+008$ \\
\hline \multirow{2}{*}{$720 \mathrm{~N}$} & Mean & 955 & 88.14 & 1.5789 & $3.8917 \mathrm{e}+008$ \\
\hline & Max & 959.5 & 87.83 & 1.5641 & $3.9284 \mathrm{e}+008$ \\
\hline
\end{tabular}




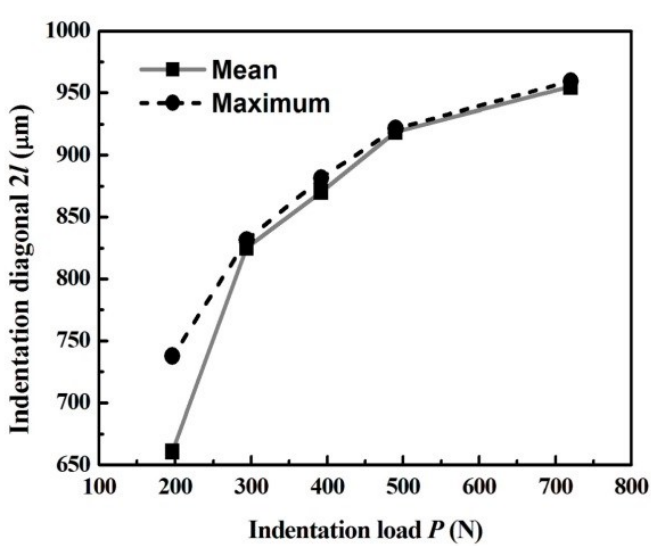

(a)

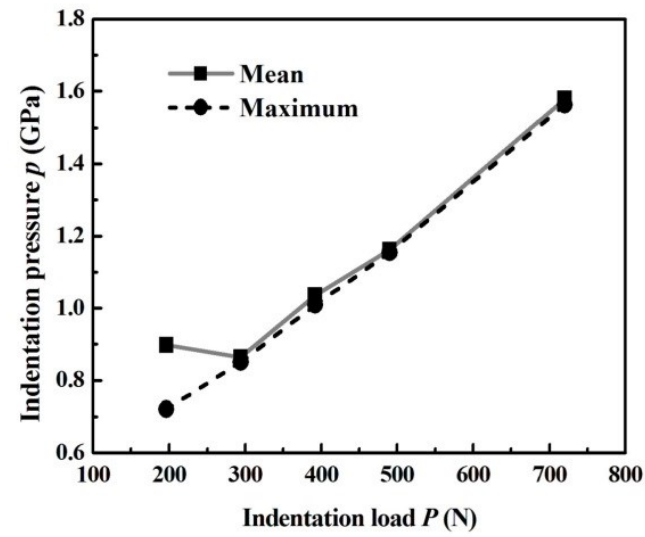

(c)

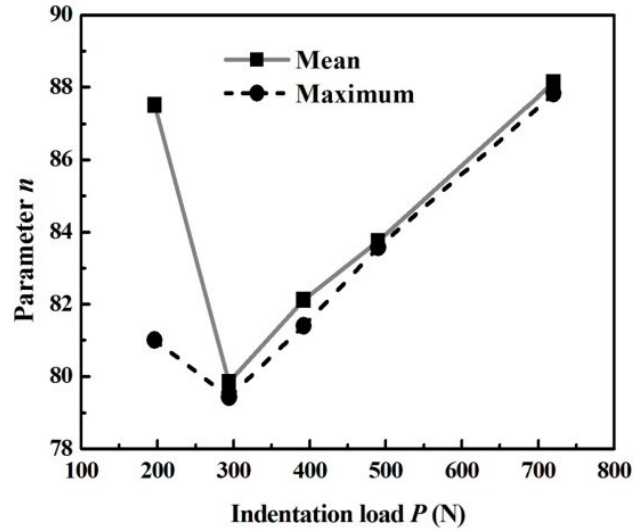

(b)

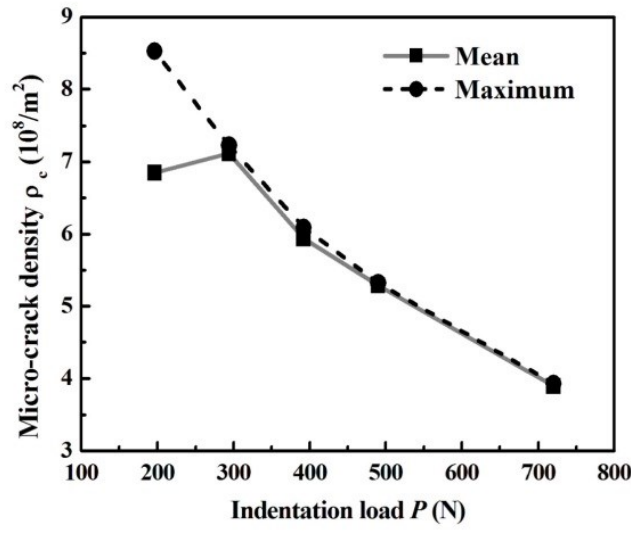

(d)

Figure 4-6. Indentation diagonal $2 l$ Parameters versus indentation load $P$ for the basic indentation pressure approach: (a) indentation diagonal $2 l$, (b) parameter $n$, (c) indentation pressure $p,(\mathrm{~d})$ microcrack density $\rho_{c}$. 
It is seen that the curves of indentation pressure and microcrack density corresponding to the maximum indentation diagonal lengths exhibit better continuity, while those for the mean indentation diagonal lengths have a sharp change at $294 \mathrm{~N}$. The difference in the values of parameter $n$ between $196 \mathrm{~N}$ and $294 \mathrm{~N}$ for the maximum diagonal lengths is also much smaller than that for the mean diagonal lengths. Generally, the mean diagonal lengths are applied to fracture toughness calculation, however, the maximum indentation diagonal lengths exhibit better continuity which is more reasonable. In order to investigate the fracture toughness variation with the applied load, both the maximum and mean diagonal lengths are used to investigate the temperature-dependent fracture toughness of the composite coating/substrate system. The calculated strain energy release rate, $G_{S S}$, and the fracture toughness, $K_{S S}$, of the composite coating/substrate system are mainly determined by indentation pressure, microcrack density and the parameter $n$. The fracture toughness values calculated at $298 \mathrm{~K}, 400 \mathrm{~K}, 600 \mathrm{~K}, 800 \mathrm{~K}$ and $1000 \mathrm{~K}$ are summarized in Table 4-6. 
Table 4-6. Calculated fracture toughness, $K_{S S}\left(\mathrm{MPa} \cdot \mathrm{m}^{1 / 2}\right)$, based on the basic indentation pressure approach

\begin{tabular}{|c|c|c|c|c|c|c|}
\hline \multicolumn{2}{|c|}{} & $298 \mathrm{~K}$ & $400 \mathrm{~K}$ & $600 \mathrm{~K}$ & $800 \mathrm{~K}$ & $1000 \mathrm{~K}$ \\
\hline \multirow{2}{*}{$196 \mathrm{~N}$} & Mean & 2.1396 & 3.5083 & 6.0260 & 8.2402 & 10.1978 \\
\cline { 2 - 7 } & Max & 2.1397 & 3.6078 & 6.3680 & 8.8273 & 11.0135 \\
\hline \multirow{2}{*}{$294 \mathrm{~N}$} & Mean & 2.1397 & 3.6275 & 6.4368 & 8.9464 & 11.1796 \\
\cline { 2 - 7 } & Max & 2.1394 & 3.6343 & 6.4612 & 8.9889 & 11.2399 \\
\hline \multirow{2}{*}{$392 \mathrm{~N}$} & Mean & 2.1396 & 3.5893 & 6.3038 & 8.7166 & 10.8585 \\
\cline { 2 - 7 } & Max & 2.1395 & 3.6009 & 6.3443 & 8.7866 & 10.9566 \\
\hline \multirow{2}{*}{$490 \mathrm{~N}$} & Mean & 2.1396 & 3.5634 & 6.2146 & 8.5632 & 10.6458 \\
\cline { 2 - 7 } & Max & 2.1395 & 3.5661 & 6.2241 & 8.5794 & 10.6688 \\
\hline
\end{tabular}

The fracture toughness of the composite coating/substrate system versus temperature for different indentation loads are plotted in Figure 4-7. These results are obtained from MATLAB calculations and the details are given in the Appendix. 


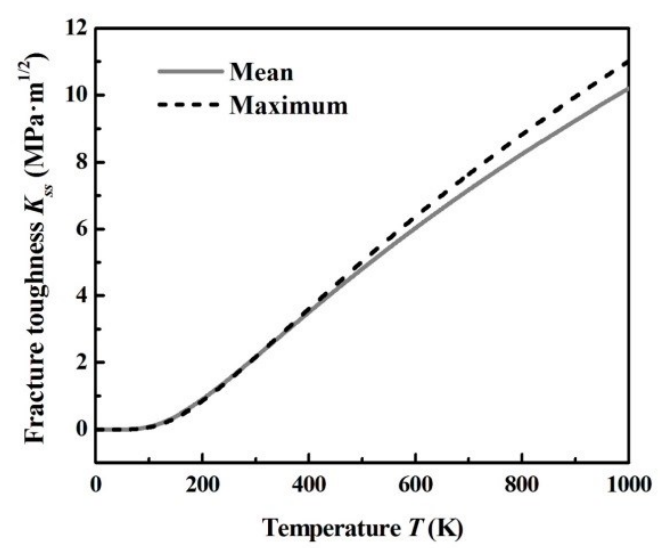

(a)

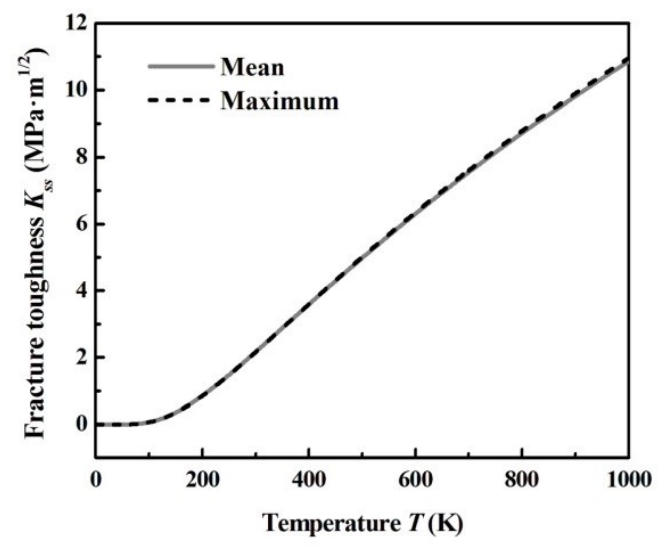

(c)

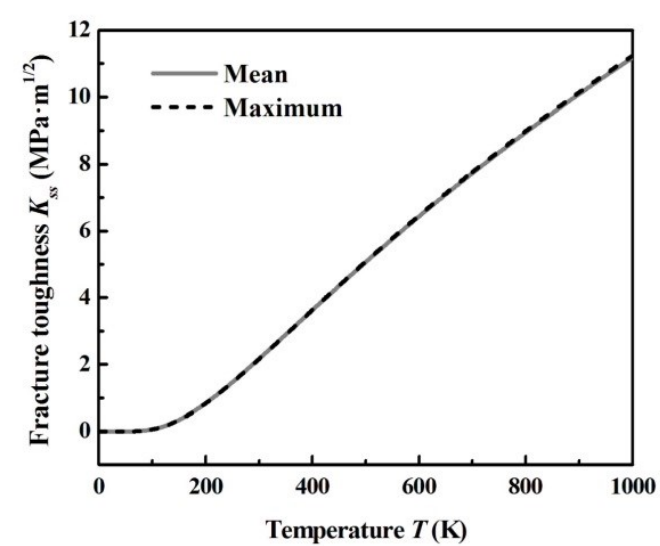

(b)

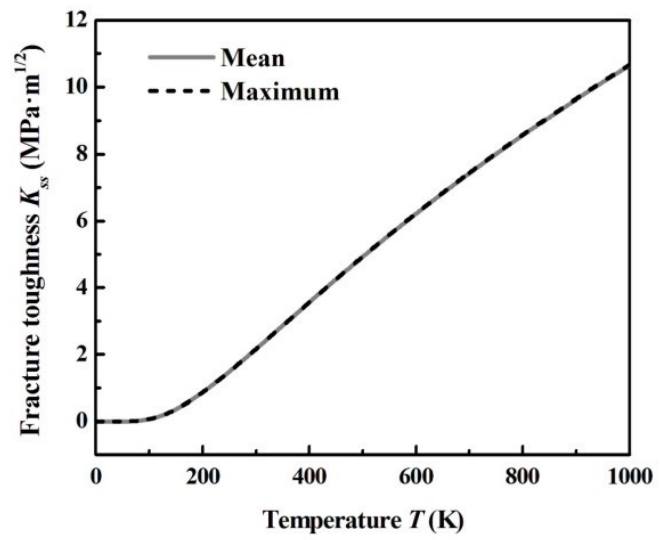

(d)

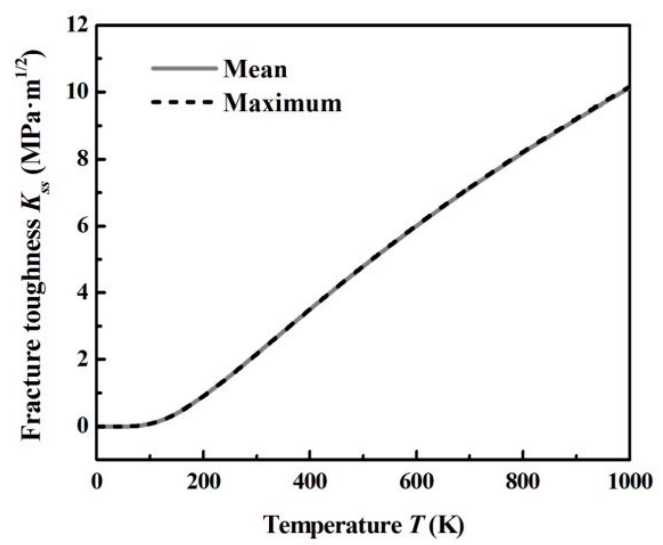

(e)

Figure 4-7. Temperature-dependent fracture toughness of the composite coating/substrate system under different indentation loads using the basic indentation pressure approach: (a) $196 \mathrm{~N}$, (b) $294 \mathrm{~N}$, (c) $392 \mathrm{~N}$, (d) $490 \mathrm{~N}$, (e) $720 \mathrm{~N}$. 
As shown in Figure 4-7, for each indentation load, the temperature-dependent fracture toughness remains unchanged at low temperatures, and increases rapidly from about $150 \mathrm{~K}$. The difference in the fracture toughness between using the maximum and mean indentation diagonal lengths is larger under $196 \mathrm{~N}$ due to the deviation of diagonal length data, and this difference is much smaller for other loads.

\subsubsection{Composite hardness approach}

As discussed previously, the substrate effect on the fracture toughness of coating/substrate systems can be included through incorporating the composite hardness given by Eq. (4-19). The calculated composite hardness for the composite coating/substrate system in terms of indentation pressure $p$ for different indentation impression diagonal lengths under each indentation load are given in Table 4-7. It is seen that the indentation pressure is inversely related to indentation load, because the substrate effect on the composite hardness is higher at larger indentation depth, and this trend is consistent with the indentation pressure test results of a brittle coating/substrate system given in Malzbender's work [136]. Based on the calculated composite hardness, the microcrack density, $\rho_{c}$, the parameter $n$, the strain energy release, $G_{S S}$, and the fracture toughness, $K_{S S}$, at room temperature for each indentation diagonal length are summarized in Table 4-8. 
Table 4-7. Average indentation diagonal $2 l$ and indentation pressure $p$ under each indentation load for the composite hardness approach

\begin{tabular}{|c|c|c|c|}
\hline $\begin{array}{c}\text { Indentation } \\
\text { mark \# }\end{array}$ & Load $P(\mathrm{~N})$ & $\begin{array}{l}\text { Average indentation } \\
\text { diagonal } 2 l(\mu \mathrm{m})\end{array}$ & $\begin{array}{c}\text { Indentation pressure } p \\
(\mathrm{GPa})\end{array}$ \\
\hline 1 & \multirow{3}{*}{196} & 616.6 & 1.5572 \\
\hline 2 & & 737.5 & 1.4740 \\
\hline 3 & & 628.5 & 1.5476 \\
\hline 4 & \multirow{3}{*}{294} & 813 & 1.4347 \\
\hline 5 & & 831.5 & 1.4261 \\
\hline 6 & & 831 & 1.4263 \\
\hline 7 & \multirow{3}{*}{392} & 852.5 & 1.4168 \\
\hline 8 & & 881.5 & 1.4048 \\
\hline 9 & & 877 & 1.4066 \\
\hline 10 & \multirow{3}{*}{490} & 918 & 1.3907 \\
\hline 11 & & 921.5 & 1.3894 \\
\hline 12 & & 916.5 & 1.3912 \\
\hline 13 & \multirow{3}{*}{720} & 956.5 & 1.3770 \\
\hline 14 & & 949 & 1.3795 \\
\hline 15 & & 959.5 & 1.3759 \\
\hline
\end{tabular}


Table 4-8. Calculated various parameters and fracture toughness for the composite hardness approach

\begin{tabular}{|c|c|c|c|c|}
\hline $\begin{array}{c}\text { Average } \\
\text { indentation } \\
\text { diagonal } 2 l(\mu \mathrm{m})\end{array}$ & $n$ & $\begin{array}{l}\text { Microcrack } \\
\text { density } \rho_{c} \\
\left(1 / \mathrm{m}^{2}\right)\end{array}$ & $\begin{array}{l}\text { Strain energy } \\
\text { release rate } G_{S S} \\
\left(\mathrm{~J} / \mathrm{m}^{2}\right) \text { at } 25^{\circ} \mathrm{C}\end{array}$ & $\begin{array}{c}\text { Fracture toughness } \\
K_{\text {sS }}\left(\mathrm{MPa} \cdot \mathrm{m}^{1 / 2}\right) \\
\text { at } 25^{\circ} \mathrm{C}\end{array}$ \\
\hline 616.6 & 109.72 & $3.9459 \mathrm{e}+008$ & 13.4395 & 2.1397 \\
\hline 737.5 & 107.01 & $4.1686 \mathrm{e}+008$ & 13.4380 & 2.1396 \\
\hline 628.5 & 109.41 & $3.9704 \mathrm{e}+008$ & 13.4399 & 2.1397 \\
\hline 813 & 96.20 & $4.2828 \mathrm{e}+008$ & 13.4401 & 2.1397 \\
\hline 831.5 & 95.96 & $4.3087 \mathrm{e}+008$ & 13.4376 & 2.1395 \\
\hline 831 & 95.97 & $4.3081 \mathrm{e}+008$ & 13.4392 & 2.1397 \\
\hline 852.5 & 92.08 & $4.3369 \mathrm{e}+008$ & 13.4399 & 2.1397 \\
\hline 881.5 & 91.78 & $4.3740 \mathrm{e}+008$ & 13.4405 & 2.1398 \\
\hline 877 & 91.82 & $4.3684 \mathrm{e}+008$ & 13.4385 & 2.1396 \\
\hline 918 & 89.42 & $4.4183 \mathrm{e}+008$ & 13.4388 & 2.1396 \\
\hline 921.5 & 89.39 & $4.4225 \mathrm{e}+008$ & 13.4394 & 2.1397 \\
\hline 916.5 & 89.43 & $4.4168 \mathrm{e}+008$ & 13.4380 & 2.1396 \\
\hline 956.5 & 83.89 & $4.4623 \mathrm{e}+008$ & 13.4376 & 2.1395 \\
\hline 949 & 83.95 & $4.4542 \mathrm{e}+008$ & 13.4405 & 2.1398 \\
\hline 959.5 & 83.87 & $4.4659 \mathrm{e}+008$ & 13.4393 & 2.1397 \\
\hline
\end{tabular}


As shown in Table 4-8, based on the composite hardness approach, the microcrack density, $\rho_{c}$, increase slightly with indentation load. With the increase of indentation load, the composite hardness, residual stresses and the inter-crack spacing in the plastic zone, all decrease, and the microcrack density is positively related to indentation load. However, the microcrack density is independent of the parameter $n$.

To ensure that the estimated strain energy release rate, $G_{S S}$, in Eq. (4-12) is the same as the strain energy release rate, $G_{e}$, at room temperature, the parameter $n$ varies with indentation load. Using the same indentation test data, the fracture toughness calculated from the Lawn's model given by Eq. (4-11) increased with indentation load [104]. To remove the load effect, a crack shape influence factor which is based on the ratio of crack length to crack depth was adopted in the fracture toughness calculation by several researchers [35,104,137]. In Demidova's work [18], the influence of indentation load was successfully offset by the crack shape influence factor, and the calculated fracture toughness is independent of indentation load in spite of the experimental error. The significance of the parameter $n$ in the present research is similar to that of the crack shape influence factor.

Similar to the basic indentation pressure approach, mean and maximum indentation diagonal lengths under each indentation load are selected for the fracture toughness calculation of the composite coating/substrate system and these data are summarized in Table 4-9. The graphs 
of indentation diagonal, $2 l$, indentation pressure, $p$, and microcrack density, $\rho_{c}$ versus indentation load are made in Figure 4-8.

Table 4-9. Calculated parameters based on mean and maximum indentation diagonal lengths for the composite hardness approach

\begin{tabular}{|c|c|c|c|c|c|}
\hline & & $\begin{array}{c}\text { Indentation } \\
\text { diagonal } 2 l \\
(\mu \mathrm{m})\end{array}$ & Parameter $n$ & $\begin{array}{l}\text { Indentation } \\
\text { pressure } p \\
\text { (GPa) }\end{array}$ & $\begin{array}{l}\text { Microcrack } \\
\text { density } \rho_{c} \\
\left(1 / \mathrm{m}^{2}\right)\end{array}$ \\
\hline \multirow{2}{*}{$196 \mathrm{~N}$} & Mean & 660.9 & 108.61 & 1.5232 & $4.0340 \mathrm{e}+008$ \\
\hline & Max & 737.5 & 107.01 & 1.4740 & $4.1686 \mathrm{e}+008$ \\
\hline \multirow{2}{*}{$294 N$} & Mean & 825.2 & 96.04 & 1.4290 & $4.2999 \mathrm{e}+008$ \\
\hline & Max & 831.5 & 95.96 & 1.4261 & $4.3087 \mathrm{e}+008$ \\
\hline \multirow{2}{*}{$392 \mathrm{~N}$} & Mean & 870.3 & 91.89 & 1.4093 & $4.3600 \mathrm{e}+008$ \\
\hline & Max & 881.5 & 91.78 & 1.4048 & $4.3740 \mathrm{e}+008$ \\
\hline \multirow{2}{*}{$490 \mathrm{~N}$} & Mean & 918.7 & 89.41 & 1.3904 & $4.4193 \mathrm{e}+008$ \\
\hline & Max & 921.5 & 89.39 & 1.3894 & $4.4225 \mathrm{e}+008$ \\
\hline \multirow{2}{*}{$720 \mathrm{~N}$} & Mean & 955 & 83.90 & 1.3775 & $4.4607 \mathrm{e}+008$ \\
\hline & Max & 959.5 & 83.87 & 1.3759 & $4.4659 \mathrm{e}+008$ \\
\hline
\end{tabular}




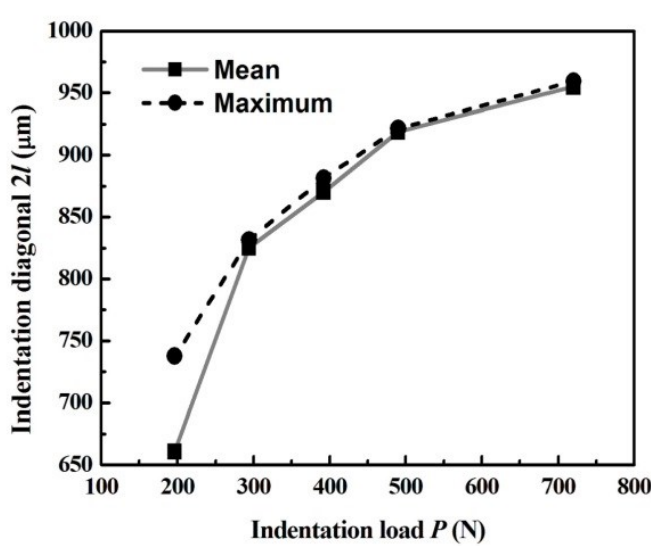

(a)

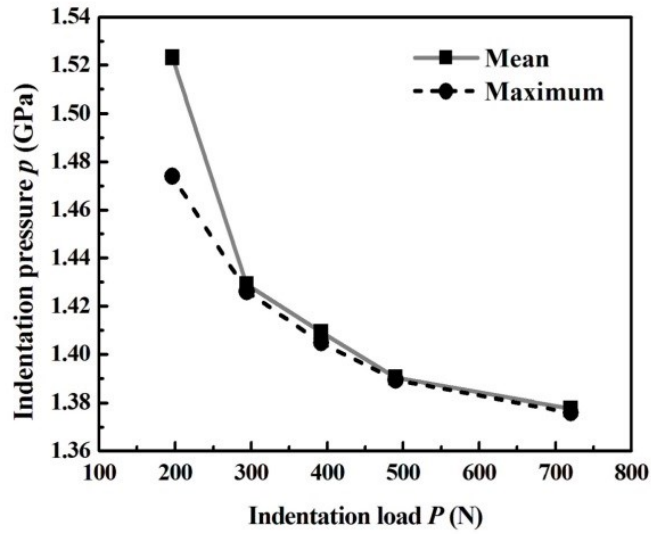

(c)

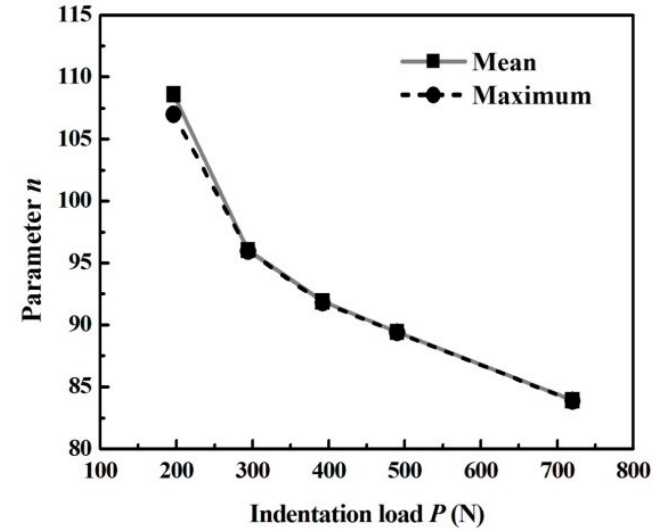

(b)

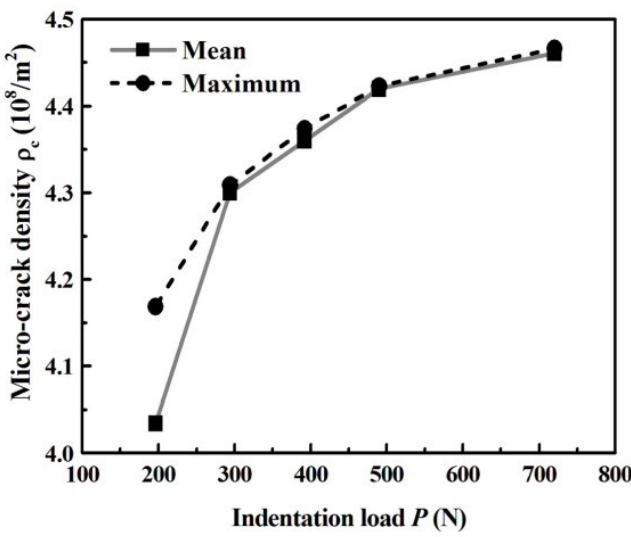

(d)

Figure 4-8. Indentation diagonal $2 l$ Parameters versus indentation load $P$ for the composite hardness approach: (a) indentation diagonal $2 l$, (b) parameter $n$, (c) indentation pressure $p$, (d) microcrack density $\rho_{c}$.

As discussed in last section, in order to eliminate the load effect on the fracture toughness, the parameter $n$ varies with indentation load and indentation diagonal length. Meanwhile, to evaluate the effect of the parameter $n$ on the fracture toughness, the value of this parameter is assumed unchanged to compare the room-temperature fracture toughness obtained under 
different indentation loads. Suppose that the value of $n$ is 107.01 which is obtained from the maximum indentation diagonal length under $196 \mathrm{~N}$. As shown in Table 4-10, with the same $n$ value, the calculated room-temperature fracture toughness ranges from $2.1396 \mathrm{MPa} \cdot \mathrm{m}^{1 / 2}$ to 2.9095 $\mathrm{MPa} \cdot \mathrm{m}^{1 / 2}$. Therefore, the parameter $n$, needs to be calculated separately for different indentation loads to minimize the load effect.

Table 4-10. Room-temperature fracture toughness based on the same parameter $n$

\begin{tabular}{|c|c|c|c|c|c|}
\hline & $196 \mathrm{~N}$ & $294 \mathrm{~N}$ & $392 \mathrm{~N}$ & $490 \mathrm{~N}$ & $720 \mathrm{~N}$ \\
\hline Fracture toughness $K_{S S}$ & 2.1396 & 2.4324 & 2.5742 & 2.6651 & 2.9095 \\
$\left(\mathrm{MPa} \cdot \mathrm{m}^{1 / 2}\right)$ at $25^{\circ} \mathrm{C}$ & & & & & \\
\hline
\end{tabular}

The calculated fracture toughness at $298 \mathrm{~K}, 400 \mathrm{~K}, 600 \mathrm{~K}, 800 \mathrm{~K}$ and $1000 \mathrm{~K}$ are reported in Table 4-11. The temperature-dependent fracture toughness for each indentation load is plotted in Figure 4-9. It is seen that the difference in the fracture toughness between using maximum and mean indentation diagonal lengths under $196 \mathrm{~N}$ is observed at high temperatures (above $600 \mathrm{~K}$ ), while the difference is hardly observed for other loads. However, the fracture toughness exhibits obvious increment with the applied load, which is caused by the large changes of parameter $n$ and indentation pressure, $p$. 
Table 4-11. Calculated fracture toughness, $K_{S S}\left(\mathrm{MPa} \cdot \mathrm{m}^{1 / 2}\right)$, using the composite hardness approach

\begin{tabular}{|c|c|c|c|c|c|c|}
\hline \multicolumn{2}{|c|}{} & $298 \mathrm{~K}$ & $400 \mathrm{~K}$ & $600 \mathrm{~K}$ & $800 \mathrm{~K}$ & $1000 \mathrm{~K}$ \\
\hline \multirow{2}{*}{$196 \mathrm{~N}$} & Mean & 2.1396 & 3.2794 & 5.2747 & 6.9800 & 8.4687 \\
\cline { 2 - 7 } & Max & 2.1396 & 3.2931 & 5.3185 & 7.0523 & 8.5669 \\
\hline \multirow{2}{*}{$294 \mathrm{~N}$} & Mean & 2.1396 & 3.4017 & 5.6703 & 7.6384 & 9.3673 \\
\cline { 2 - 7 } & Max & 2.1395 & 3.4026 & 5.6731 & 7.6432 & 9.3740 \\
\hline \multirow{2}{*}{$392 \mathrm{~N}$} & Mean & 2.1397 & 3.4509 & 5.8328 & 7.9122 & 9.7437 \\
\cline { 2 - 8 } & Max & 2.1398 & 3.4524 & 5.8376 & 7.9203 & 9.7548 \\
\hline \multirow{2}{*}{$490 \mathrm{~N}$} & Mean & 2.1396 & 3.4825 & 5.9391 & 8.0924 & 9.9924 \\
\cline { 2 - 7 } & Max & 2.1397 & 3.4830 & 5.9403 & 8.0944 & 9.9951 \\
\hline
\end{tabular}




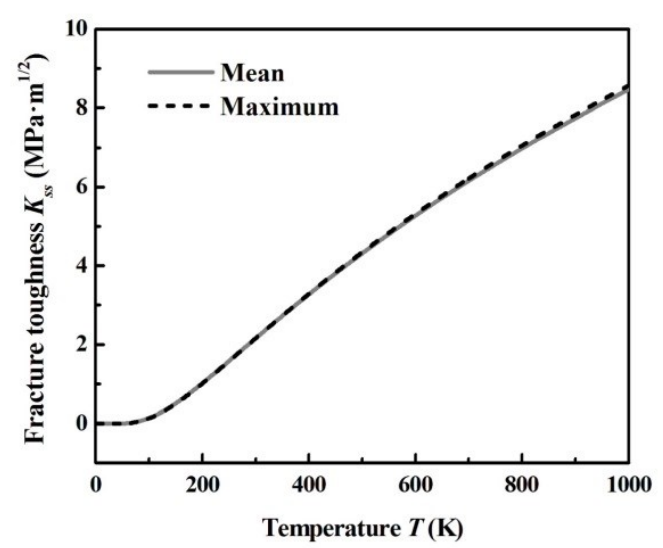

(a)

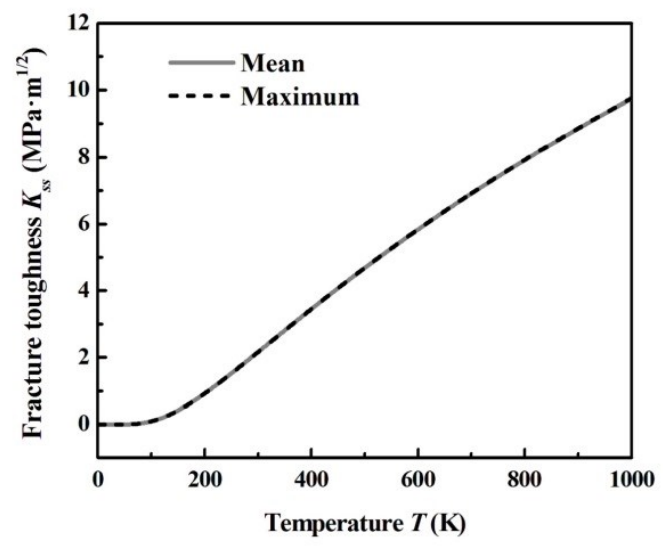

(c)

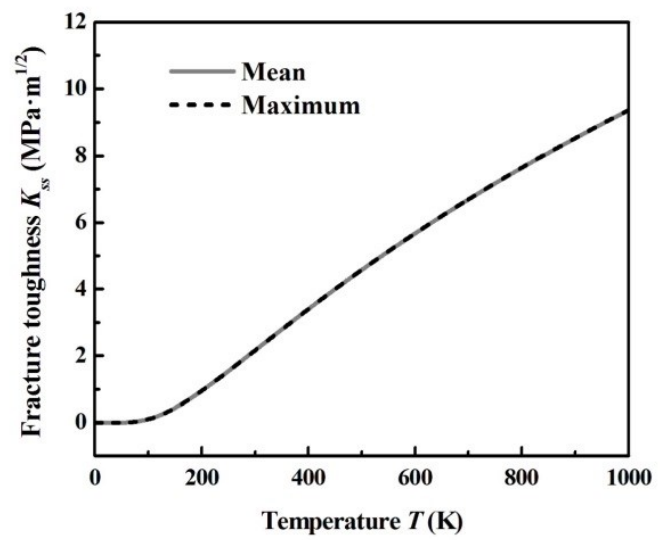

(b)

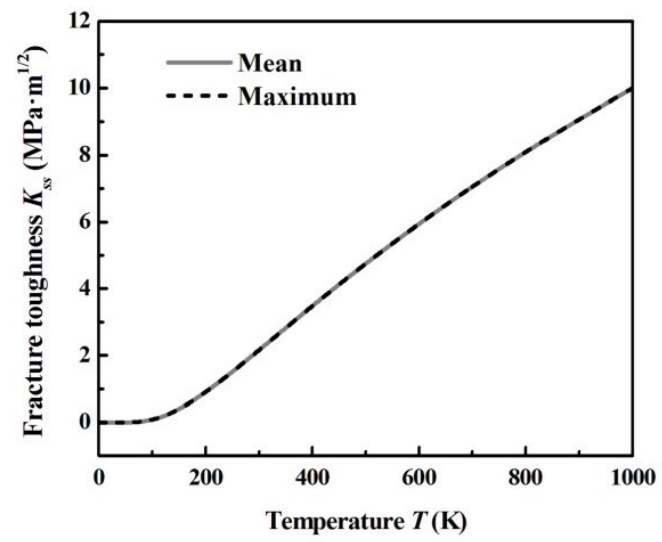

(d)

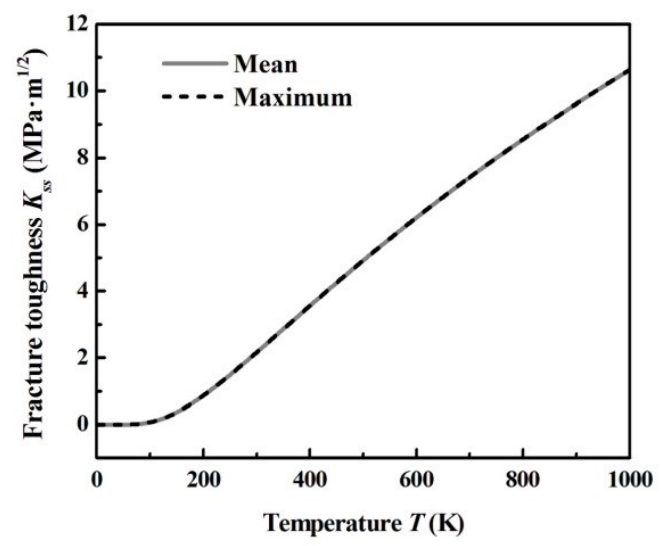

(e)

Figure 4-9. Temperature-dependent fracture toughness under different indentation loads using the composite hardness approach: (a) $196 \mathrm{~N}$, (b) $294 \mathrm{~N}$, (c) $392 \mathrm{~N}$, (d) $490 \mathrm{~N}$, (e) $720 \mathrm{~N}$. 


\subsection{Closing Remarks}

In this chapter, microcrack-formation-based fracture toughness models for $\mathrm{WC}-10 \mathrm{Co} 4 \mathrm{Cr}$ coating/1018 low carbon steel substrate system at high temperatures are proposed. By using the basic indentation pressure approach and the composite hardness approach, respectively, two models are developed. Arrhenius-type equation and the fracture toughness model developed in Hu's work [9] are applied. The calculations of microcrack density and microcrack area are included in the model to evaluate the load effect on the fracture toughness of $\mathrm{WC}-10 \mathrm{Co} 4 \mathrm{Cr}$ coating/1018 low carbon steel system. Two parameters, $m$ and $n$, are introduced in these models. The parameter $m$ relates the total growth of microcrack in terms of crack tip opening displacement to the magnitude of dislocation movement of atoms according to Burgers vector. The scale-linking parameter $n$ which relates the micro-level energy barrier to the strain energy release rate is used to minimize the load effect at room temperature, which is similar to the function of crack shape influence factor.

The data of the radial crack length and the indentation impression diagonal are adopted in these models, which were obtained by Demidova [18]. They are analyzed in this chapter to minimize the effect of the experimental data error on the fracture toughness evaluation. The calculation results show that, in addition to the maximum indentation diagonal lengths, the parameter $n$, the indentation pressure as well as the microcrack density that are obtained using the maximum indentation diagonal data all exhibit better continuity with the indentation load, which indicates that using the maximum indentation diagonal lengths for 
the fracture toughness calculations are more reasonable. Therefore, the calculated fracture toughness results that will be compared in Chapter 5 are all obtained using the maximum indentation diagonal data. 


\section{Chapter 5. Discussion of Results}

The fracture toughness and parameters derived from the two approaches in Chapter 4 are compared, and the comparison of the fracture toughness obtained from the models developed in this research is implemented in this chapter.

\subsection{Comparison of Results between Two Approaches}

According to the results derived from the two approaches in Chapter 4, the variations of indentation pressure, $p$, parameter $n$, microcrack density, $\rho_{c}$, and strain energy release, $G_{S S}$, with indentation load are very different. These parameters exhibit better continuity when the maximum rather than the mean indentation diagonal lengths are used in the calculations due to data deviation. Therefore, in order to minimize the effect of data error on the fracture toughness comparison, all of the results compared in this chapter are obtained based on the maximum indentation diagonal lengths under each load. For simplicity, in the following comparison analyses, the basic indentation pressure approach is named approach 1 and the composite hardness approach is named approach 2.

As illustrated in Figure 5-1, the indentation pressure calculated from both approaches are very close to the substrate hardness, indicating that the substrate effect might have been taken in the calculation of the indentation pressure. However, the variation range of indentation 
pressure from approach 2 is much smaller than that from approach 1. The comparisons of microcrack density and parameter $n$, between the two approaches are illustrated in Figure 5-2.

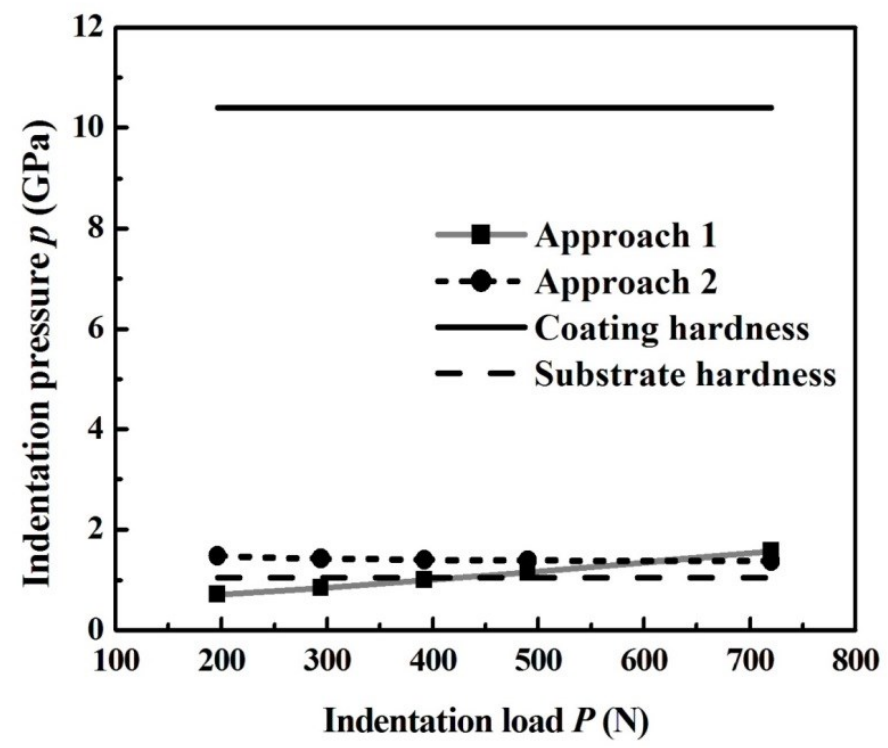

Figure 5-1. Indentation pressure $p$ versus applied load $P$.

According to Figure 5-2(a), the microcrack density calculated using approach 1 is more sensitive to indentation load than approach 2 . This is caused by the variation of residual stress with indentation pressure, as described by Eq. (4-17). However, the microcrack density variation with indentation load calculated from approach 1 is consistent with the calculation results of Nix and Gao [104]. In their calculation using Eq. (3-10), the dislocation density was lower at larger indentation depth, and lower dislocation density might cause lower microcrack density, as microcracks result from the movement of dislocations. 


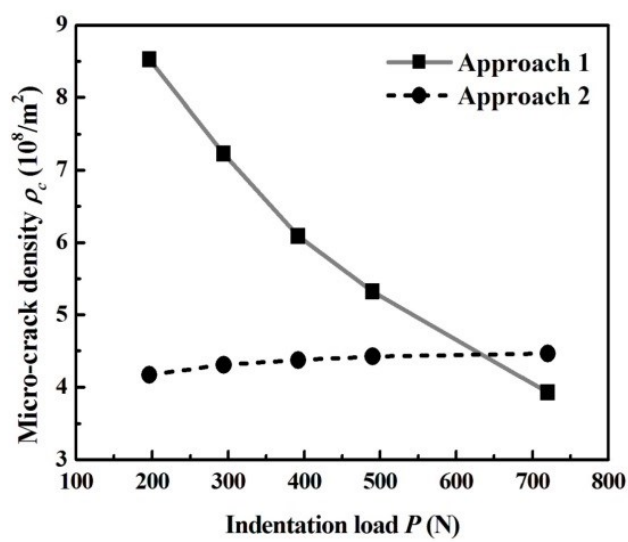

(a)

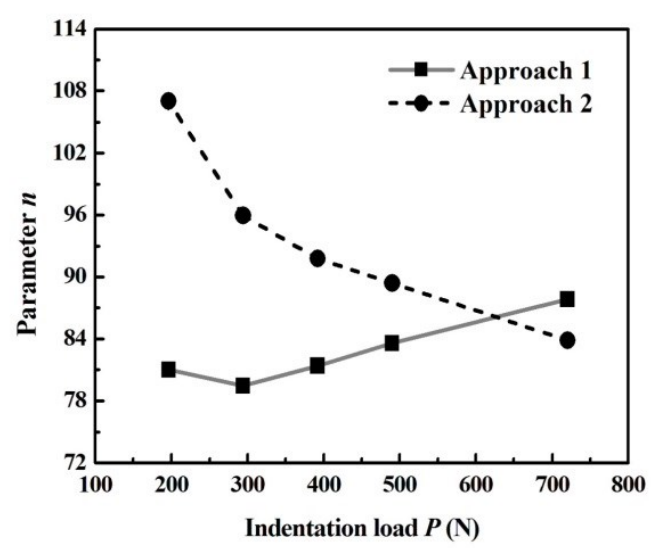

(b)

Figure 5-2. Parameters versus indentation load $P$ : (a) microcrack density $\rho_{c}$, (b) parameter $n$.

The parameter $n$ from approach 1 varies less than that from approach 2 according to the curves in Figure 5-2(b). This means that the load effect on the room-temperature fracture toughness is much less when the basic indentation pressure estimation method is used in the calculation. The parameter $n$ is introduced to minimize the effect of indentation load on the calculated room-temperature fracture toughness and strain energy release rate, however, the calculation results are still subject to load effect at high temperatures. Therefore, based on Figure 5-2(b), the fracture toughness and strain energy release rate estimated from approach 1 are more stable with the increasing applied load at high temperatures as shown in Figure 5-3, and the comparison between calculated fracture toughness and experimental data $[18,119,120]$ is given in Figure 5-4. 


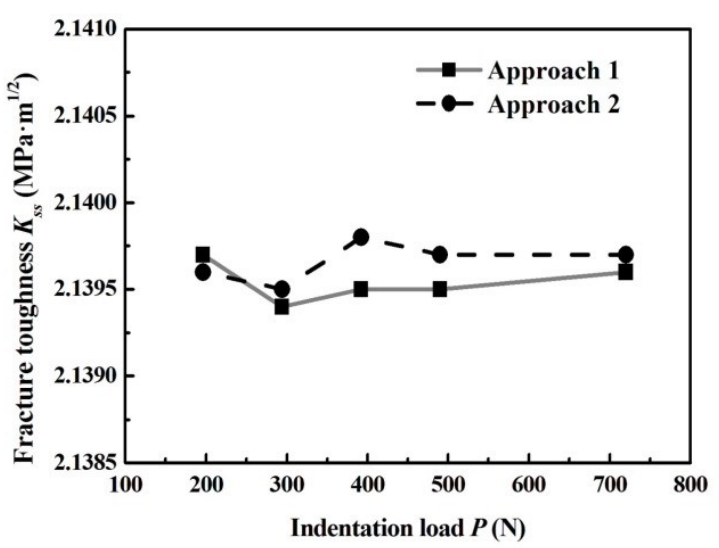

(a)

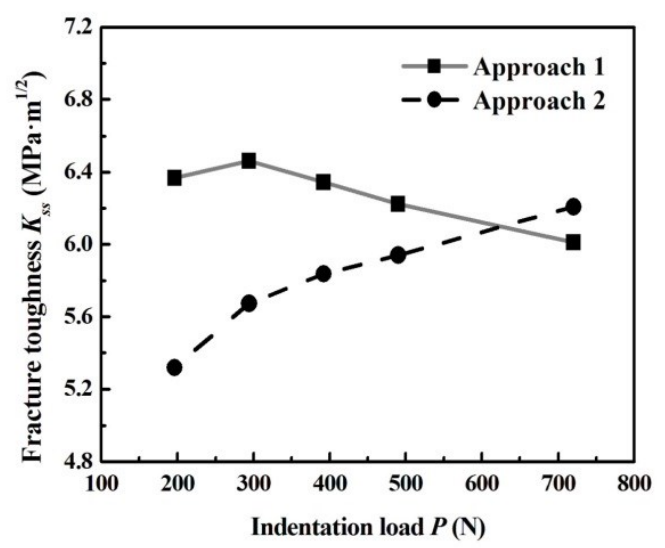

(c)

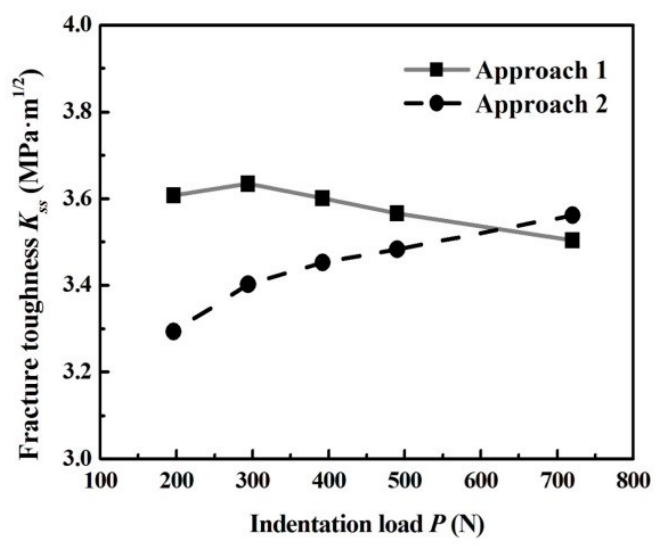

(b)

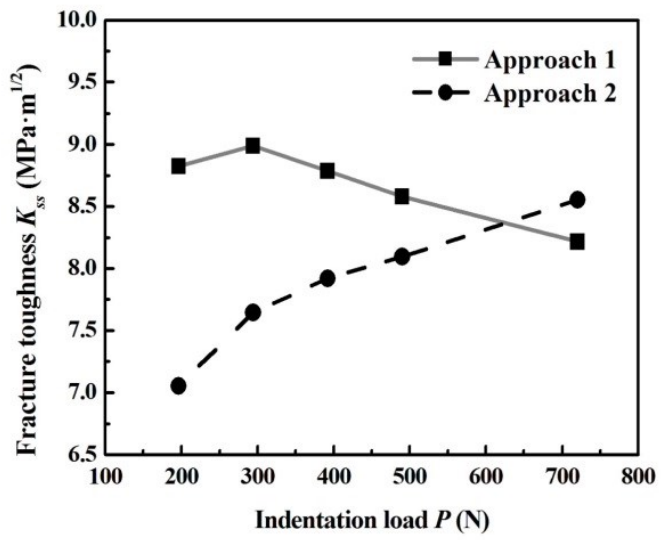

(d)

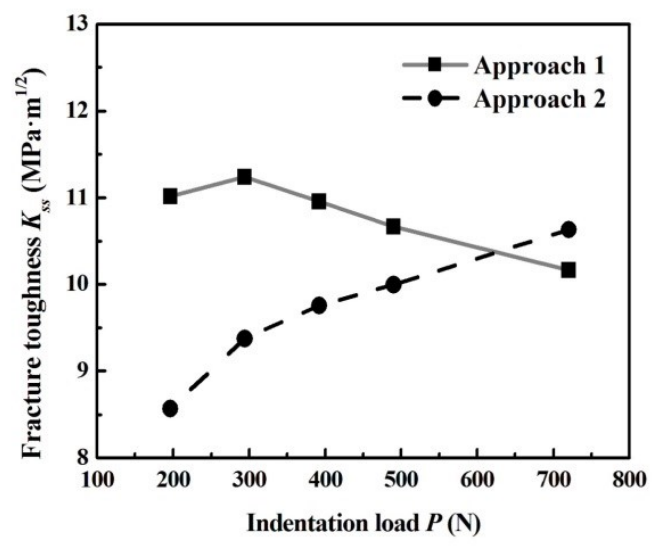

(e)

Figure 5-3. Comparison of calculated fracture toughness for the composite coating/substrate system between two approaches: (a) $298 \mathrm{~K}$, (b) $400 \mathrm{~K}$, (c) $600 \mathrm{~K}$, (d) $800 \mathrm{~K}$, (e) $1000 \mathrm{~K}$. 
It is seen that, at $400 \mathrm{~K}$, the fracture toughness for approach 1 ranges from $3.5039 \mathrm{MPa} \cdot \mathrm{m}^{1 / 2}$ to $3.6343 \mathrm{MPa} \cdot \mathrm{m}^{1 / 2}$, and the fracture toughness calculated using approach 2 varies from 3.2931 $\mathrm{MPa} \cdot \mathrm{m}^{1 / 2}$ to $3.5618 \mathrm{MPa} \cdot \mathrm{m}^{1 / 2}$. The fracture toughness variation range of approach 2 is larger than that of approach 1, and this trend become more obvious at high temperatures. At $1000 \mathrm{~K}$, the results from approach 1 increase from $10.1624 \mathrm{MPa} \cdot \mathrm{m}^{1 / 2}$ to 11.2399 $\mathrm{MPa} \cdot \mathrm{m}^{1 / 2}$ with the increase of indentation load, the variation range is about $1 \mathrm{MPa} \cdot \mathrm{m}^{1 / 2}$. While the difference of the minimum and maximum fracture toughness calculated from approach 2 is about $2 \mathrm{MPa} \cdot \mathrm{m}^{1 / 2}$. Therefore, the load effect on the fracture toughness calculated from approach 1 is smaller.

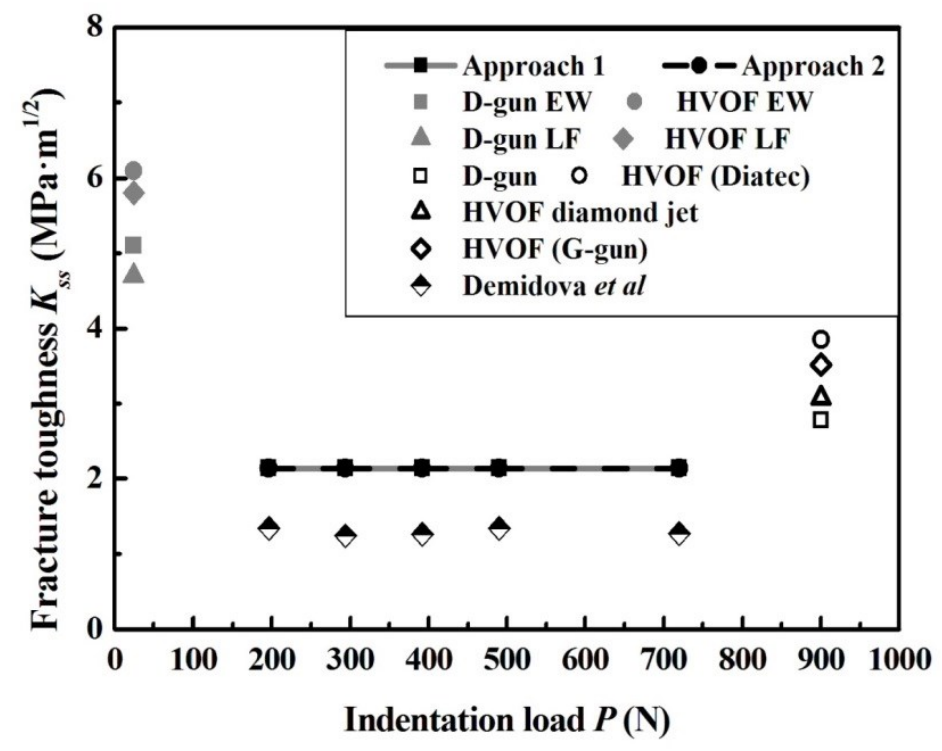

Figure 5-4. Comparison between calculated fracture toughness and experimental data at room temperature $[18,119,120]$.

As shown in Figure 5-4 at room temperature $(298 \mathrm{~K})$, the fracture toughness values for the composite coating/substrate system calculated from the two approaches and the experimental data obtained by other researchers have the same order of magnitudes $[18,119,120]$. It is seen 
that, for the same composite coating, due to the different process methods, calculation models, and other factors such as grain size, the experimental results varies from $1.2 \mathrm{MPa} \cdot \mathrm{m}^{1 / 2}$ to 6.1 $\mathrm{MPa} \cdot \mathrm{m}^{1 / 2}$. Therefore, the fracture toughness obtained from the two approaches is in a reasonable range.

\subsection{Comparison of Results from Different Models}

As reported in previous chapters, two models that incorporate individual phase crystal structures in the fracture toughness calculation are developed by modifying an existing model in Chapter 3 and two models that are based on microcrack formation theory are proposed in Chapter 4. These models are all employed to evaluate the fracture toughness of $\mathrm{WC}-10 \mathrm{Co} 4 \mathrm{Cr}$ coating/1018 low carbon steel substrate system at high temperatures. Further comparison is made between these models. Depending on the influence factors considered in the crystal-structure-based models, the mixture methods used for integrating the fracture toughness of individual phases include the basic mixture method and the unconstrained mixture method. The latter has been validated by previous experimental data [105], while the former estimates the integrated fracture toughness only based on the volume fraction of each phase, which does not conform to the real condition. Therefore, only the crystal-structure-based model using the unconstrained mixture method participates in the comparison of this chapter, named modified model for simplicity. For the microcrack-formation-based models in Chapter 4, to facilitate the comparison, the model created using the basic indentation pressure approach (approach 1) is named proposed model 
1 and that developed with the composite hardness approach (approach 2) is named proposed model 2.

In order to compare the high-temperature fracture toughness results from different models, the parameter $n$ was calculated separately for different loads and different models to make sure that the calculated room-temperature fracture toughness obtained from the three models are almost the same. The maximum and minimum fracture toughness results obtained from proposed model 1 and proposed model 2 are shown in Figure 5-5. It is seen that when the temperature is increased, the rising trends of the three curves are very similar. At $1000 \mathrm{~K}$, the fracture toughness obtained from the modified model in Chapter 3 is $10.815 \mathrm{MPa} \cdot \mathrm{m}^{1 / 2}$, and the results using the proposed model 1 in Chapter 4 ranges from $10.1624 \mathrm{MPa} \cdot \mathrm{m}^{1 / 2}$ to 11.2399 $\mathrm{MPa} \cdot \mathrm{m}^{1 / 2}$, which is in good agreement with the results obtained from the modified model. The fracture toughness evaluated using the proposed model 2 in Chapter 4 exhibits lower increase rate with temperature than that using the other two models at low indentation loads, but at high loads, the three models behave similarly. In addition, it is interesting to notice that the fracture toughness values obtained from the modified model and the proposed model 1 do not change much with indentation load, but that derived from the proposed model 2 increase from $8.5669 \mathrm{MPa} \cdot \mathrm{m}^{1 / 2}$ to $10.6308 \mathrm{MPa} \cdot \mathrm{m}^{1 / 2}$ with the load increase from $196 \mathrm{~N}$ to $720 \mathrm{~N}$. This is because the parameter $n$ has a large variation range for the proposed model 2 in order to ensure that the calculated room-temperature fracture toughness and strain 
energy release rate from Eq. (4-12) are consistent with the results obtained from Hu's model (Eq. (4-10) and Eq. (4-11)).

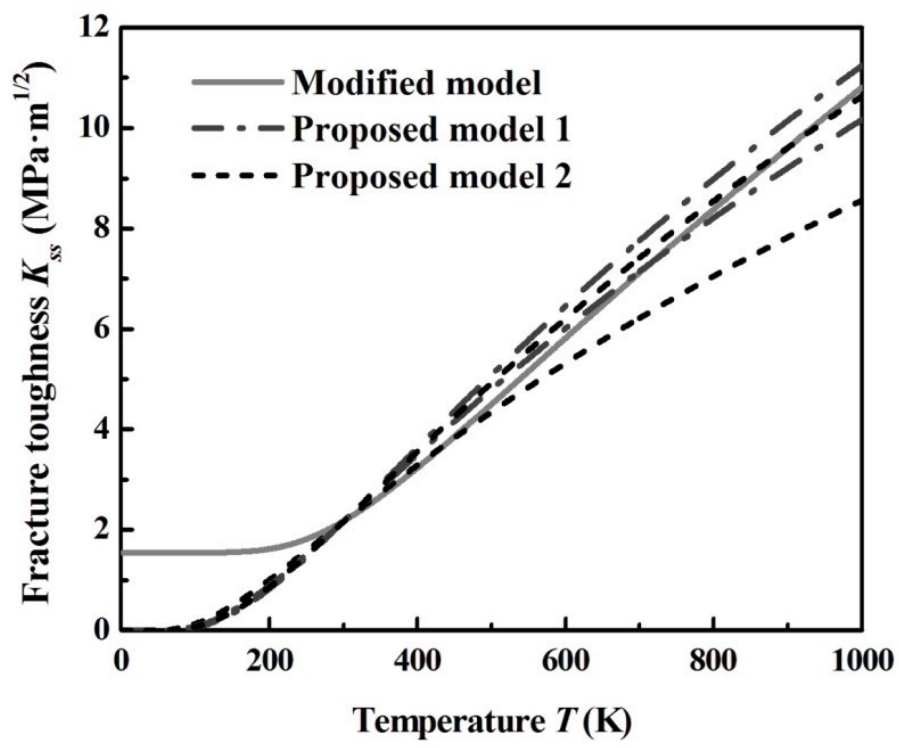

Figure 5-5. Maximum and minimum temperature-dependent fracture toughness obtained from three models.

At the low temperatures (below $298 \mathrm{~K}$ ), the fracture toughness calculated from the modified model has a minimum of $1.54145 \mathrm{MPa} \cdot \mathrm{m}^{1 / 2}$ and increases to $2.1567 \mathrm{MPa} \cdot \mathrm{m}^{1 / 2}$ at room temperature. However, the fracture toughness calculated using the proposed models are almost equal to zero at low temperatures. In the modified model, the strain energy release rate is divided into two parts: the work done by elastic and plastic deformations. The elastic deformation is estimated from the surface energy of the composite coating which is independent of temperature, while the plastic deformation is expressed by a temperature related function, as described by Eq. (3-4b). This means that when the work done by plastic 
deformation is much smaller than that done by elastic deformation, the fracture behavior of the system would be governed by elastic component.

In the proposed models, both the plastic and elastic deformations are included in the strain energy release rate equation, as expressed by Eq. (4-10b). To predict the fracture toughness of the composite coating/substrate system precisely at low temperatures, a surface energy related initial value should be given, which means that the fracture toughness model should be a piecewise function. However, the initial value that is based on surface energy and Young's modulus needs to be determined through low temperature experiment which is inaccessible in the present research.

The parameter $n$ used in the proposed models needs to be determined by using the room-temperature indentation diagonal length data, which were obtained from indentation tests. As shown in Figure 5-5, based on the calculated parameter $n$, the high-temperature fracture toughness of the composite coating/substrate system predicted via the two proposed models are in a reasonable range when compared with the results obtained from the modified model. Therefore, the temperature-dependent fracture toughness models developed in Chapter 4 are applicable to the fracture toughness evaluation for brittle coating/ductile substrate systems at high temperatures (above $298 \mathrm{~K}$ ). 


\section{Chapter 6. Conclusions and Future Work}

\subsection{Summary}

In this research, two types of temperature-dependent fracture toughness models were developed, which are applied on WC-10Co4Cr coating/1018 low carbon steel system. The crystal structure type is obtained via modifying an existing model that is based on the Arrhenius-type equation and rate controlling theory, where the model parameters are fitted to the crack type and geometries generated in the composite coating/substrate system under indentation loading. The two main phases of WC-10Co4Cr composite are analyzed based on its chemical composition; they are brittle $\delta$-WC phase and ductile $\alpha$-Co phase. The microstructures and the slip systems of these phases are studied and discussed based on the phase diagrams and literature, it is shown that the Burgers vector of $1 / 3[1 \overline{2} 10]$ on prismatic slip plane $\{10 \overline{1} 0\}$ is responsible for the slip system of $\delta$-WC phase, and the Burgers vector of $1 / 2[110]$ on the slip plane $\{111\}$ is responsible for the slip system of $\alpha$-Co phase.

The crystal-structure-based temperature-dependent fracture toughness model developed at microscopic level considers the radial crack propagation in the brittle coating/ductile substrate system under indentation loading. The behavior of radial cracks generated in the coating and fully developed during the unloading process is applied to the fracture toughness estimation of the composite coating/substrate system, and the residual stresses in terms of the energy stored in the plastic zone during the loading process are the main driving forces for the propagation of the radial cracks. 
The fracture toughness of $\mathrm{WC}-10 \mathrm{Co} 4 \mathrm{Cr}$ coating/1018 low carbon steel substrate system is determined by integrating the fracture toughness of $\delta$-WC and $\alpha$-Co using the basic mixture method and the unconstrained mixture method, respectively. The former shows deficiency and the latter is proven applicable when compared with the experimental data from other studies. The fracture toughness of the composite coating/substrate system remains unchanged until the temperature reaches a critical value. Then the fracture toughness increases rapidly in an exponential manner. The critical temperatures for $\delta$-WC and $\alpha$-Co coatings are $600 \mathrm{~K}$ and $200 \mathrm{~K}$, respectively. This is mainly determined by the relationship between the work done by elastic and plastic deformations. The estimated fracture toughness for the composite coating/substrate system has an increment from $2.1567 \mathrm{MPa} \cdot \mathrm{m}^{1 / 2}$ to $10.815 \mathrm{MPa} \cdot \mathrm{m}^{1 / 2}$ over a temperature range from $298 \mathrm{~K}$ to $1000 \mathrm{~K}$. The room-temperature fracture toughness is in the reasonable range when compared with the results of the same coating derived in other researches. The fracture toughness estimated from the crystal-structure-based model using the unconstrained mixture method is independent of indentation load, which is consistent with the definition of fracture toughness as a material property.

The microcrack formation type includes the models derived from the basic indentation pressure approach and the composite hardness approach, respectively. Although both models take the substrate effect into consideration, the variations of indentation pressure, $p$, parameter $n$, microcrack density, $\rho_{c}$, and strain energy release, $G_{s S}$, with indentation load are very different. As the temperature is increased, the rising trends of the fracture toughness 
obtained from the two models are very similar. At $1000 \mathrm{~K}$, the fracture toughness obtained from the basic indentation pressure approach ranges from $10.1624 \mathrm{MPa} \cdot \mathrm{m}^{1 / 2}$ to 11.2399 $\mathrm{MPa} \cdot \mathrm{m}^{1 / 2}$, which is in good agreement with the results obtained from the modified model in Chapter 3. The fracture toughness evaluated from the model using the composite hardness approach exhibits lower increase rate with temperature than that from the model using the basic indentation pressure approach at low indentation loads, but at high loads, the two models behave similarly. In addition, the fracture toughness obtained from the basic indentation pressure approach is more stable with indentation load when compared with that derived from the composite hardness approach, which increases from $8.5669 \mathrm{MPa} \cdot \mathrm{m}^{1 / 2}$ to $10.6308 \mathrm{MPa} \cdot \mathrm{m}^{1 / 2}$ with the load increase from $196 \mathrm{~N}$ to $720 \mathrm{~N}$. This is because the parameter $n$ has a large variation range for the model using the composite hardness approach.

\subsection{Conclusions}

The main objective of this research, developing effective models for evaluating the fracture toughness of brittle coating/ductile substrate systems at high temperatures, has been achieved. Based on the summary addressed above, the following conclusions can be drawn from this research:

1. The two main phases that constitute the $\mathrm{HVOF}$ WC- $10 \mathrm{Co} 4 \mathrm{Cr}$ composite coating are brittle $\delta$-WC phase and ductile $\alpha$-Co phase. Based on the microstructures and the slip 
systems, the Burgers vector of $1 / 3[1 \overline{2} 10]$ on prismatic slip plane $\{10 \overline{1} 0\}$ is responsible for the slip system of $\delta$-WC phase, and the Burgers vector of $1 / 2[110]$ on the slip plane $\{111\}$ is responsible for the slip system of $\alpha$-Co phase.

2. The dislocation density of individual phases in a coating can be estimated analytically based on its crystal structure. The geometries of plastic zone and radial cracks are taken into account in the fracture toughness model based on crystal structure analysis.

3. The radial cracks generated in the coating and fully developed during the unloading process of indentation can be applied to the fracture toughness estimation of brittle coating/ductile substrate systems, and the residual stresses due to the energy stored in plastic zone during the loading process are the main driving forces for the propagation of the radial cracks.

4. For the crystal-structure-based models, the fracture toughness of the composite coating is obtained by integrating the fracture toughness of $\delta$-WC and the $\alpha$-Co phases. The model using the unconstrained mixture method has been validated by the existing experimental data, but the one from the basic mixture method has obvious drawbacks in the formulation. The fracture toughness estimated using the crystal-structure-based models is independent of indentation load, which is consistent to the nature of material properties. 
5. The fracture toughness of $\mathrm{WC}-10 \mathrm{Co} 4 \mathrm{Cr}$ coating/1018 low carbon steel system remains unchanged until the temperature reaches a certain value. It then increases rapidly in an exponential manner. The fracture toughness of brittle coating is lower than that of bulk material with similar constitution. The relationship between the work done by elastic and plastic deformations determines the critical temperature of fracture toughness increase. The critical temperatures for $\delta-\mathrm{WC}$ and $\alpha$-Co coatings are $600 \mathrm{~K}$ and $200 \mathrm{~K}$, respectively. From room-temperature to $1000 \mathrm{~K}$, the calculated fracture toughness increases from $2.1567 \mathrm{MPa} \cdot \mathrm{m}^{1 / 2}$ to $10.815 \mathrm{MPa} \cdot \mathrm{m}^{1 / 2}$ in an exponential manner.

6. The crack tip opening displacement (CTOD) can be used to measure the total growth of a microcrack in tensile direction, and the dislocation movement can be associated with the crack propagation in order to estimate the fracture toughness of coating/substrate systems.

7. For the microcrack-formation-based models, both the models using the basic indentation pressure approach and using the composite hardness approach take the substrate effect into the fracture toughness estimation. The fracture toughness variation against indentation load is influenced by the calculated indentation pressure. 
8. The fracture toughness of $\mathrm{WC}-10 \mathrm{Co} 4 \mathrm{Cr}$ coating/1018 low carbon steel system estimated using the microcrack-formation-based models is influenced by indentation load at high temperatures, but the variation is within a reasonable range.

9. The microcrack-formation-based models are applicable to assess the fracture toughness of the composite coating/substrate system at high temperatures (above $298 \mathrm{~K}$ ). However, a surface energy and Young's modulus related initial value needs to be known in order to predict the fracture toughness of the system precisely at low temperatures, which should be obtained experimentally.

10. At room temperature, the fracture toughness values obtained from the crystal-structure-based and microcrack-formation-based models are consistent. With the increase in temperature, the ascending trends of the values are very similar. The fracture toughness evaluated from the microcrack-formation-based model using the composite hardness approach shows lower increase rate with temperature than that from the other models under low indentation loads, but in the high-load condition, all models behave similarly. The room-temperature fracture toughness obtained from all models is about 2.1 $\mathrm{MPa} \cdot \mathrm{m}^{1 / 2}$, the calculated fracture toughness at $1000 \mathrm{~K}$ ranges from $8.5669 \mathrm{MPa} \cdot$ $\mathrm{m}^{1 / 2}$ to $11.2399 \mathrm{MPa} \cdot \mathrm{m}^{1 / 2}$ with different models and indentation loads. 


\subsection{Main Contributions}

Although there are many valuable outcomes derived from this research, the main contributions can be summarized as follows:

Effective analytical models for evaluating the fracture toughness of brittle coating/ductile substrate systems at high temperatures have been devised. These models can predict the high-temperature fracture toughness of brittle coating/ductile substrate systems using room-temperature indentation test data, which significantly solves the problem that high-temperature indentation test equipment is not always available. For the crystal-structure-based models, as long as the microstructure and associated phases of the coating are known, the fracture toughness of each phase in the coating can be determined analytically, according to the crystal structures and lattice parameters of the phases. Then using the unconstrained mixture method, the overall fracture toughness of the coating can be evaluated by integrating the fracture toughness of individual phases. The crystal-structure-based models calculate the fracture toughness of coatings independent of indentation load, which is consistent with the nature of material properties. On the other hand, for the microcrack-formation-based models, using the crack profiles determined in indentation tests at room temperature, along with the properties of coating and substrate materials such as Young's modulus and yield strength, the fracture toughness of brittle coating/ductile substrate systems at high temperatures can be calculated, and all the models 
take the substrate influence into the fracture toughness estimation of the coating/substrate system.

\subsection{Future Work}

Based on the temperature-dependent fracture toughness models developed in this research, the calculated fracture toughness of $\mathrm{WC}-10 \mathrm{Co} 4 \mathrm{Cr}$ coating/1018 low carbon steel substrate system is $2.1567 \mathrm{MPa} \cdot \mathrm{m}^{1 / 2}$ at room temperature, and ranges from $8.5669 \mathrm{MPa} \cdot \mathrm{m}^{1 / 2}$ to 11.2399 $\mathrm{MPa} \cdot \mathrm{m}^{1 / 2}$ using different models. It has been shown that the results calculated from the microcrack-formation-based models are consistent with that obtained from the crystal-structure-based model (unconstrained mixture method).

However, some critical parameters included in these models such as lattice constant, Young's modulus, yield stress and surface energy may change at elevated temperatures. These parameters are obtained from literature, and their variations with the temperature are not taken into consideration due to their inaccessibility. Relevant high-temperature experiments need to be implemented to examine these parameters.

To further improve and verify these models, the indentation tests under high loads need to be conducted at high temperatures, in order to obtain the crack profiles in such conditions. The models proposed in this research can be further improved by completely removing the load effect from the fracture toughness calculations. Due to the change of materials at high 
temperatures, for example, softening, the crack profiles in coating/substrate systems generated under indentation at room temperature will be different from those at high temperatures. Attempts have been made to obtain such crack profiles in this research, but unfortunately, it became impossible either because of the insufficient load capacity of a high-temperature micro-hardness tester or limited temperature capacity of a macro-hardness test machine. Further attempt has been planned to find a hot indentation tester with high load capacity, so that the developed models can be improved. 


\section{References}

[1] G. Qian, T. Nakamura, C. C. Berndt, and S. H. Leigh, 'Tensile Toughness Test and High Temperature Fracture Analysis of Thermal Barrier Coatings', Acta Materialia, 45 (1997), 1767-84.

[2] M. Jafari, M. H. Enayati, M. Salehi, S. M. Nahvi, and C. G. Park, 'Comparison between Oxidation Kinetics of HVOF Sprayed WC-12Co and WC-10Co-4Cr Coatings', International Journal of Refractory Metals and Hard Materials, 41 (2013), 78-84.

[3] E. S. Puchi-Cabrera, M. H. Staia, Y. Y. Santana, E. J. Mora-Zorrilla, J. Lesage, D. Chicot, J. G. La Barbera-Sosa, E. Ochoa-Perez, and C. J. Villalobos-Gutierrez, 'Fatigue Behavior of Aa7075-T6 Aluminum Alloy Coated with a WC-10Co-4Cr Cermet by HVOF Thermal Spray', Surface and Coatings Technology, 220 (2013), 122-30.

[4] M. Yandouzi, and B. Jodoin, 'Cermet Coatings Prepared by Pulsed Gas Dynamic Spraying Process: Effect of the Process Parameters', Surface and Coatings Technology, 203 (2008), 104-14.

[5] H. J. C. Voorwald, R. C. Souza, W. L. Pigatin, and M. O. H. Cioffi, 'Evaluation of WC17Co and WC-10Co-4Cr Thermal Spray Coatings by HVOF on the Fatigue and Corrosion Strength of AISI 4340 Steel', Surface and Coatings Technology, 190 (2005), 155-64.

[6] M. Yandouzi, L. Ajdelsztajn, and B. Jodoin, 'WC-Based Composite Coatings Prepared by the Pulsed Gas Dynamic Spraying Process: Effect of the Feedstock Powders', Surface and Coatings Technology, 202 (2008), 3866-77.

[7] D. Mari, B. Clausen, M. A. M. Bourke, and K. Buss, 'Measurement of Residual Thermal Stress in WC-Co by Neutron Diffraction', International Journal of Refractory Metals and Hard Materials, 27 (2009), 282-87.

[8] A. Ghabchi, T. Varis, E. Turunen, T. Suhonen, X. Liu, and S. P. Hannula, 'Behavior of HVOF WC-10Co4Cr Coatings with Different Carbide Size in Fine and Coarse Particle Abrasion', Journal of Thermal Spray Technology, 19 (2009), 368-77.

[9] M. S. Hu, and A. G. Evans, 'The Cracking and Decohesion of Thin Films on Ductile Substrates', Acta Metallurgica, 37 (1989), 917-25.

[10] B. A. Latella, B. K. Gan, K. E. Davies, D. R. McKenzie, and D. G. McCulloch, 'Titanium Nitride/Vanadium Nitride Alloy Coatings: Mechanical Properties and Adhesion Characteristics', Surface and Coatings Technology, 200 (2006), 3605-11. 
[11] G. T. Hahn, M. F. Kanninen, and A. R. Rosenfield, 'Fracture Toughness of Materials', Annual Review of Materials Science, 2 (1972), 381-404.

[12] S. Zhang, D. Sun, Y. Fu, and H. Du, 'Toughness Measurement of Thin Films: A Critical Review', Surface and Coatings Technology, 198 (2005), 74-84.

[13] G. R. Anstis, P. Chantikul, B. R. Lawn, and D. B. Marshall, 'A Critical Evaluation of Indentation Techniques for Measuring Fracture Toughness: I, Direct Crack Measurements', Journal of the American Ceramic Society, 64 (1981), 533-38.

[14] K. Chan, 'Relationships of Fracture Toughness and Dislocation Mobility in Intermetallics', Metallurgical and Materials Transactions A, 34 (2003), 2315-28.

[15] B. R. Lawn, A. G. Evans, and D. B. Marshall, 'Elastic/Plastic Indentation Damage in Ceramics: The Median/Radial Crack System', Journal of the American Ceramic Society, 63 (1980), 574-81.

[16] R. F. Cook, and G. M. Pharr, 'Direct Observation and Analysis of Indentation Cracking in Glasses and Ceramics', Journal of the American Ceramic Society, 73 (1990), 787-817.

[17] K. Durst, B. Backes, and M. Göken, 'Indentation Size Effect in Metallic Materials: Correcting for the Size of the Plastic Zone', Scripta Materialia, 52 (2005), 1093-97.

[18] N. V. Demidova, 'An Improved Approach to Fracture Toughness Assessment of Brittle Coating on Ductile Substrate Systems under Indentation', PhD thesis, Carleton Unicersity, (2010), pp. 87-89.

[19] M. Ortiz, 'Microcrack Coalescence and Macroscopic Crack Growth Initiation in Brittle Solids', International Journal of Solids and Structures, 24 (1988), 231-50.

[20] A. A. Griffith, 'The Phenomena of Rupture and Flow in Solids', Philosophical Transactions of the Royal Society of London. Series A, Containing Papers of a Mathematical or Physical Character, 221 (1921), 163-98.

[21] W. D. Callister, D. G. Rethwisch, Materials Science and Engineering-An Introduction, Wiley, (2013).

[22] G. R. Irwin, 'Analysis of Stresses and Strains Near the End of a Crack Traversing a Plate', J. Appl. Mech. 24 (1957) 361-364.

[23] C. Sun and Z. Jin, Fracture Mechanics, ISBN: 978-0-12-385001-0, Academic Press, October 14, 2011, pp. 60. 
[24] H. M. Westergaard, 'Bearing Pressures and Cracks', J. Appl. Mech. 6 (1939) 49-53.

[25] G. C. Sih, 'On the Westergaard Method of Crack Analysis', Int. J. Fract. Mech. 2 (1966) $628-631$.

[26] G. T. Hahn, M. F. Kanninen, and A. R. Rosenfield, 'Fracture Toughness of Materials', Annual Review of Materials Science, 2 (1972), 381-404.

[27] S. Zhang, and X. Zhang, 'Toughness Evaluation of Hard Coatings and Thin Films', Thin Solid Films, 520 (2012), 2375-89.

[28] A. G. Evans, and E. A. Charles, 'Fracture Toughness Determinations by Indentation', Journal of the American Ceramic Society, 59 (1976), 371-72.

[29] M. L. Emiliaini, 'Debond Coating Requirements for Brittle Matrix Composites', Journal of Materials Science, 28 (1993), 5280-96.

[30] P. Kodali, K. C. Walter, and M. Nastasi, 'Investigation of Mechanical and Tribological Properties of Amorphous Diamond-Like Carbon Coatings', Tribology International, 30, 591-98.

[31] H. Li, K. A. Khor, and P. Cheang, 'Young's Modulus and Fracture Toughness Determination of High Velocity Oxy-Fuel-Sprayed Bioceramic Coatings', Surface and Coatings Technology, 155, 21-32.

[32] D. B. Marshall, and A. G. Evans, 'Reply to "Comment on 'Elastic/Plastic Indentation Damage in Ceramics: The Median/Radial Crack System'"', Journal of the American Ceramic Society, 64 (1981), C-182-C-83.

[33] D. B. Marshall, and B. R. Lawn, 'An Indentation Technique for Measuring Stresses in Tempered Glass Surfaces', Journal of the American Ceramic Society, 60 (1977), 86-87.

[34] J. Malzbender, and G. de With, 'Elastic Modulus, Hardness and Fracture Toughness of Sio2-Filled Methyltrimethoxysilane Coatings on Glass Substrates', Journal of Non-Crystalline Solids, 265 (2000), 51-60.

[35] J. Malzbender, J. M. J. den Toonder, A. R. Balkenende, and G. de With, 'Measuring Mechanical Properties of Coatings: A Methodology Applied to Nano-Particle-Filled Sol-Gel Coatings on Glass', Materials Science and Engineering: R: Reports, 36, 47-103.

[36] X. Li, D. Diao, and B. Bharat, 'Fracture Mechanisms of Thin Amorphous Carbon Films in Nanoindentation', Acta Materialia, 45, 4453-61. 
[37] X. Li and B. Bharat, 'Measurement of Fracture Toughness of Ultra-Thin Amorphous Carbon Films', Thin Solid Films, 315, 214-21.

[38] J. D. Toonder, J. Malzbender, G. De With, and R. Balkenende, 'Fracture Toughness and Adhesion Energy of Sol-Gel Coatings on Glass', Journal of Materials Research, 17 (2002), 224-33.

[39] A. G. Evans, M. D. Drory, and M. S. Hu, 'The Cracking and Decohesion of Thin Films', Journal of Materials Research, 3 (1988), 1043-49.

[40] D. B. Marshall, B. N. Cox, and A. G. Evans, 'The Mechanics of Matrix Cracking in Brittle-Matrix Fiber Composites', Acta Metallurgica, 33 (1985), 2013-21.

[41] Y. Wang, A. Hamza, and E. Ma, 'Temperature-Dependent Strain Rate Sensitivity and Activation Volume of Nanocrystalline Ni', Acta Materialia, 54 (2006), 2715-26.

[42] E. Harry, A. Rouzaud, M. Ignat, and P. Juliet, 'Mechanical Properties of W and W(C) Thin Films: Young's Modulus, Fracture Toughness and Adhesion', Thin Solid Films, 332 (1998), 195-201.

[43] E. Harry, M. Ignat, Y. Pauleau, A. Rouzaud, and P. Juliet, 'Mechanical Behaviour of Hard PVD Multilayered Coatings', Surface and Coatings Technology, 125 (2000), 185-89.

[44] E. Harry, M. Ignat, A. Rouzaud, and P. Juliet, 'Cracking Investigation of W and W(C) Films Deposited by Physical Vapor Deposition on Steel Substrates', Surface and Coatings Technology, 111 (1999), 177-83.

[45] B. J. Dalgleish, K. P. Trumble, and A. G. Evans, 'The Strength and Fracture of Alumina Bonded with Aluminum Alloys', Acta Metallurgica, 37 (1989), 1923-31.

[46] J. S. Wang, Y. Sugimura, A. G. Evans, and W. K. Tredway, 'The Mechanical Performance of DLC Films on Steel Substrates', Thin Solid Films, 325 (1998), 163-74.

[47] B. A. Latella, B. K. Gan, K. E. Davies, D. R. McKenzie, and D. G. McCulloch, 'Titanium Nitride/Vanadium Nitride Alloy Coatings: Mechanical Properties and Adhesion Characteristics', Surface and Coatings Technology, 200 (2006), 3605-11.

[48] B. P. Somerday, Y. Leng, and R. P. Gangloff, 'Elevated Temperature Fracture of Particulate-Reinforced Aluminum Part II: Micromechanical Modelling', Fatigue \& Fracture of Engineering Materials \& Structures, 18 (1995), 1031-50. 
[49] M. J. Haynes, and R. P. Gangloff, 'Elevated Temperature Fracture Toughness of Al-Cu-Mg-Ag Sheet: Characterization and Modeling', Metallurgical and Materials Transactions A, 28 (1997), 1815-29.

[50] M. J. Haynes, B. P. Somerday, C. L. Lach, and R. P. Gangloff, 'Micromechanical Modeling of Temperature-Dependent Initiation Fracture Toughness in Advanced Aluminum Alloys', Elevated Temperature Effects on Fatigue and Fracture, ASTM STP 1297, R. S. Piascik, R. P. Gangloff, and A. Saxena, Ed., American Society for Testing and Materials, 1997, pp. 165-190.

[51] W. Grellmann, and M. Che, 'Assessment of Temperature-Dependent Fracture Behavior with Different Fracture Mechanics Concepts on Examples of Unoriented and Cold-Rolled Polypropylene', Journal of Applied Polymer Science, 66 (1997), 1237-49.

[52] D. M. Bahr and W. S. Johnson, 'Temperature Dependent Fracture Toughness of a Single Crystal Nickel Superalloy', Fatigue and Fracture Mechanics, 34 th Volume, ASTM STP 1461, Steven R. Daniewicz, James C. Newman, and Karl-Heinz Schwalbe, Ed., American Society for Testing and Materials, 2005, pp. 340-352.

[53] W. C. Porr, and R. P. Gangloff, 'Elevated Temperature Fracture of RS/PM Alloy 8009: Part I. Fracture Mechanics Behavior', Metallurgical and Materials Transactions A, 25 (1994), 365-79.

[54] A. A. Griffith, 'The Phenomena of Rupture and Flow in Solids', Philosophical Transactions of the Royal Society of London A: Mathematical, Physical and Engineering Sciences, 221 (1921), 163-98.

[55] C. E. Inglis, Stresses in a Plate due to the Presence of Cracks and Sharp Corners, Trans. Inst. Naval. Arch., London, 55, 219-230, 1913.

[56] A. S. Krausz, K. Krausz, Fracture Kinetics of Crack Growth, ISBN: 978-94-009-1381-3, Kluwer Academic Publishers, 1988, pp. 8.

[57] Q. Wei, S. Cheng, K. T. Ramesh, and E. Ma, 'Effect of Nanocrystalline and Ultrafine Grain Sizes on the Strain Rate Sensitivity and Activation Volume: fcc Versus bcc Metals', Materials Science and Engineering: A, 381 (2004), 71-79.

[58] A. S. Krausz, H. Eyring, 'Chemical Kinetics of Plastic Deformation', Journal of Applied Physics, 42 (1971), 2382. 
[59] A. S. Krausz, and K. Krausz, 'The Theory of Thermally Activated Fracture: Environment Assisted Crack Propagation', Canadian Metallurgical Quarterly, 23 (1984), 107-13.

[60] A. S. Krausz, K. Krausz, Unified Constitutive Laws of Plastic Deformation, ISBN: 978-0-12-425970-6, Academic Press, 1996, pp. 247.

[61] A. S. Krausz, and K. Krausz, 'The Deformation Kinetics Theory of the Stress Dependence of Plastic Deformation', Acta Metallurgica et Materialia, 42 (1994), 937-45.

[62] W. R. Tyson, and H. Conrad, 'Plastic Deformation of Hafnium-Oxygen Alloys', Metallurgical Transactions, 4 (1973), 2605-11.

[63] B. K. Atkinson, 'A Fracture Mechanics Study of Subcritical Tensile Cracking of Quartz in Wet Environments', pure and applied geophysics, 117 (1979), 1011-24.

[64] S. V. Raj, and S. C. Farmer, 'Characteristics of a New Creep Regime in Polycrystalline Nial', Metallurgical and Materials Transactions A, 26 (1995), 343-56.

[65] C. Yin, M. Döner, and H. Conrad, 'Deformation Kinetics of Commercial Ti-50a (0.5 At. Pct Oeq) at Low Temperatures (T<0.3t M )', Metallurgical Transactions A, 14 (1983), 2545-56.

[66] H. Conrad, 'Grain Size Dependence of the Plastic Deformation Kinetics in Cu', Materials Science and Engineering: A, 341 (2003), 216-28.

[67] N. Yazdipour, and P. D. Hodgson, 'Modelling Post-Deformation Softening Kinetics of 304 Austenitic Stainless Steel Using Cellular Automata', Computational Materials Science, 54 (2012), 56-65.

[68] Y. Wang, A. Hamza, and E. Ma, 'Temperature-Dependent Strain Rate Sensitivity and Activation Volume of Nanocrystalline Ni', Acta Materialia, 54 (2006), 2715-26.

[69] A. G. Atkins, C. S. Lee, and R. M. Caddell, 'Time-Temperature Dependent Fracture Toughness of Pmma', Journal of Materials Science, 10 (1975), 1381-404.

[70] D. C. Phillips, J. M. Scott, and M. Jones, 'Crack Propagation in an Amine-Cured Epoxide Resin', Journal of Materials Science, 13 (1978), 311-22.

[71] B. Z. Margolin, A. G. Gulenko, and V. A. Shvetsova, 'Improved Probabilistic Model for Fracture Toughness Prediction for Nuclear Pressure Vessel Steels', International Journal of Pressure Vessels and Piping, 75 (1998), 843-55. 
[72] T. Kawasaki, S. Nakanishi, Y. Sawaki, K. Hatanaka, and T. Yokobori, 'Fracture Toughness and Fatigue Crack Propagation in High Strength Steel from Room Temperature to $-180^{\circ} \mathrm{C}^{\prime}$, Engineering Fracture Mechanics, 7 (1975), 465-72.

[73] R. Huang, Z. Suo, J. H. Prevost, and W. D. Nix, 'Inhomogeneous Deformation in Metallic Glasses', Journal of the Mechanics and Physics of Solids, 50, 1011-27.

[74] P. Gumbsch, 'Brittle Fracture and the Brittle-to-Ductile Transition of Tungsten', Journal of Nuclear Materials, 323 (2003), 304-12.

[75] X. H. Zeng, and A. Hartmaier, 'Modeling Size Effects on Fracture Toughness by Dislocation Dynamics', Acta Materialia, 58 (2010), 301-10.

[76] J. J. Brennan, and K. M. Prewo, 'Silicon Carbide Fibre Reinforced Glass-Ceramic Matrix Composites Exhibiting High Strength and Toughness', Journal of Materials Science, 17 (1982), 2371-83.

[77] O. Sano, and Y. Kudo, 'Relation of Fracture Resistance to Fabric for Granitic Rocks', pure and applied geophysics, 138 (1992), 657-77.

[78] J. R. Rice, 'A Path Independent Integral and the Approximate Analysis of Strain Concentration by Notches and Cracks', Journal of Applied Mechanics, 35 (1968), 379-86.

[79] G. B. Gibbs, 'The Thermodynamics of Thermally-Activated Dislocation Glide', physica status solidi (b), 10 (1965), 507-12.

[80] K. Chan, and D. L Davidson, 'Effects of Ti Addition on Cleavage Fracture in Nb-Cr-Ti Solid-Solution Alloys', Metallurgical and Materials Transactions A, 30 (1999), 925-39.

[81] K. Chan, 'Alloying Effects on the Fracture Toughness of Nb-Based Silicides and Laves Phases', Materials Science and Engineering: A, 409 (2005), 257-69.

[82] A. S. Kurlov, and A. I. Gusev, 'Tungsten Carbides and W-C Phase Diagram', Inorganic Materials, 42 (2006), 121-27.

[83] A.S. Kurlov, and A.I. Gusev, Tungsten Carbides: Structure, Properties and Application in Hardmetals, Springer International Publishing, 2013, p19.

[84] L. M. Berger, P. Ettmayer, P. Vuoristo, T. Mäntylä, and W. Kunert, 'Microstructure and Properties of WC-10\%Co-4\%Cr Spray Powders and Coatings: Part 1. Powder Characterization', Journal of Thermal Spray Technology, 10, 311-25. 
[85] M. Yandouzi, L. Ajdelsztajn, and B. Jodoin, 'WC-Based Composite Coatings Prepared by the Pulsed Gas Dynamic Spraying Process: Effect of the Feedstock Powders', Surface and Coatings Technology, 202 (2008), 3866-77.

[86] K. Ishida, and T. Nishizawa, 'The Co-Cr (Cobalt-Chromium) System', Bulletin of Alloy Phase Diagrams, 11 (1990), 357-70.

[87] S. Hong, Y. Wu, Y. Zheng, B. Wang, W. Gao, and J. Lin, 'Microstructure and Electrochemical Properties of Nanostructured WC-10Co-4Cr Coating Prepared by HVOF Spraying', Surface and Coatings Technology, 235 (2013), 582-88.

[88] D. Mari, B. Clausen, M. A. M. Bourke, and K. Buss, 'Measurement of Residual Thermal Stress in WC-Co by Neutron Diffraction', International Journal of Refractory Metals and Hard Materials, 27 (2009), 282-87.

[89] X. J. Liu, R. Kainuma, I. Ohnuma, K. Ishida, and K. Oikawa, 'The Use of Phase Diagrams and Thermodynamic Databases for Electronic Materials', JOM, 55 (2003), 53-59.

[90] G. J. Dickins, A. M. B. Douglas, and W. H. Taylor, 'The Crystal Structure of the Co-Cr $\sigma$ Phase', Acta Crystallographica, 9 (1956), 297-303.

[91] J. D. Bolton, and M. Redington, 'Plastic Deformation Mechanisms in Tungsten Carbide', Journal of Materials Science, 15 (1980), 3150-56.

[92] V. Jayaram, 'Plastic Incompatibility and Crack Nucleation during Deformation on Four Independent Slip Systems in Tungsten Carbide-Cobalt', Acta Metallurgica, 35 (1987), 1307-15.

[93] S. B. Luyckx, 'Slip System of Tungsten Carbide Crystals at Room Temperature', Acta Metallurgica, 18 (1970), 233-36.

[94] M. K. Hibbs, and R. Sinclair, 'Room-Temperature Deformation Mechanisms and the Defect Structure of Tungsten Carbide', Acta Metallurgica, 29 (1981), 1645-54.

[95] M. Bl’anda, A. Duszová, T. Csanádi, P. Hvizdoš, F. Lofaj, and J. Dusza, 'Indentation Fatigue of WC Grains in WC-Co Composite', Journal of the European Ceramic Society, 34 (2014), 3407-12.

[96] A. Karimpoor, 'High Strength Nanocrystalline Cobalt with High Tensile Ductility', Scripta Materialia, 49 (2003), 651-56. 
[97] R. Bauer, E. A. Jägle, W. Baumann, and E. J. Mittemeijer, 'Kinetics of the Allotropic hcp-fcc Phase Transformation in Cobalt', Philosophical Magazine, 91 (2011), 437-57.

[98] H. Wei, and Y. Wei, 'Interaction between a Screw Dislocation and Stacking Faults in fcc Metals', Materials Science and Engineering: A, 541 (2012), 38-44.

[99] M. M. Myshlyaev, 'Intersection of Screw Dislocations in fcc Crystals During Torsional Deformation', Doklady Physics, 57 (2012), 107-09.

[100] W. A. Jesser, and J. W. Matthews, 'Growth of F.C.C. Cobalt on Nickel', Acta Metallurgica, 16 (1968), 1307-11.

[101] K. Chan, 'Alloying Effects on Fracture Mechanisms in Nb-Based Intermetallic in-Situ Composites', Materials Science and Engineering: A, 329-331 (2002), 513-22.

[102] P. J. Burnett, and D. S. Rickerby, 'The Mechanical Properties of Wear-Resistant Coatings: I: Modelling of Hardness Behaviour', Thin Solid Films, 148 (1987), 41-50.

[103] N. V. Demidova, X. J. Wu, and R. Liu, 'A Fracture Toughness Model for Brittle Coating on Ductile Substrate under Indentation Loading', Engineering Fracture Mechanics, 82 (2012), 17-28.

[104] W. D. Nix, and G. Huajian, 'Indentation Size Effects in Crystalline Materials: A Law for Strain Gradient Plasticity', Journal of the Mechanics and Physics of Solids, 46, 411-25.

[105] K. Chan, and D. L. Davidson, 'Delineating Brittle-Phase Embrittlement and Ductile-Phase Toughening in Nb-Based in-Situ Composites', Metallurgical and Materials Transactions A, 32, 2717-27.

[106] A. F. Bower, and M. Ortiz, 'A Three-Dimensional Analysis of Crack Trapping and Bridging by Tough Particles', Journal of the Mechanics and Physics of Solids, 39 (1991), 815-58.

[107] J. R. Pickens, and J. Gurland, 'The Fracture Toughness of WC-Co Alloys Measured on Single-Edge Notched Beam Specimens Precracked by Electron Discharge Machining', Materials Science and Engineering, 33 (1978), 135-42.

[108] S. Lay, J-L. Chermant, J. Vicens, 'Plasticity of WC Materials by T.E.M. Investigations'. In R. Tressler \& R. Bradt (Eds.), Deformation of Ceramic Materials II, Springer US, Vol.18, 1984, pp. 87-96. 
[109] Winston O. Soboyejo, T.S. Srivatsan, Advanced Structural Materials: Properties, Design Optimization, and Applications, ISBN: 978-1-57444-634-0, CRC press, 2006, pp. 189.

[110] H. W. Hugosson, O. Eriksson, U. Jansson, A. V. Ruban, P. Souvatzis, and I. A. Abrikosov, 'Surface Energies and Work Functions of the Transition Metal Carbides', Surface Science, 557 (2004), 243-54.

[111] F. J. Himpsel, J. E. Ortega, G. J. Mankey, and R. F. Willis, 'Magnetic Nanostructures', Advances in Physics, 47 (1998), 511-97.

[112] M. Alde'n, S. Mirbt, H. L. Skriver, N. M. Rosengaard, and B. Johansson, 'Surface Magnetism in Iron, Cobalt, and Nickel', Physical Review B, 46 (1992), 6303-12.

[113] D. J. Siegel, L. G. Hector Jr, and J. B. Adams, 'Adhesion, Stability, and Bonding at Metal/Metal-Carbide Interfaces: A1/WC', Surface Science, 498, 321-36.

[114] A. A. Gnidenko, 'First Principle Simulation of the Co Layers Behavior on a Surface of Hexagonal Tungsten Carbide', Physics Procedia, 23 (2012), 132-35.

[115] F. L. Zhang, C. Y. Wang, and M. Zhu, 'Nanostructured WC/Co Composite Powder Prepared by High Energy Ball Milling', Scripta Materialia, 49 (2003), 1123-28.

[116] T. Ungár, A. Borbély, G. R. Goren-Muginstein, S. Berger, and A. R. Rosen, 'Particle-Size, Size Distribution and Dislocations in Nanocrystalline Tungsten-Carbide', Nanostructured Materials, 11 (1999), 103-13.

[117] A. J. E. Foreman, 'Dislocation Energies in Anisotropic Crystals', Acta Metallurgica, 3 (1955), 322-30.

[118] S. R. Lampman, N. D. DiMatteo, and ASM International. Handbook Committee, Fatigue and Fracture (ASM International, 1996), p957.

[119] E. López Cantera, and B. G. Mellor, 'Fracture Toughness and Crack Morphologies in Eroded WC-Co-Cr Thermally Sprayed Coatings', Materials Letters, 37 (1998), 201-10.

[120] R. J. K. Wood, 'Tribology of Thermal Sprayed WC-Co Coatings', International Journal of Refractory Metals and Hard Materials, 28 (2010), 82-94.

[121] P. Chivavibul, M. Watanabe, S. Kuroda, J. Kawakita, M. Komatsu, K. Sato, and J. Kitamura, 'Effect of Powder Characteristics on Properties of Warm-Sprayed WC-Co Coatings', Journal of Thermal Spray Technology, 19 (2009), 81-88. 
[122] ASM International. Handbook Committee, Properties and Selection: Nonferrous Alloys and Special- Purpose Materials (ASM International, 1990), p2650.

[123] T. R. Duncan, 'New Data on the Shear Stresses and Energies of $1 / 2\langle 110\rangle,\{111\}$ Edge Dislocations in Elastically Anisotropic fcc Metals', Journal of Applied Physics, 39 (1968), 173.

[124] R. V. Marrey, R. Burgermeister, R. B. Grishaber, and R. O. Ritchie, 'Fatigue and Life Prediction for Cobalt-Chromium Stents: A Fracture Mechanics Analysis', Biomaterials, 27 (2006), 1988-2000.

[125] T. L. Anderson, Fracture Mechanics: Fundamentals and Applications, ISBN: 978-1-4200-5821-5, CRC Press, 1994, pp. 120.

[126] M. J. Murray, 'Fracture of WC-Co Alloys: An Example of Spatially Constrained Crack Tip Opening Displacement', Proceedings of the Royal Society of London A: Mathematical, Physical and Engineering Sciences, 356 (1977), 483-508.

[127] V. A. Ivensen, O. N. Éiduk, and V. A. Chistyakova, 'Dependence of the Yield Strength of WC-Co Hard Alloys on Their Cobalt Content and Tungsten Carbide Grain Size', Soviet Powder Metallurgy and Metal Ceramics, 13 (1974), 413-15.

[128] A. Moradkhani, H. Baharvandi, M. Tajdari, H. Latifi, and J. Martikainen, 'Determination of Fracture Toughness Using the Area of Micro-Crack Tracks Left in Brittle Materials by Vickers Indentation Test', Journal of Advanced Ceramics, 2 (2013), 87-102.

[129] Johannes Weertman, Dislocation Based Fracture Mechanics, ISBN: 978-981-02-2620-6, World Scientific Publishing Co. Pte. Ltd, 1996, pp. 206.

[130] J. L. Beuth, and N. W. Klingbeil, 'Cracking of Thin Films Bonded to Elastic-Plastic Substrates', Journal of the Mechanics and Physics of Solids, 44 (1996), 1411-28.

[131] http://www.efunda.com/materials/alloys/carbon_steels/show_carbon.cfm?ID=AISI_10 $18 \&$ show_prop=all\&Page_Title=AISI $\% 201018$

[132] S. Liu and J. A. Nairn, Fracture Mechanics Analysis of Composite Microcracking: Experimental Results in Fatigue. Proc. of the Amer. Soc. of Comp., 5th Tech. Conf. (1990) 287-295.

[133] M. M. Nagl, and W. T. Evans, 'The Mechanical Failure of Oxide Scales under Tensile or Compressive Load', Journal of Materials Science, 28 (1993), 6247-60. 
[134] S. S. Chiang, D. B. Marshall, and A. G. Evans, 'The Response of Solids to Elastic/Plastic Indentation. I. Stresses and Residual Stresses', Journal of Applied Physics, 53 (1982), 298.

[135] H. Ichimura, F.M. Rodriguez, and A. Rodrigo, 'The Composite and Film Hardness of TiN Coatings Prepared by Cathodic Arc Evaporation', Surf. Coat. Technol. 127 (2000) 138.

[136] J. Malzbender, G. de With, and J. M. J. den Toonder, 'Elastic Modulus, Indentation Pressure and Fracture Toughness of Hybrid Coatings on Glass', Thin Solid Films, 366 (2000), $139-49$.

[137] S. Smith, and R. Scattergood, 'Crack-shape Effects for Indentation Fracture Toughness Measurements', J. Am. Ceram. Soc., 75 [2] 305-15. 


\section{Appendix}

The main program of the crystal structure models:

$\% \% \mathrm{WC}$

$\mathrm{k}=1.38 \mathrm{e}-23$;

$\mathrm{Ewc}=668 \mathrm{e} 9$

$\mathrm{vwc}=0.24$

$\mathrm{bwc}=0.2906 \mathrm{e}-9$;

$p w c=0.2517 \mathrm{e}-9$;

uslipwc=269e9;

$1=308.3 e-6$

$c=497.7 \mathrm{e}-6$;

$\mathrm{f}=4.1162$;

$\mathrm{t}=45 \mathrm{e}-6$;

theta $=0.3832$;

phwc $=2 * t * 1.5 * \tan ($ theta $) /\left(b_{w c} * f^{\wedge} 2\right)$

yuswc $=\left(\right.$ uslipwc $\left.{ }^{*} \mathrm{bwc}^{\wedge} 2\right) /\left(2 * \mathrm{pi}^{\wedge} 2 * \mathrm{pwc}\right)$

kcfitwc $=1.2369$

phiwc $=($ pi*pwc*kcfitwc $) / b w c$

Upnwc $=\left(8 * \mathrm{pi}^{\wedge} 2 *\right.$ yuswc $) / \sinh (2 *$ phiwc $)$ 
$\mathrm{Jswc}=5.28$

$\mathrm{yfwc}=0.05$;

$\mathrm{T}=1$;

$\mathrm{J} 0 \mathrm{wc}(1)=\left(\mathrm{k}^{*} \mathrm{~T} /(\mathrm{bwc})^{\wedge} 2\right)^{*} \mathrm{phwc}$

$\operatorname{EEEwc}(1)=\exp \left(-b_{w c} \wedge 2 *(U p n w c+y f w c) /\left(k^{*} \mathrm{~T}\right)\right)$

$\operatorname{Jdwc}(1)=\mathrm{J} 0 \mathrm{wc}(1) * \exp \left(-\mathrm{bwc}^{\wedge} 2 *(\mathrm{Upnwc}+\mathrm{yfwc}) /\left(\mathrm{k}^{*} \mathrm{~T}\right)\right)$

$\operatorname{Kcwc}(1)=\left(\operatorname{Ewc} *\left(\operatorname{Jdwc}(1)+\mathrm{JSwc}_{\mathrm{Sw}}\right) /\left(1-\mathrm{vwc}^{\wedge} 2\right)\right)^{\wedge}(0.5)$

$\% \% \mathrm{Co}$

$\mathrm{k}=1.38 \mathrm{e}-23$;

$\mathrm{Eco}=209 \mathrm{e}$;

$\mathrm{vco}=0.31$

$\mathrm{bco}=0.2519 \mathrm{e}-9$

pco $=0.2057 \mathrm{e}-9$

uslipco=75e9;

phco $=2 * t^{*} 1.5 * \tan ($ theta $) /\left(\right.$ bco $\left.^{*} \mathrm{f}^{\wedge} 2\right)$

yusco $=\left(\right.$ uslipco $\left.* \mathrm{bco}^{\wedge} 2\right) /\left(2 * \mathrm{pi}^{\wedge} 2 * \mathrm{pco}\right)$

kcfitco $=1.3$

phico $=($ pi*pco*kcfitco $) / b c o$;

Upnco $=\left(8 * \mathrm{pi}^{\wedge} 2 *\right.$ yusco $) / \sinh (2 *$ phico $)$ 
$\mathrm{Jsco}=5.4$

$\mathrm{yfco}=0.0135$

$\mathrm{T}=1$;

$\mathrm{J} 0 \operatorname{co}(1)=\left(\mathrm{k}^{*} \mathrm{~T} /(\mathrm{bco})^{\wedge} 2\right)^{*}$ phco;

$\operatorname{EEEco}(1)=\exp \left(-\mathrm{bco}^{\wedge} 2 *(\right.$ Upnco + yfco $\left.) /\left(\mathrm{k}^{*} \mathrm{~T}\right)\right)$

$\mathrm{Jdco}(1)=\mathrm{J} 0 \operatorname{co}(1) * \exp \left(-\mathrm{bco}^{\wedge} 2 *(\mathrm{Upnco}+\mathrm{yfco}) /\left(\mathrm{k}^{*} \mathrm{~T}\right)\right)$

$\operatorname{Kcco}(1)=\left(\operatorname{Eco} *(\operatorname{Jdco}(1)+\mathrm{Jsco}) /\left(1-\mathrm{vco}^{\wedge} 2\right)\right)^{\wedge}(0.5) ;$

$\mathrm{Vh}=0.7642$

$\mathrm{Z}=2 /(\mathrm{pi})^{\wedge}(1 / 2)$

$\operatorname{Kcc}(1)=\operatorname{Kcwc}(1)^{*}\left(1+Z^{*}(1-\mathrm{Vh})^{\wedge}(0.5)^{*}\left((\operatorname{Kcco}(1) / \operatorname{Kcwc}(1))^{\wedge} 2-1\right)\right)^{\wedge}(0.5)$

$\mathrm{n}=1$;

for $\mathrm{i}=1: 1000$

$\mathrm{T}=1+\mathrm{i}-1$

$\mathrm{J} 0 \mathrm{wc}(\mathrm{n})=\left(\mathrm{k}^{*} \mathrm{~T} /(\mathrm{bwc})^{\wedge} 2\right)^{*} \mathrm{phwc}$

$\operatorname{EEEwc}(\mathrm{n})=\exp \left(-\mathrm{bwc}^{\wedge} 2 *(\mathrm{Upnwc}+\mathrm{yfwc}) /\left(\mathrm{k}^{*} \mathrm{~T}\right)\right)$

$\mathrm{Jdwc}(\mathrm{n})=\mathrm{J} 0 \mathrm{wc}(\mathrm{n}) * \exp \left(-\mathrm{bwc}^{\wedge} 2 *(\mathrm{Upnwc}+\mathrm{yfwc}) /\left(\mathrm{k}^{*} \mathrm{~T}\right)\right)$

$\operatorname{Kcwc}(\mathrm{n})=\left(\operatorname{Ewc} *\left(\operatorname{Jdwc}(\mathrm{n})+\mathrm{JSWc}_{\mathrm{Sw}}\right) /\left(1-\mathrm{vwc}^{\wedge} 2\right)\right)^{\wedge}(0.5)$

$\mathrm{J} 0 \operatorname{co}(\mathrm{n})=\left(\mathrm{k}^{*} \mathrm{~T} /(\mathrm{bco})^{\wedge} 2\right)^{*} \mathrm{phco}$ 
$\operatorname{EEEco}(\mathrm{n})=\exp \left(-\mathrm{bco}^{\wedge} 2 *\left(\mathrm{Upnco}^{+}+\mathrm{yfco}\right) /\left(\mathrm{k}^{*} \mathrm{~T}\right)\right)$

$\mathrm{Jdco}(\mathrm{n})=\mathrm{J} 0 \operatorname{co}(\mathrm{n}) * \exp \left(-\mathrm{bco}^{\wedge} 2^{*}(\mathrm{Upnco}+\mathrm{yfco}) /\left(\mathrm{k}^{*} \mathrm{~T}\right)\right) ;$

$\operatorname{Kcco}(\mathrm{n})=\left(\operatorname{Eco} *\left(\operatorname{Jdco}(\mathrm{n})+\mathrm{JSco}_{\mathrm{Sco}}\right) /\left(1-\mathrm{vco}^{\wedge} 2\right)\right)^{\wedge}(0.5)$

$\operatorname{Kcc}(n)=\operatorname{Kcwc}(n) *\left(1+Z^{*}(1-V h)^{\wedge}(0.5)^{*}\left((\operatorname{Kcco}(n) / \operatorname{Kcwc}(n))^{\wedge} 2-1\right)\right)^{\wedge}(0.5) ;$

$\mathrm{n}=\mathrm{n}+1$

end

figure(1)

$\operatorname{plot}([1: 1: 1000], \mathrm{Kcwc})$;

title('WC Fracture toughness')

figure(2)

$\operatorname{plot}([1: 1: 1000], \mathrm{Kcco})$

title('Co Fracture toughness')

figure(3)

$\operatorname{plot}([1: 1: 1000], \mathrm{Kcc})$

title('WC-10Co4Cr Fracture toughness')

figure(4)

$\operatorname{plot}([1: 1: 1000]$, EEEwc); 
title('expwc')

figure(5)

$\operatorname{plot}([1: 1: 1000]$, EEEco);

title('expco')

temp $=[1: 1: 1000] ;$

$\mathrm{wc}(:, 1)=$ temp;

$\mathrm{wc}(:, 2)=\mathrm{Kcwc}$

$\operatorname{co}(:, 1)=$ temp;

$\operatorname{co}(:, 2)=\mathrm{Kcco}$;

$\operatorname{wcco}(:, 1)=$ temp;

$\operatorname{wcco}(:, 2)=\mathrm{Kcc}$;

fid=fopen('wc.txt','wt');

$[\mathrm{m}, \mathrm{n}]=\operatorname{size}(\mathrm{wc})$;

for $\mathrm{i}=1: 1: \mathrm{m}$

for $\mathrm{j}=1: 1: \mathrm{n}$

if $j==n$

fprintf(fid, '\%g $\%$ 'n',wc(i,j));

else

fprintf(fid, $\left.{ }^{\prime} \% \mathrm{~g} \backslash \mathrm{t}^{\prime}, \mathrm{wc}(\mathrm{i}, \mathrm{j})\right)$; 
end

end

end

fclose(fid);

fid=fopen('co.txt','wt');

$[\mathrm{m}, \mathrm{n}]=\operatorname{size}(\mathrm{co})$

for $\mathrm{i}=1: 1: \mathrm{m}$

for $\mathrm{j}=1: 1: \mathrm{n}$

if $j==n$

fprintf(fid, $\left.{ }^{\prime} \% g \backslash n ', \operatorname{co}(i, j)\right)$;

else

fprintf(fid, $\left.{ }^{\prime} \% \mathrm{~g} \backslash \mathrm{t}^{\prime}, \mathrm{co}(\mathrm{i}, \mathrm{j})\right)$;

end

end

end

fclose(fid);

fid=fopen('wcco.txt','wt');

$[\mathrm{m}, \mathrm{n}]=\operatorname{size}(\mathrm{wcco})$

for $\mathrm{i}=1: 1: \mathrm{m}$ 
for $\mathrm{j}=1: 1: \mathrm{n}$

if $j==n$

fprintf(fid, '\%g $\%$ n',wcco(i,j));

else

fprintf(fid, $\left.\% \mathrm{~g} \backslash \mathrm{t}^{\prime}, \mathrm{wcco}(\mathrm{i}, \mathrm{j})\right)$;

end

end

end

fclose(fid);

The main program of the microcrack formation models:

$\mathrm{k}=1.38 \mathrm{e}-23$;

$\mathrm{E}=310 \mathrm{e}$;

$\mathrm{v}=0.3$;

$1=368.75 \mathrm{e}-6$;

$c=497.7 e-6$;

$\mathrm{f}=4.1162$;

$\mathrm{h}=\mathrm{l}^{*} \mathrm{f}$

theta $=0.3832$; 
phi $=1.1868$;

$\mathrm{t}=45 \mathrm{e}-6$;

$F=0.64$;

$\mathrm{Y}=380 \mathrm{e} 6$

sigma $=200 \mathrm{e} 6$

$\mathrm{Kic}=2.78 \mathrm{e} 6$;

$\mathrm{P}=196$

$\mathrm{pp}=\mathrm{P} /\left(2 * 1^{\wedge} 2\right)$

tension $=\mathrm{pp}^{*} \cos (1.1868)$

sigmars $=\mathrm{pp}^{*}\left((3 *(\log (\mathrm{f})+0.5) /(1+3 * \log (\mathrm{f})))-1-1 /\left(2 * \mathrm{f}^{\wedge} 3\right)\right)$

$\mathrm{T}=1$;

$\mathrm{p}=\left(\mathrm{Y}^{\wedge} 2\right) /\left(3 * 4 * \mathrm{t}^{\wedge} 2 * \operatorname{sigma}^{\wedge} 2\right)$

space $=1.732 * 2 *$ sigmars $* t / Y$

$\mathrm{D}=1 /$ space;

$\mathrm{Ge}=\left(\operatorname{sigma}{ }^{\wedge} * \mathrm{t} / \mathrm{E}\right) *\left(\mathrm{pi}^{*} \mathrm{~F}+(\operatorname{sigma} /(1.732 * \mathrm{Y}))\right)$ 
$\mathrm{rho}=\mathrm{Ge} /(2 * 210 \mathrm{e} 6)$

$Y_{S}=2.47$

$\mathrm{a}=118.468$

$b=81$;

$\operatorname{Gss}(1)=\left(\mathrm{k}^{*} \mathrm{~T} /(\mathrm{rho} /(\mathrm{a}))^{\wedge} 2\right)^{*} \mathrm{a} * \mathrm{D} * 2 * \mathrm{c} * \exp \left(-\mathrm{Ge}^{*}(1 / \mathrm{b}) *(\mathrm{rho} /(\mathrm{a}))^{\wedge} 2 /\left(\mathrm{k}^{*} \mathrm{~T}\right)\right)$

$\operatorname{EEE}(1)=\exp \left(-\mathrm{Ge}^{*}(1 /(\mathrm{a}))^{*}(\mathrm{rho} / \mathrm{a})^{\wedge} 2 /\left(\mathrm{k}^{*} \mathrm{~T}\right)\right)$

$\operatorname{Kss}(1)=\left(\left((\operatorname{Gss}(1))^{*} \mathrm{E}\right) /\left(1-\mathrm{v}^{\wedge} 2\right)\right)^{\wedge}(1 / 2)$

$\mathrm{n}=1$;

for $\mathrm{i}=1: 1000$

$\mathrm{T}=1+\mathrm{i}-1$

$\operatorname{Gss}(\mathrm{n})=\left(\mathrm{k}^{*} \mathrm{~T} /(\mathrm{rho} /(\mathrm{a}))^{\wedge} 2\right)^{*} \mathrm{a}^{*} \mathrm{D} * 2 * \mathrm{c}^{*} \exp \left(-\mathrm{Ge} *(1 / \mathrm{b})^{*}(\mathrm{rho} /(\mathrm{a}))^{\wedge} 2 /\left(\mathrm{k}^{*} \mathrm{~T}\right)\right)$

$\operatorname{EEE}(\mathrm{n})=\exp \left(-\mathrm{Ge}^{*}(1 /(\mathrm{a})) *(\mathrm{rho} / \mathrm{a})^{\wedge} 2 /\left(\mathrm{k}^{*} \mathrm{~T}\right)\right)$

$\operatorname{Kss}(n)=\left(\left((\operatorname{Gss}(n))^{*} \mathrm{E}\right) /\left(1-\mathrm{v}^{\wedge} 2\right)\right)^{\wedge}(1 / 2)$ 
$\mathrm{n}=\mathrm{n}+1$;

end

figure(1)

$\operatorname{plot}([1: 1: 1000], G s s)$;

title('energy release rate')

figure(2)

$\operatorname{plot}([1: 1: 1000], \mathrm{Kss})$;

title('Fracture toughness')

figure(3)

$\operatorname{plot}([1: 1: 1000], \mathrm{EEE})$;

title('exp')

$\mathrm{Kcc}=(\mathrm{Ge} * \mathrm{E} /(1-\mathrm{v}))^{\wedge} 0.5$

temp $=[1: 1: 1000]$;

$\mathrm{ft}(:, 1)=$ temp;

$\mathrm{ft}(:, 2)=\mathrm{Kss} ;$

fid=fopen('1962.txt','wt');

$[\mathrm{m}, \mathrm{n}]=\operatorname{size}(\mathrm{ft})$; 
for $\mathrm{i}=1: 1: \mathrm{m}$

for $\mathrm{j}=1: 1: \mathrm{n}$

if $j==n$

fprintf(fid, $\left.{ }^{\prime} \% \mathrm{~g} \backslash \mathrm{n}^{\prime}, \mathrm{ft}(\mathrm{i}, \mathrm{j})\right)$;

else

fprintf(fid, '\%g $\left.\% t^{\prime}, f t(i, j)\right)$;

end

end

end

fclose(fid); 